SINK ELECTRICAL DISCHARGE MACHINING OF SUPERHYDROPHOBIC SURFACES 


\title{
SINK ELECTRICAL DISCHARGE MACHINING OF SUPERHYDROPHOBIC SURFACES
}

\author{
BY \\ CHANGCHENG GUO, B. ENG
}

\author{
A Thesis \\ Submitted to the School of Graduate Studies in Partial Fulfilment \\ of the Requirements for the Degree of \\ Master of Applied Science
}

McMaster University (C) Copyright by Changcheng Guo, August 2018 
Master of Applied Science (2018)

(Department of Mechanical Engineering)

Title:

Author:

Changcheng Guo

B. ENG (Mechanical Engineering)

McMaster University, Hamilton, Ontario

Supervisors:

Dr. Philip Koshy

Dr. P. Ravi Selvaganapathy

Number of Pages: $\quad$ xiv, 91

of Superhydrophobic Surfaces
McMaster University

Hamilton, Ontario, Canada 


\section{Abstract}

Water-repellent behaviour, known as hydrophobicity, has recently attracted a great deal of interest due to its applications, such as anti-icing and self-cleaning. The phenomenon of hydrophobicity found in surfaces like lotus leaves is manifest by a hierarchical structure on low-energy surfaces. Fabrication of hydrophobic surfaces has thus far been largely accomplished on polymers and colloidal materials, which are limited by poor mechanical strength that leads to performance degradation over time. To this end, fabrication of a robust metallic hydrophobic surface is the focus of this research. Sink electrical discharge machining is demonstrated to generate hydrophobic surfaces in 7075 aluminum alloy with water contact angles in excess of $150^{\circ}$. 


\section{Acknowledgements}

I would like to thank all the people who helped me accomplish this work.

First and foremost, I would like to express my deep gratitude to my supervisor Dr. Philip Koshy. Your high standard of work molded my prudent attitude towards research. Your eagerness of science inspired my passion of learning and contributing. Your continued encouragement and support built who I became today. My graduation study could not be such enjoyable without you.

In addition, I would like to thank my co-supervisor, Dr. P. Ravi. Selvaganapathy. Your knowledge regarding microfluidics helped me overcome countless difficulties. Your open-minded engineering thinking broadened my horizons and raised the project to a higher level. Your optimism could always tackle the problems in the easiest but most affective way. I was truly glad to work with you.

Many thanks to other members in Dr. Koshy's research team. Gurpyar Dhadda and Jason Chan, thanks for your company within this two years, I enjoyed sharing knowledge, discussing topics and even arguing with you. Joseph Henry Span and Salomon C. Gabriel, thanks for all the help when I just came to the family. You guided me during my most confused period, and you trained me with all what you had. All what I got could not be accomplished without the pushes from each of you. It was my honor to meet all of you in my life. 
Also, I really appreciated all the stuff working on second floor machine shop, John Colenbrander and Michael Lee, who trained me with the machines patiently; Ron Lodewyks and Mark Mackenzie, who provided me valuable suggestions with the purchases, Joe Verhaeghe and Dan Wright, who helped me more than many times with either software problems or EDM electrical issues. Unfortunately, we lost Dan in 2018, which was a tremendous loss to his family, to university and to the society. His spirit and smile would be with us forever. I would also like to thank all the stuff working on third floor mechanical office, Lily Sazz-Fayter, Florence Rosato, Leslie Kocsis and Nicole Mclean. I always saw you as the most significant people in the department. Mechanical engineering could not keep moving without all your hard working.

Thank you to every single member working in MMRI. Terry, who mastered all the machines in the lab; Brady, who always had some ideas about your hardship; Sophia, who helped me countless times on different microscopic works; Luke, who polished my metallic surfaces like mirrors; Kan, who provided the most fabulous videos through the high speed camera; Nino, who encouraged me all the time to be a good researcher. There were also Rohan, Shafiul, Majid and so on, who treated me like a brother. Some people were not mentioned only because of the limitation of pages, which did not indicate your insignificance in my study. A special thank to Maryam Aramesh, who brought me into the world of metal removal, to MMRI, and to Dr. Koshy. I always saw you as the most knowledgeable and amiable person in the lab. I felt quite fulfilled working with all of you, and meeting all of you. 
Most importantly, I wanted to say thank you to my family, to all the relatives who had ever helped me in these years. 谢谢爸妈对我二十四年的精心栽培; 谢谢老一辈们 的全力以赴, 包括一七年我生日那天逝世的姥爷; 谢谢郭家家和冯家家所有父辈的鼎 力相助; 谢谢所有兄弟姐妹的默默支持, 特别是姐姐的无微不至; 谢谢我的朋友们, 尤其是我的女朋友, 在我手术住院期间，和那个伤心的夏天里无时无刻的陪伴; 最 后也感谢所有亲戚朋友的帮助与厚爱, 没有大家, 便没有长城的今天。永远爱你们! 


\section{Contents}

Abstract

Acknowledgements $\quad$ iv

1 Introduction $\quad 1$

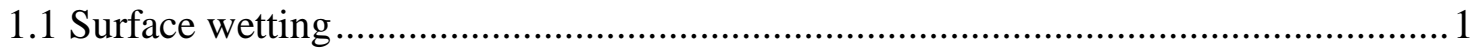

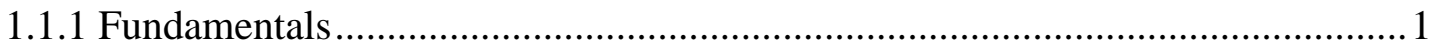

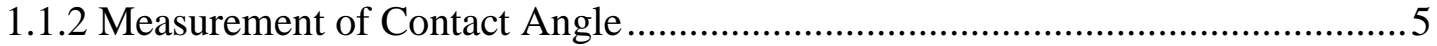

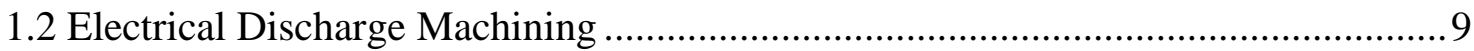

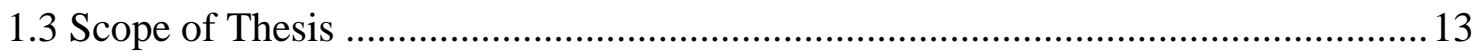

2 Literature Review $\quad 15$

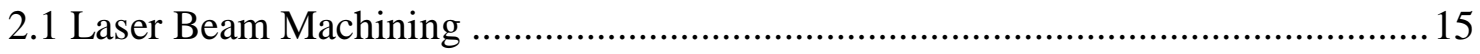

2.1.1 LBM of Metallic Superhydrophobic Surfaces ........................................ 17

2.1.2 LBM of Non-Metallic Superhydrophobic Surfaces ....................................21

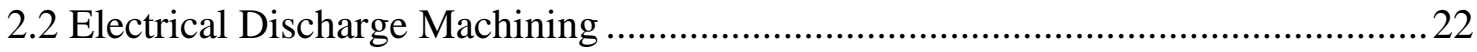

2.2.1 Wire EDM of Superhydrophobic Surfaces ...............................................23

2.2.2 Sink EDM of Superhydrophobic Surfaces ...............................................2

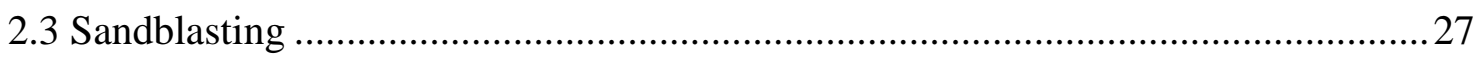

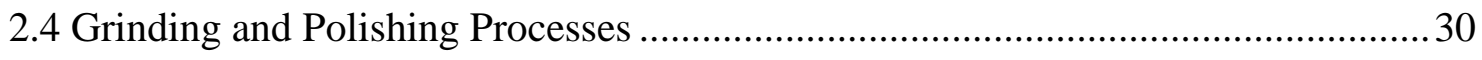

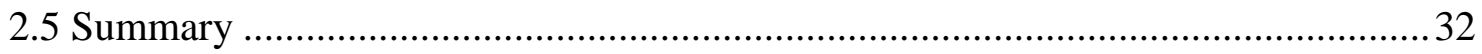




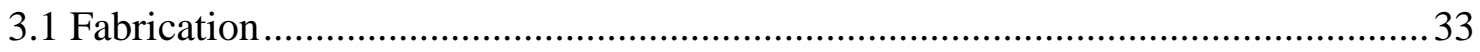

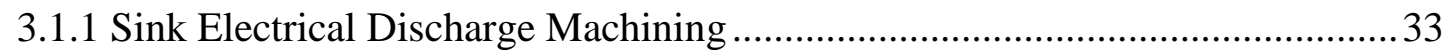

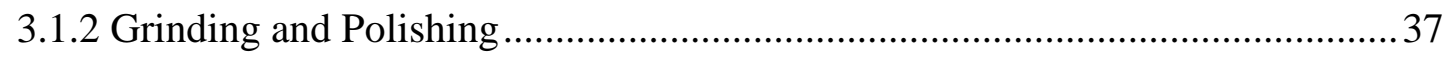

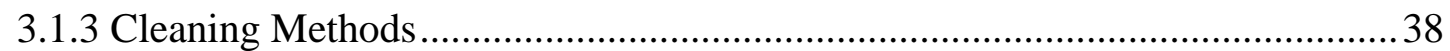

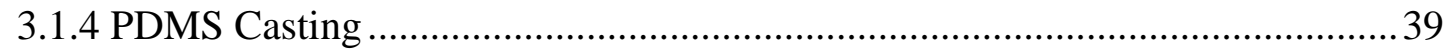

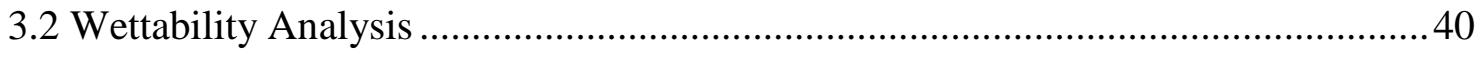

3.2.1 Contact Angle Measurement ............................................................... 41

3.2.2 Contact Angle Hysteresis Measurement...................................................... 43

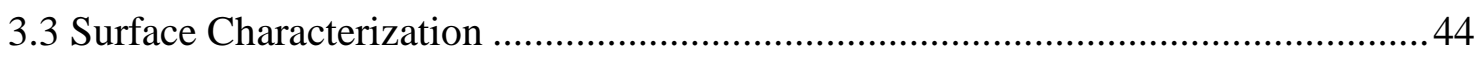

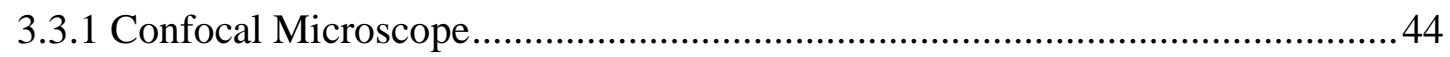

3.3.2 Scanning Electron Microscope ............................................................. 46

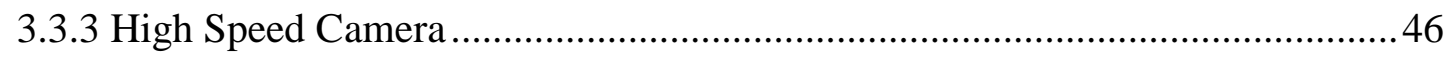

4. Results and Discussion 48

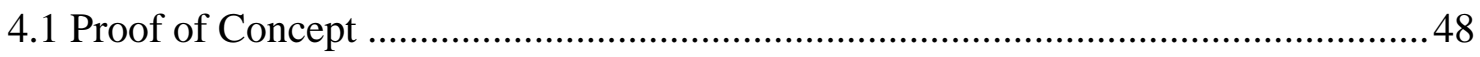

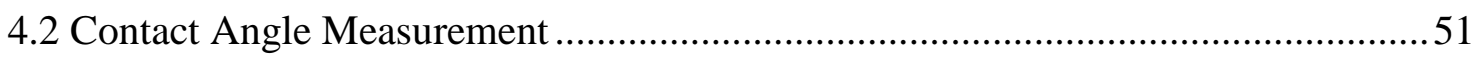

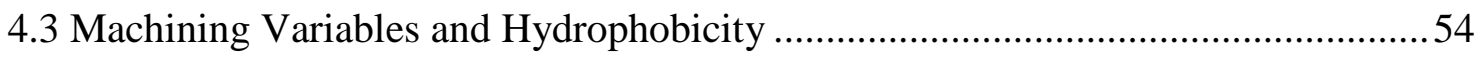

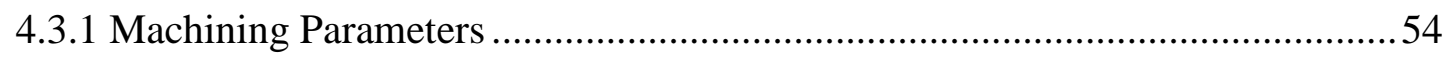

4.3.2 Surface Structural Characterization......................................................... 67

4.3.3 Workpiece Material Al 7075 and Al 6061 ..............................................69

4.3.4 Contact Angle Degradation by Using EDMed Electrode Surface ................... 71

4.3.5 Sink EDM Experiment in Water and Time Influence .................................. 73

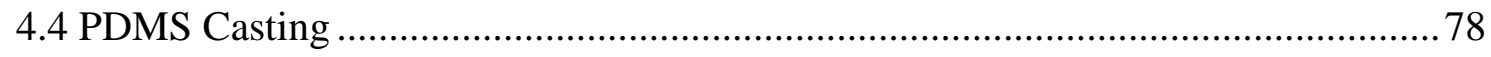


5. Conclusions and Future Work

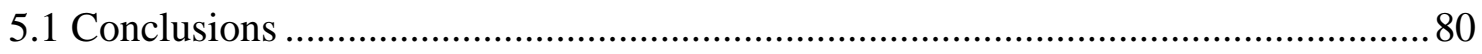

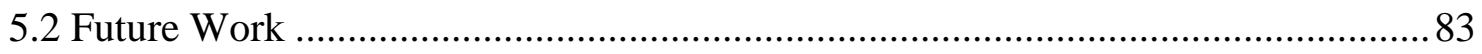

$\begin{array}{ll}\text { 6. References } & 85\end{array}$ 


\section{List of Tables}

4.1 Contact Angles Generated by Four Fitting Methods.........................................52

4.2 Chemical Composition of $\mathrm{Al} 7075$ and $\mathrm{Al} 6061$................................................... 70

4.3 Contact Angles on Casted PDMS and Relative Aluminum Alloy Surfaces ...........78 


\section{List of Figures}

1.1.1 Schematic view of Young's angle, or static contact angle ..............................2

1.1.2 Schematic view of Advancing and Receding contact angles..............................

1.1.3 Wenzel State (left) and Cassie Baxter State (right) on rough surfaces ...............4

1.1.4 a) SEM image of microscale protrusions and b) nanoscale protrusions on lotus leaves. c) A water droplet sitting on a lotus leaf. d) Schematic two-dimensional view of a water droplet sitting on the hierarchical structure ...........................

1.1.5 OCA 35 High Speed Contact Angle Measurement Machine includes (a) a highresolution video camera, (b) a horizontal stage, (c) a halogen light source, (d) a removable needle, (e) and a microliter syringe . .8

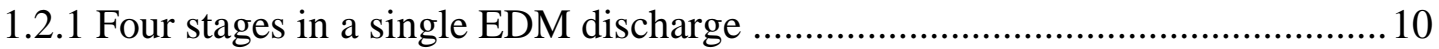

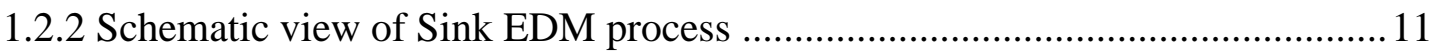

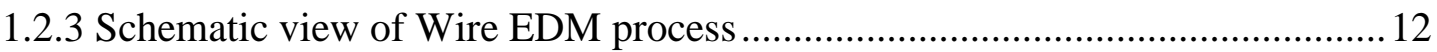

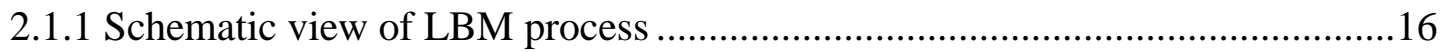

2.1.2 Contact angle change over time for six materials with a laser fluence of

$0.78 \mathrm{~J} / \mathrm{cm}^{2}$ 18

2.1.3 (left) SEM of laser machined AISI 316L Stainless Steel with fluence of $2.4 \mathrm{~J} / \mathrm{cm}^{2}$, (right) and a water drop profile on its surface with a contact angle of $166.3^{\circ} \pm 1.1^{\circ}$

2.1.4 Schematic view of the wettability transition by LBM and the post process......21

2.1.5 Performance of the laser textured sapphire surface: (a) high water repellency

(b) high transparency (c) self-cleaning effect.

2.2.1 Schematic view of Wire EDM simultaneous dual-structure fabrication process 
2.2.2 (left) Schematic illustration of superhydrophobic polymer sheets manufacturing process by using Wire EDM textured roller [28], (right) and the effect water-oil separation on two stainless steel samples with different sizes of grooves textured by using Wire EDM

2.2.3 (a) An example of the re-entrant microstructure machined by using micro Wire EDM, with (b) a water drop sitting on it and (c) a RL-68H oil sitting on it .....25

2.2.4 A schematic illustration of the 3-step Sink EDM fabrication process of superhydrophobic surfaces

2.3.1 Schematic illustration of three-step sandblasting process to fabricate superhydrophobic surfaces .28

2.3.2 Manufacturing process of polymeric superhydrophobic surfaces by using Nickel mold fabricated from sandblasting and chemical etching .29

2.4.1 Surface profile of the workpiece cut by ultrasonic elliptical vibration cutting (a) in the feed direction and (b) the cutting direction ...............................................31

2.4.2 An example of observing contact angle from different directions .31

3.1.1 The AGIETRON Impact 2 Sink EDM. .34

3.1.2 Machining setup with the copper electrode clamped in the quill, and the $\mathrm{Al}$ 7075 workpiece clamped in the vice

3.1.3 Struers Tegramin-25 Polisher (left) and polished specimens (right) ...................37

3.1.4 An EDM textured surface with its PDMS casted surface ....................................40

3.2.1 Process of dispensing the droplet onto the textured surface

3.2.2 Needle embedded dragging method to measure CAH moving with a speed of $0.13 \mathrm{~mm} / \mathrm{s}$

3.3.1 Alicona InfiniteFocus G5 White Light Interferometer .45

4.1.1 Water droplets on a polished surface and a Sink EDM textured surface taken by $(a, c)$ regular microscopy and $(b, d)$ goniometer respectively 
4.1.2 Picture sequences of an impacting water droplet

4.1.3 Picture sequence of an impacting water drop on an EDM textured surface tilted at $15^{\circ}$ .50

4.2.1 Manual contact angle measurements on three surfaces .51

4.2.2 Four fitting methods: (a) Ellipse fitting, (b) Circle fitting, (c) Tangential fitting, and (d) Y-L fitting use to calculate the static contact angle of a sessile droplet

4.3.1 Contact angle Vs. Current in 3 groups of discharge durations values with (a), (b), (c) primary texturing only and (d), (e), (f) primary texturing plus secondary texturing......

4.3.2 Fabrication of pillar array designs with a) circular, b) triangular, c) square, and

d) cross-shape top area to investigate the hydrophobicity .59

4.3.3 Contact angle Vs. pillar spacing-to-width ratio with the analysis of Cassie Baxter State and Wenzel State

4.3.4 Contact angle Vs. Secondary machining time with different groups of primary texturing parameters .63

4.3.5 Contact angle Vs. discharge duration with a current of 6.2 A..... .65

4.3.6 Aluminum alloy surfaces, machined with small parameters, generated black depositions

4.3.7 Profiles and histograms of the surfaces generated by (a) primary texturing only and (b) primary + secondary texturing with the distribution histogram on the side.

4.3.8 Fast Fourier Transform of the surface profiles shown in Fig. 4.3.7

4.3.9 Contact angle Vs. Secondary machining time for Al 7075 and Al 6061 alloys 
4.3.10 Contact angle Vs. Number of machining times of used electrode surfaces

4.3.11 Contact angle Vs. Curing time for three groups of machining: (a) machined in hydrocarbon oil, (b) machined in DI water, and (c) machined in DI water, then

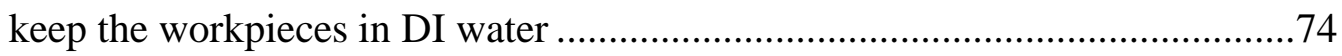

4.3.12 Profiles of the surfaces machined in water and oil .................................... 75

4.3.13 Fast Fourier Transform of the surfaces shown in Fig. 4.3.12 ........................76

4.3.14 SEM and EDM analysis for three surfaces: (1) one day after machined in DI water, (2) one day after machined in hydrocarbon oil, and (3) 15 days after

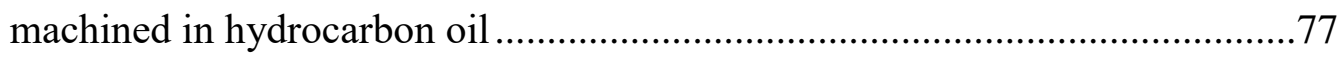

5.2.1 Surface chemistry analysis by using EDS for the surfaces machined after (left) 1

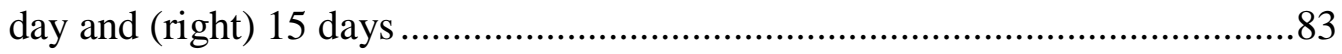




\section{Chapter 1}

\section{Introduction}

In the recent decades, learning from lotus leaves (Nelumbos), superhydrophobic surfaces have drawn a great amount of interest. They can be widely used in a variety of fields, like medical devices due to their non-wetting property; solar panels due to anti-icing effect; satellite dishes due to self-cleaning phenomenon and so on. Most of the materials being utilized are colloidal materials or polymers which can be easily worn out over time; therefore, metallic superhydrophobic surfaces are always desired. This chapter explains the principles of wettability, and the motivation behind generating a robust superhydrophobic surface. It also introduces the fundamentals of a goniometer used for surface wettability characterization, as well as the Sink Electrical Discharge Machining which is the fabrication technology being used in the current work.

\subsection{Surface Wetting}

\subsubsection{Fundamentals}

A Chinese poem from a thousand years ago describes the lotus leaf as a sign of purity due to its unique property of being able to emerge from the muddy waters entirely unstained. People could not explain the reason for this phenomenon then, but only knew that lotus leaves could not be wetted. Wettability of a solid surface was first investigated by Young 
[1] in 1805. He defined the tangential angles at the liquid-solid-air interface, arising from the steady state of a mechanical equilibrium of a water droplet resting on a surface. This angle was named Young's angle or static contact angle $\left(\theta_{C}\right)$, shown in Fig. 1.1.1.

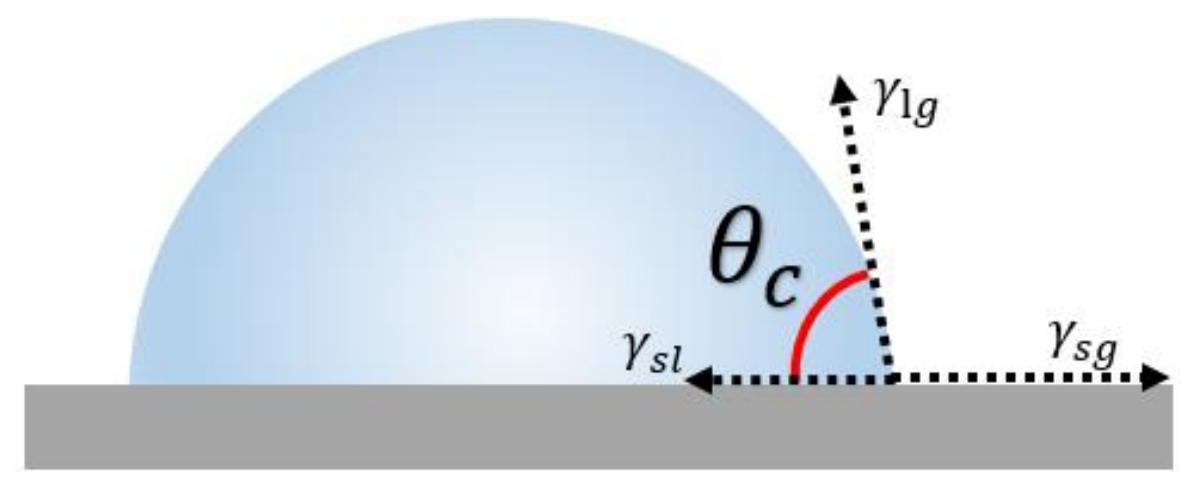

Fig. 1.1.1 Schematic view of Young's angle, or static contact angle.

The static contact angle can be computed in relation to three surface tensions

(Eq. 1.), the solid surface tension $\gamma_{s g}$, the liquid surface tension $\gamma_{l g}$, and the solid liquid interfacial tension $\gamma_{s l}$.

$$
\gamma_{s g}=\gamma_{l g} \cos \theta_{c}+\gamma_{s l}
$$

According to Law et al. [2], Rayleigh [3] and Bartell [4] first reported on another two contact angles, normally the advancing and receding contact angles (Fig. 1.1.2), when the waterdrop slides on a surface with a certain velocity. The difference between these 
angles was termed the contact angle hysteresis $(\mathrm{CAH})$ which became a significant factor in wettability research.

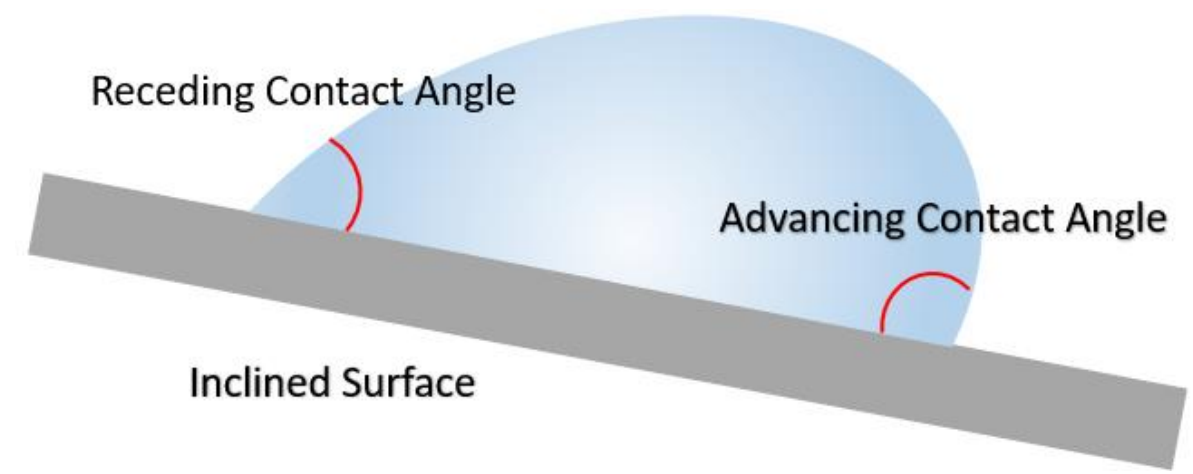

Fig. 1.1.2 Schematic view of Advancing and Receding contact angles.

In 1936, the concept of wetting states on a rough surface was first described by Wenzel [5]. He analyzed the homogeneous wetting regime, where the liquid could fully wet the surface. The apparent contact angle could be computed according to the surface roughness and the intrinsic contact angle of the material [Eq. 1.2].

$$
\cos \theta_{w}=r \cos \theta_{c}
$$

where $\theta_{w}$ is the apparent contact angle corresponding to Wenzel wetting state (Fig. 1.1.3 left), and $r$ is the roughness factor which is the ratio of real surface area to projected surface area. Another wetting regime was discovered by Cassie and Baxter in 1944 [6]. By 
observing the behaviour of water droplets resting on porous surfaces, they discovered a more complex heterogeneous wetting state, where air bubbles could be entrapped within the porous structure. Less energy is needed for the waterdrop to roll on such a surface due to the reduction of liquid-solid contact. The apparent contact angle in this case can be expressed as Eq. 3.:

$$
\cos \theta_{C B}=f \cos \theta_{c}+f-1
$$

where $\theta_{C B}$ is the apparent contact angle corresponding to Cassie Baxter wetting state (Fig. 1.1.3 right) and $f$ is the solid area fraction.
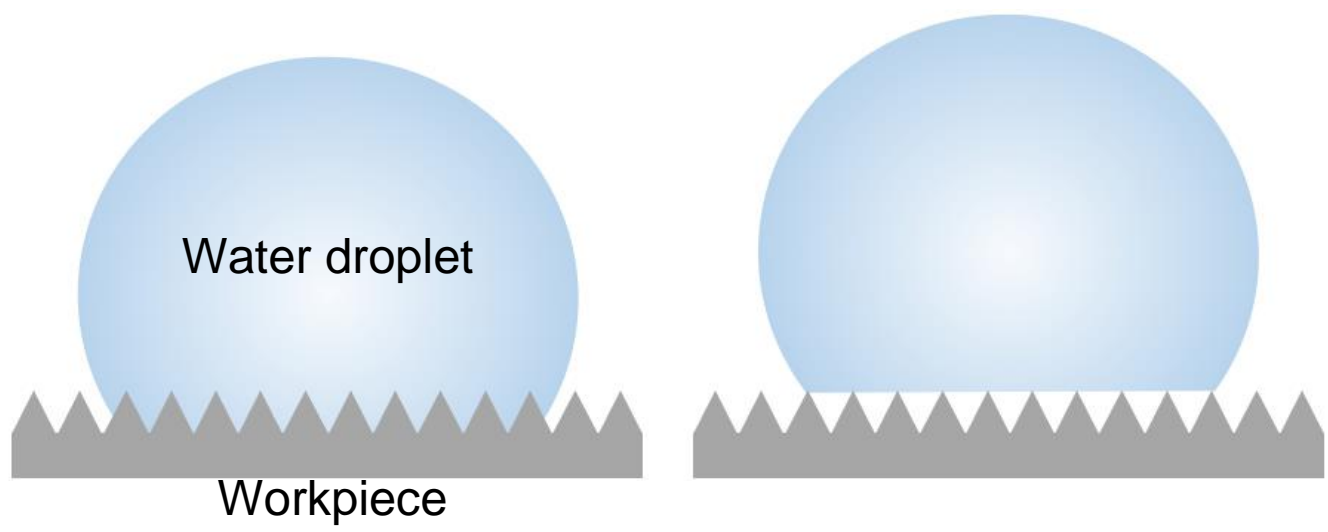

Fig. 1.1.3 Wenzel State (left) and Cassie Baxter State (right) on rough surfaces.

In 1964, superhydrophobicity was first studied by Dettre and Johnson after the introduction of scanning electron microscope (SEM) [7]. They discovered the importance 
of roughness in the context of high water repellency. Until 1977, monolithic structure of the surface and low surface energy of the material were known to form superhydrophobic surfaces. Wilhelm et al. [8] explained the significance of the secondary structure on the top of the rough surface, which formed a hierarchical structure. They found the self-cleaning behaviour of lotus leaves was due to not only their dual-structure, but also the wax layer on the top of their surfaces. This discovery referred to the critical factors for superhydrophobicity: a hierarchical structure and low surface energy. As shown in Fig. 1.1.4, observed under SEM, the surface of a lotus leaf contains a great number of microscale protruding nubs. Their function is to support the water droplet to minimize contact with the surface. On every single nub, there are millions of nanoscale arms working as the secondary roughness, which helps prevent the waterdrop from penetrating into the cavities between the nubs. When air is entrapped in these gaps, waterdrops can roll off the surface when it is slightly tilted. When rolling, the waterdrop is also able to clean away debris particles on the surface, which is known as the self-cleaning effect. In 2007, Wang and Jiang [9] suggested a standard for superhydrophobicity, which includes a contact angle greater than 150 degrees, and a tilt angle less than 10 degrees.

\subsubsection{Measurement of Contact Angle}

Contact angle measurement has been widely used to characterize surface wettability both physically and chemically. The most common technique being utilized is called sessile drop method, accomplished using a contact angle goniometer. A goniometer consists of a 
horizontal stage (Fig. 1.1.5 b), which can move in X-, Y- and Z-axes. Some advanced goniometers have four degrees of freedom including a rotational motion. The stage is located between a high-resolution video camera (Fig. 1.1.5 a) and a halogen light source (Fig. 1.1.5 c). Above the stage, a needle (Fig. 1.1.5 d) connected to a syringe (Fig. 1.1.5 e), which contains the liquid being used for the measurement, is used to dispense a droplet onto the specimen on the stage. The device shown below is a DataPhysics Optical Contact Angle goniometer (OCA) 35, which connects to a PC. All the images, database, and videos are generated by using the software SCA 20 for further analysis. The workpiece is usually tilted one to two degrees towards the camera to avoid blocking the contact line by the surface edge. After a clear image is taken, the contact angle can be measured manually or automatically. To measure it manually, software like ImageJ or MatLab are useful options. This thesis mainly focuses on computing the contact angles automatically by using SCA 20 software.

Contact angle measurement is required to be taken in an environment with a controlled humidity, temperature, and dust level. During the measurement process, the stage should be free of vibration or air flow. The surfaces being measured should be free of contaminants. Direct sunshine and bright lights should be avoided to extract clearer liquid droplet profile. Compared to other liquid solutions, water is more reliable in rough measuring conditions. Typically, a droplet volume from $1 \mu \mathrm{L}$ to $2000 \mu \mathrm{L}$ can be used for the measurement and there is no significant difference in the results [2]. A drop size in the range of $5 \mu \mathrm{L}$ to $10 \mu \mathrm{L}$ is recommended for measuring [11] surfaces with a high waterrepellency. After capturing the drop shape, the four most commonly used automatic fitting 
methods used to calculate contact angle are: circle fitting, ellipse fitting, tangential fitting, and Young-Laplace fitting.

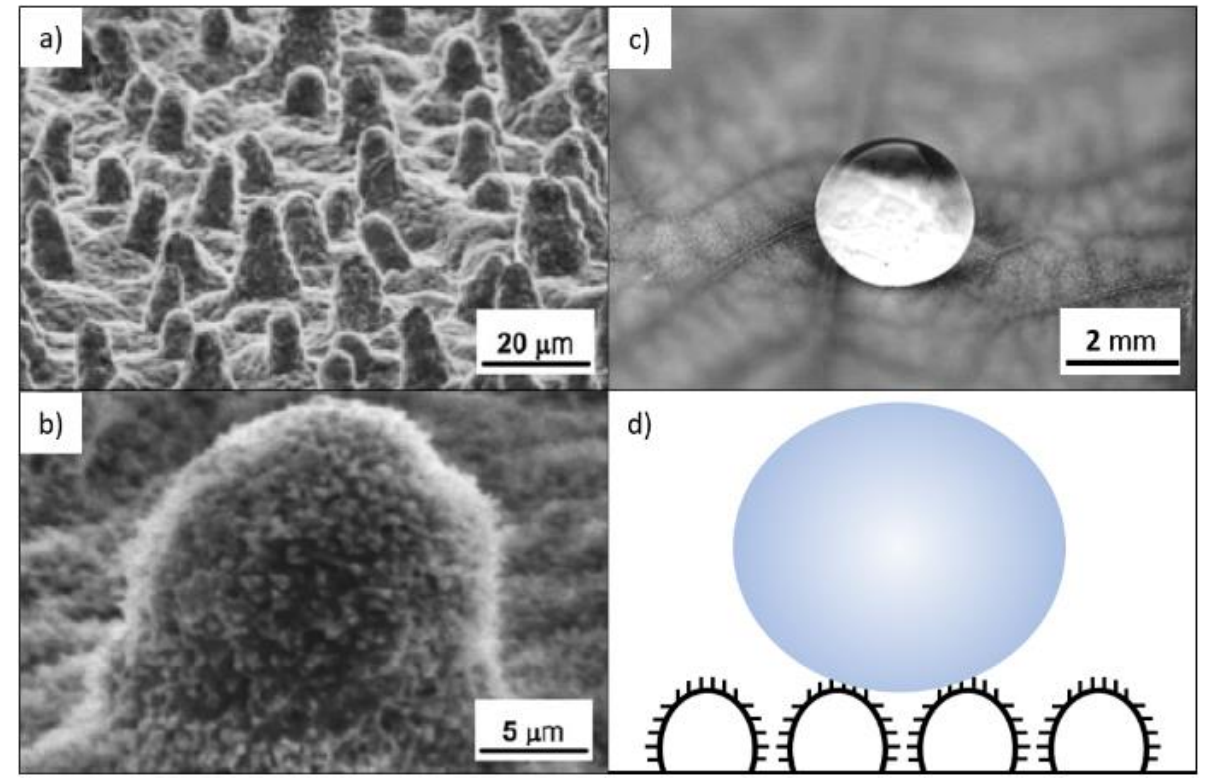

Fig. 1.1.4 a) SEM image of microscale protrusions and b) nanoscale protrusions on lotus leaves. c) A water droplet sitting on a lotus leaf. d) Schematic two-dimensional view of a water droplet sitting on the hierarchical structure [10].

Circle fitting assumes the droplet on the surface to be a part of a sphere, which neglects the effect of gravitational force. It is more suitable for a small drop volume. Ellipse fitting method is similar to circle fitting method but considers the droplet as a part of an ellipse. Static contact angle can be calculated with less error when the droplet has a relatively higher volume. However, as the ellipse equation is more based on the captured droplet profile, a large error may occur when the surface has a high hydrophobicity. 


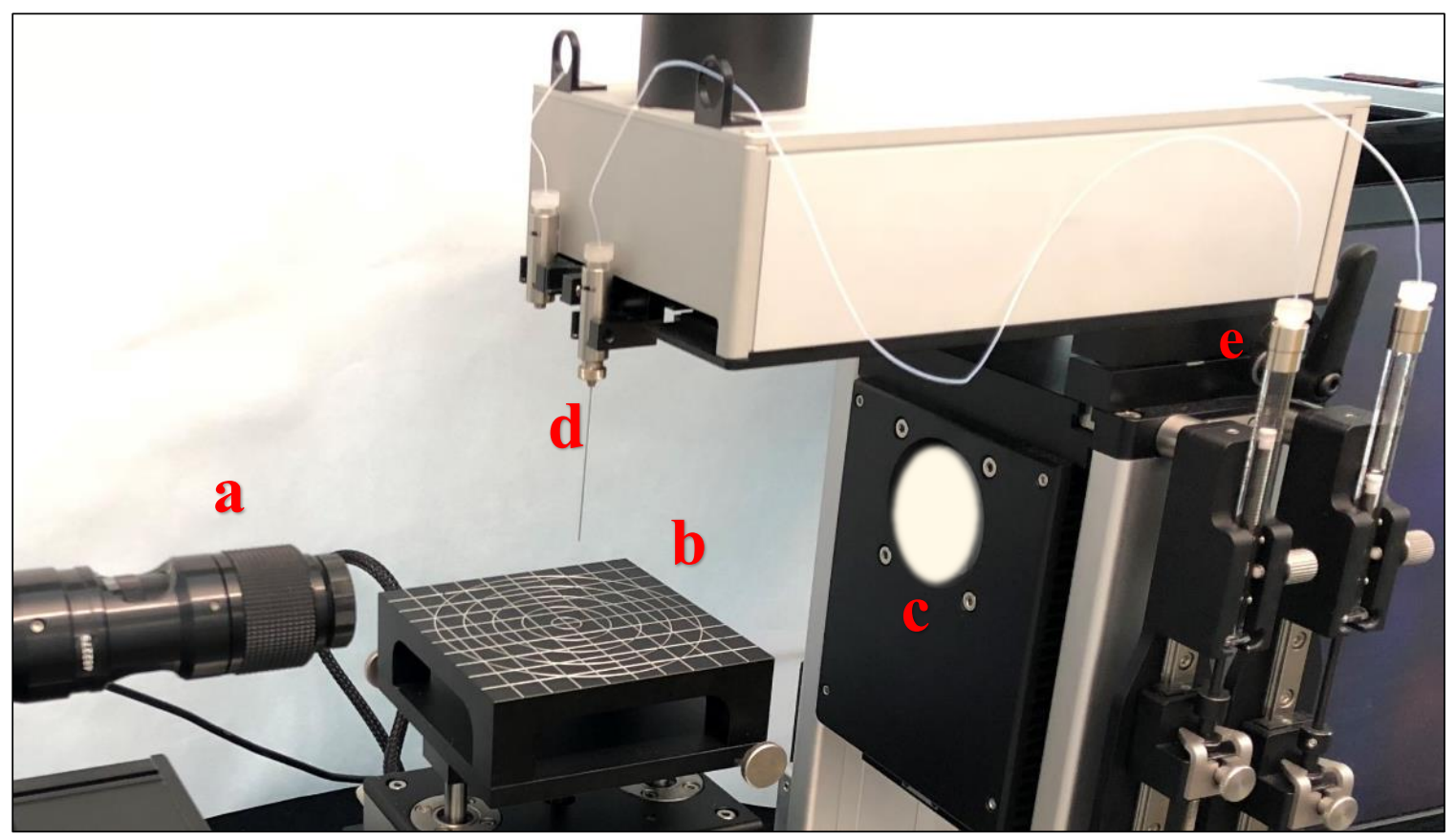

Fig. 1.1.5 OCA 35 High Speed Contact Angle Measurement Machine includes (a) a highresolution video camera, (b) a horizontal stage, (c) a halogen light source, (d) a removable needle, (e) and a microliter syringe.

Tangential fitting is based on the tangential line to the droplet and the contact point between the drop and the surface. This method is not influenced by the drop volume, or the general profile. However, surface defects and contaminations can greatly decrease the accuracy of the results. Young-Laplace fitting is the most common method in sessile drop analysis. The drop profile is generated based on the force equilibrium between the gravity and the surface tension of the sample. Contact angle can be calculated with a high repeatability if the droplet is highly axisymmetric. 


\subsection{Electrical Discharge Machining}

Electrical Discharge Machining (EDM) is a non-conventional machining process that is used to machine electrically conductive materials with precisely controlled sparks. Unlike conventional machining technologies, EDM can proceed without considering the hardness of the workpiece, but just the thermal properties. High strength materials can be machined by using EDM easily. Another difference is that there is no direct contact between the tool and the workpiece in EDM process, shown in Fig. 1.2.1, therefore the machining force is almost negligible which makes the machining process more stable and accurate.

EDM process works by using electricity as the power source. Before machining starts, the electrode and the workpiece are both connected to an electrical circuit with a gap in the middle (Fig. 1.2.1 (a)). The gap is filled by dielectric medium, which is usually a hydrocarbon oil in Sink EDM or deionized water in Wire EDM. When the machining starts, the electrode moves towards the workpiece until the gap reaches a certain value typically ranging from $10 \mu \mathrm{m}$ to $100 \mu \mathrm{m}$ [12]. A high voltage overcomes the dielectric breakdown strength and a plasma channel is generated (Fig. 1.2.1 (b)). Current flows through the channel during the discharge duration, which generates an extremely high temperature about $8000{ }^{\circ} \mathrm{C}$ to $12000{ }^{\circ} \mathrm{C}$ [13]. This temperature is much higher than the melting points of all the known materials, therefore the discharge can melt a small volume of some material from both the electrode and the workpiece, resulting in a small crater on each surface, and debris particles in the gap (Fig. 1.2.1 (c)). Because of the rapid rise in temperature, the electrode materials and the dielectric liquid are evaporated which causes 
a rapid bubble expansion, which helps remove the debris particles from the local area. The discharge appears in a very short period on the order of microseconds; therefore, the machine generates several hundred thousand of sparks in a second. After one cycle of machining, the electrode is retracted (Fig. 1.2.1 (d)), and the gap is cleaned by the flushing dielectric medium. It can also help to cool the surfaces and regenerate the dielectric breakdown strength, paving the way for the next cycle of discharges.

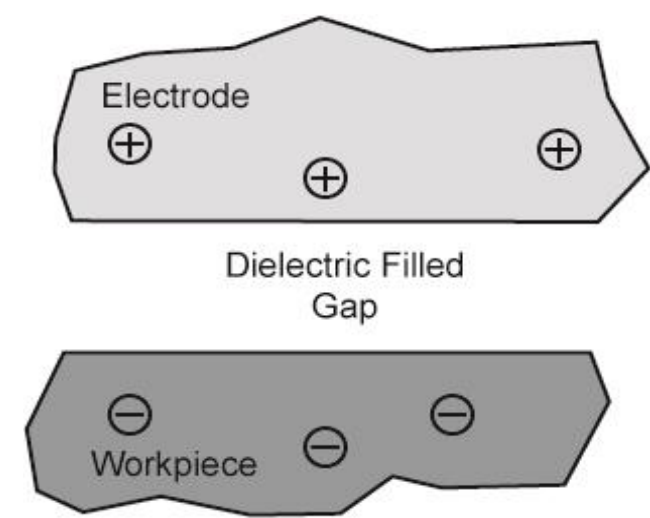

(a) Dielectric medium fills the gap between the electrode and the workpiece.

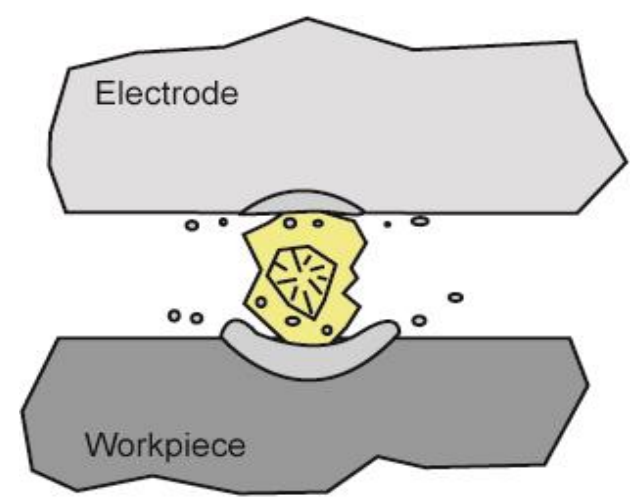

(c) Materials from both the electrode and the workpiece are melted, turning into debris particles.

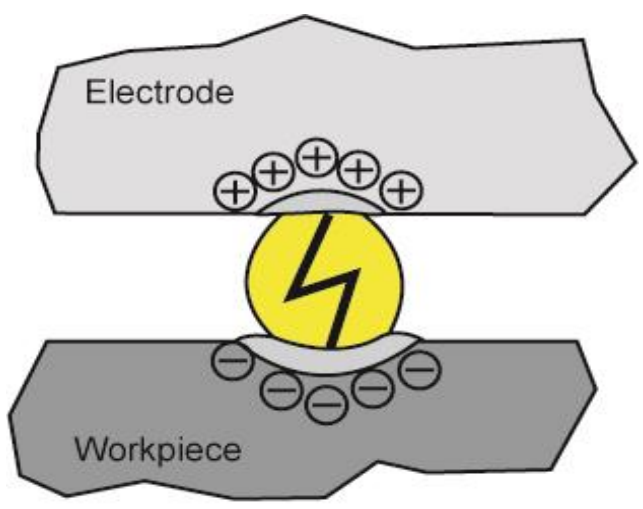

(b) A discharge is generated through a plasma chanel.

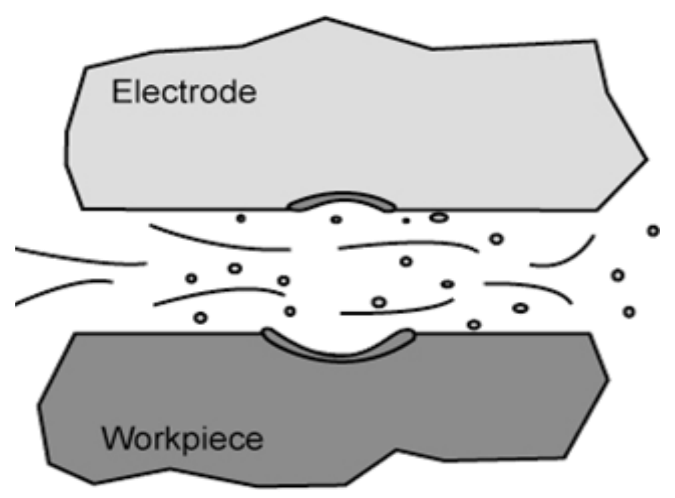

(d) Debris particles are flushed away, and the dielectric breakdown strength is regenerated.

Fig. 1.2.1 Four stages in a single EDM discharge. 
Due to the relatively low material removal rate, EDM is mainly used for manufacturing dies and tools with a complex geometry. It is also widely used in the aerospace field for delicate parts with special shapes that conventional machining cannot generate.

There are two types of EDM, Sink EDM (Fig. 1.2.2) and Wire EDM (Fig. 1.2.3). Sink EDM is a three-dimensional machining process, with a high accuracy and a surface finish up to $\mathrm{Rz}$ value of $0.4 \mu \mathrm{m}$ [12]. The workpiece can be machined to an opposite shape of the designed electrode, or "milled" by a rotating electrode with a cylindrical shape. Copper and graphite are commonly used as electrode materials, due to their high electrical conductivity, thermal conductivity and high melting point. Hydrocarbon oil is selected as the dielectric medium in Sink EDM because it can enhance surface finish and reduce tool wear. During machining process, the electrode and the workpiece are fully immersed in the oil, and the oil level should be at least $40 \mathrm{~mm}$ above the machining location to avoid a fire hazard. The crater size is determined by two parameters: current and pulse duration, which are controlled by the operator.

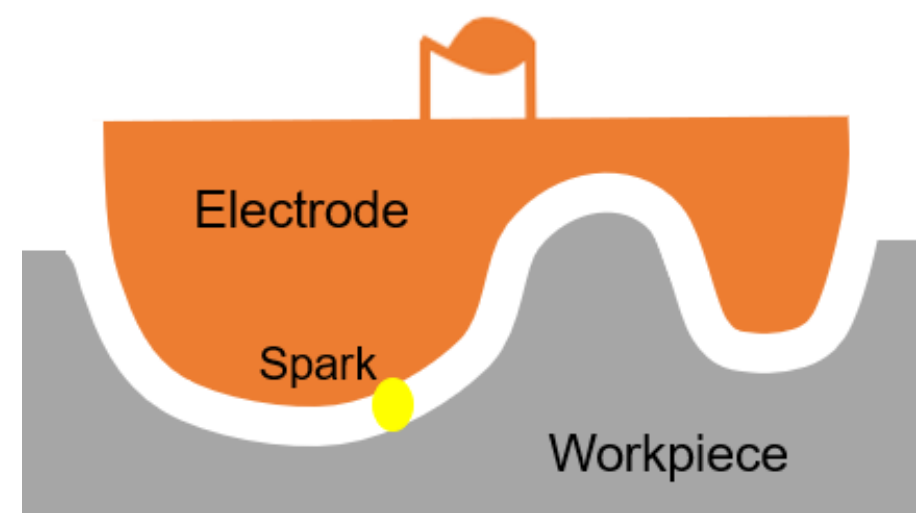

Fig. 1.2.2 Schematic view of Sink EDM process. 
There are some limitations of Sink EDM. The most obvious one is that to machine the workpiece, the tool electrode must to be of a complementary geometry and manufactured. Compared to conventional machining, the low material removal rate makes EDM only suitable for machining geometrically complex features. Sink EDM can furthermore only machine electrically conductive materials, which also applies to Wire EDM.

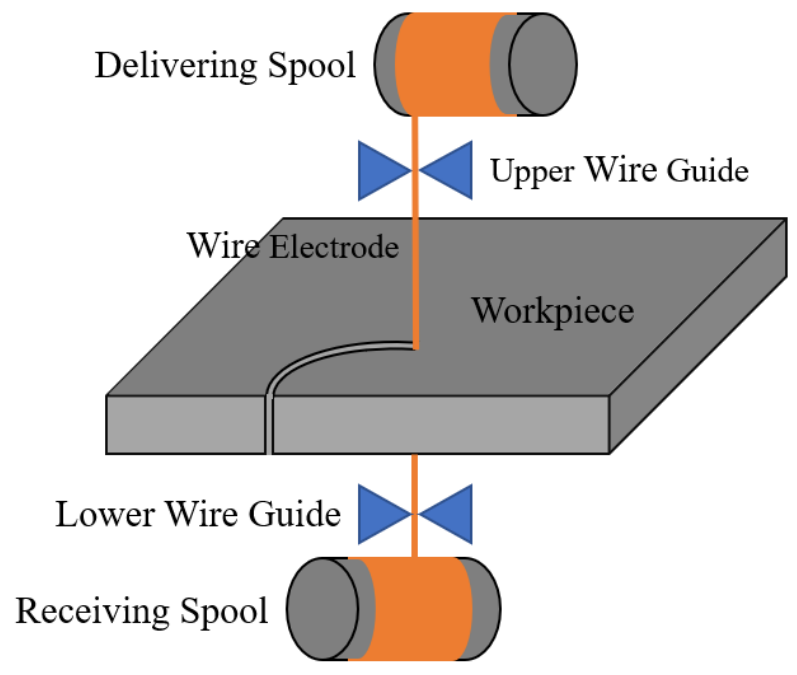

Fig. 1.2.3 Schematic view of Wire EDM process.

Wire EDM is generally a two-dimensional machining process, which uses a wire as an electrode. Typical wire thickness ranges from $20 \mu \mathrm{m}$ to $330 \mu \mathrm{m}$ [12]. Due to the small diameter, a delivering spool keeps feeding new wire to the machining zone to prevent wire breakage, and a receiving spool collects the used wire. These two spools also provide the wire with a tensile force which helps to minimize wire vibration and deflection. Copper is replaced due to its low tensile strength which can cause machining inaccuracy and breakage 
of the wire. As a result, brass, which is an alloy of copper and zinc, is chosen to be the wire material. The upper wire guide can be moved horizontally, so that the wire orientation can be altered to machine surfaces of different angles; however, due to the tension in the wire, only ruled surfaces can be machined.

\subsection{Scope of Thesis}

A novel technology of fabricating superhydrophobic surfaces by using Sink EDM was performed with the proof of concept. Influential machining parameters including current and discharge duration were varied to study the relationship between surface structures and wettability. A fine texturing was conducted on the top of rough surfaces, so that a comparison between monolithic structure and dual structure could be studied. Different durations of the fine texturing was inputted to study the effect of secondary machining time. Two grades of aluminum alloys were machined to test the influence in contact angles by changing workpiece materials. Same electrode surface was used to machine multiple workpieces which was to investigate if different contact angles would be obtained by using a used electrode. An experiment using deionized water instead of hydrocarbon oil was also conducted to study the effect of altering the dielectric medium. Inspired by other works, the change in surface wettability over time was also studied for a time span of 30 days. A PDMS casting process was finally accomplished to study the wettability of the opposite structure of EDM-generated surfaces, which might hold potential for a molding process of water-repellent polymeric surfaces. Furthermore, surface characteristics were analyzed by 
using surface profiles, Fast Fourier transforms, and surface chemical compositions to investigate the mechanism behind water repellency. High contact angles and low contact angle hysteresis were desired with the optimal machining parameters and conditions. 


\section{Chapter 2}

\section{Literature Review}

Superhydrophobicity has been studied for more than half a century. A unique surface structure and low surface energy are the most significant criteria needed for non-wettable surfaces. A great number of fabrication technologies have been reported, and high waterrepellent surfaces have been obtained. Variant structures are constructed by using different kinds of metals and polymers. This chapter provides an overview of different fabrication technologies of superhydrophobic surfaces.

Due to the large volume of the relevant literature, representative works done with machining technologies will be focused in this chapter. Other fabrication methods including electrochemical deposition, lithography, templating, plasma treatment, and chemical vapor deposition (CVD) based surface treatments can be found in review papers $[14,15,16]$.

\subsection{Laser Beam Machining}

Laser Beam Machining (LBM) is a non-conventional machining process which uses thermal energy to melt away materials from the workpiece. A general laser system contains a laser source, which provides the laser beam by exciting the electrons. The laser beam then goes through the attenuator to control the beam power. Then the beam diameter is expanded 
by utilizing a beam expander. When the expanded beam is converged by the focusing lens, a finer and smaller spot size can be generated onto the workpiece that is on the stage. A controller is usually used which can control both the laser beam supply and the movement of the stage. There is also a group of mirrors used to orient the beam directions within the entire path. A schematic view of the LBM process is shown in Fig. 2.1.1.

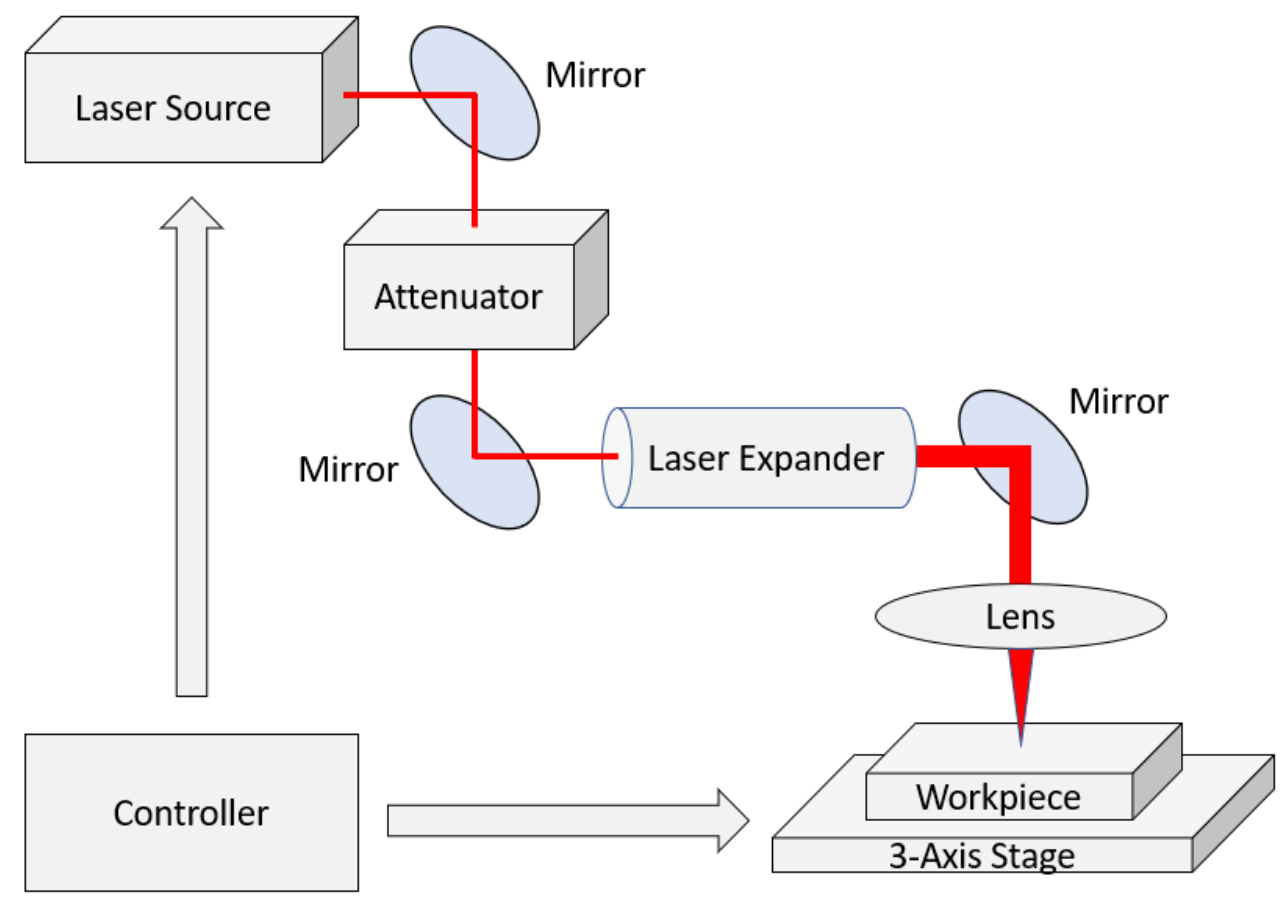

Fig. 2.1.1 Schematic view of LBM process.

LBM has been widely used for a variety of scientific researches due to its high precision and accuracy, and wide range of workpiece material selections. Its ability to machine micro and even nano level features attracts a great amount of attention in surface texturing technologies. Fabricating superhydrophobic surfaces by using LBM has become very 
popular in the recent years. Both metallic and non-metallic materials have been attempted, and high water repellent surfaces can be successfully obtained.

\subsubsection{LBM of Metallic Superhydrophobic Surfaces}

As mentioned in the first Chapter, a low surface energy is one of the most significant criteria needed for creating superhydrophobic surfaces. Metallic surfaces, also known as high energy surfaces [17], are not suitable for this unique application. However, due to their higher strength, longer durability, and wider range of applications, fabrication of a metallic superhydrophobic surface attracts a great amount of interest all over the world. LBM is the most popular technique currently being used.

Kietzig et al. [18] reported a method for creating metallic superhydrophobic surfaces by using Femtosecond Laser Irradiation. Six types of materials, including stainless steel AISI 304L and 630, low alloy steel AISI 4140, high speed steel AISI M2, mold steel AISI P20 with chromium coating, and Titanium alloy Ti6Al4V, were machined with varied fluences. They observed that a fine ripple structure was formed on the top of many big bumps. With an increased fluence, the scale of the bumps increased. They believed this double-scaled structure was working as a hierarchical structure which could help improve hydrophobicity. Directly after the machining process, all surfaces were completely wetted showing superhydrophilicity. Contact angles on all the samples increased over time, which showed a similar change as many other reports $[19,20,21,22,23,24]$. After 5 to 10 days, the contact angle remained the same for all surfaces, which indicated a high hydrophobicity 
around $130^{\circ}$ to $150^{\circ}$ with a fluence value of $0.78 \mathrm{~J} / \mathrm{cm}^{2}$ (Fig. 2.1.2). Surface chemistry was analyzed by using X-ray photoelectron spectroscopy (XPS), which pointed to a rise in carbon level to be the factor responsible for this phenomenon. Superhydrophilicity immediately after machining was due to the formation of metallic oxides which are usually very hydrophilic.

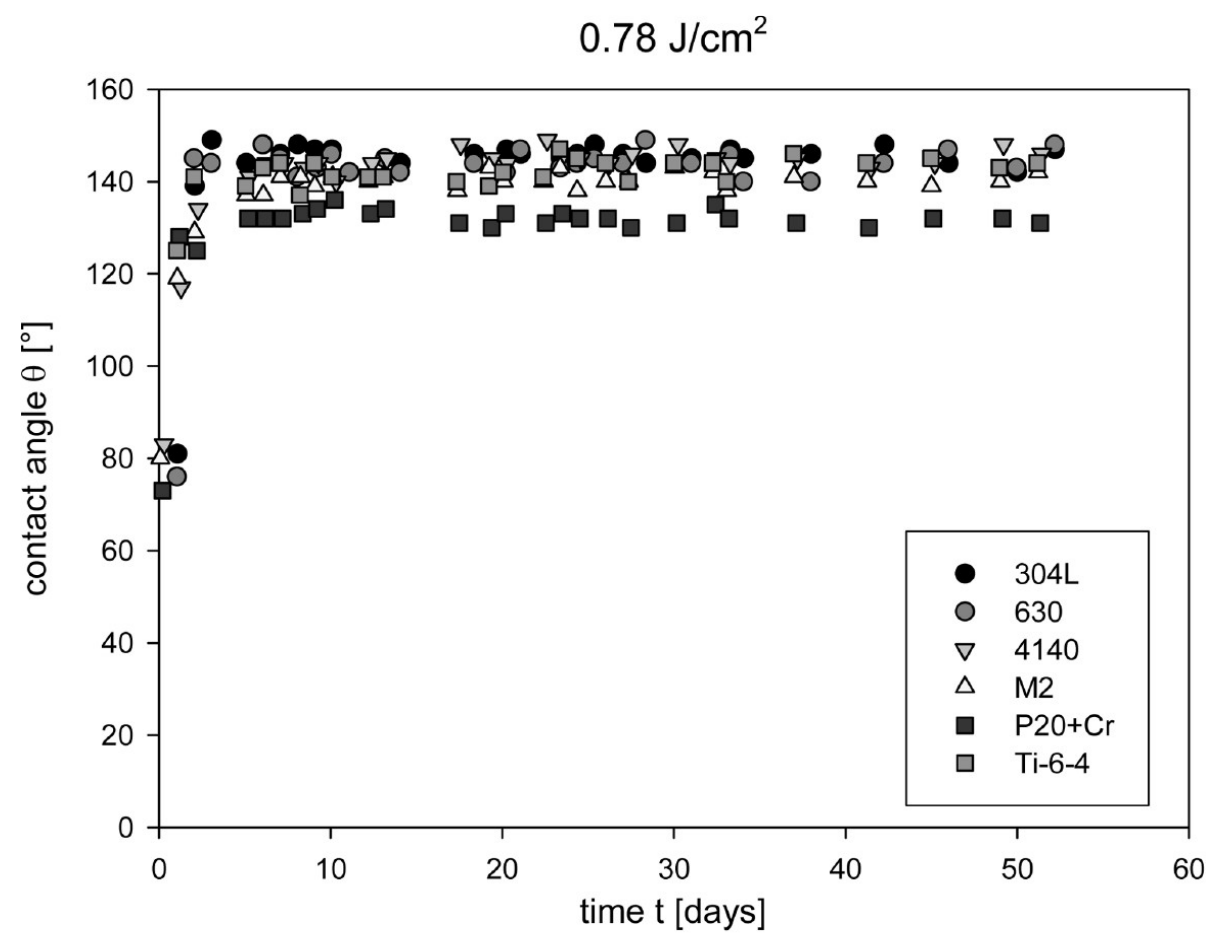

Fig. 2.1.2 Contact angle change over time for six materials with a laser fluence of $0.78 \mathrm{~J} / \mathrm{cm}^{2}$ [18].

Similar works were accomplished by Wu et al. [25] and Li et al. [22], different laser fluences were attempted on pure titanium and stainless steel respectively. Instead of monitoring the wettability change over time, workpiece samples were coated after the machining process. Wu's group reported a high fluence laser machining, which fabricated 
micro-scaled periodic cone-shaped spikes covered by laser-induced periodic surface structures (LIPSS) in nano scale (Fig. 2.1.3 left). To reduce the surface free energy, a 70minute silanization process was followed by using Trichloro silane $\left(\mathrm{HSiCl}_{3}\right)$ in a vacuum condition. Compared to a flat surface with a contact angle of $113.0^{\circ} \pm 0.9^{\circ}$, a value as high as $166.3^{\circ} \pm 1.1^{\circ}$ was obtained on the surface machined with laser fluence of $2.4 \mathrm{~J} / \mathrm{cm}^{2}$ (Fig. 2.1.3 right). Li's group also investigated the difference between surfaces with and without coating after the laser texturing. With a fluence of $1.5 \mathrm{~J} / \mathrm{cm}^{2}$ on titanium surfaces, superhydrophobicity (contact angle $163.7^{\circ} \pm 1.1^{\circ}$ with $\mathrm{CAH} 3.3^{\circ}$ ) and superhydrophilicity (contact angle $0^{\circ}$ with no $\mathrm{CAH}$ ) were both observed on the surfaces with or without silanization treatment respectively. The effect was also studied over a time span of 30 days, and similar results were obtained which meant the wettability of the surfaces was stable.

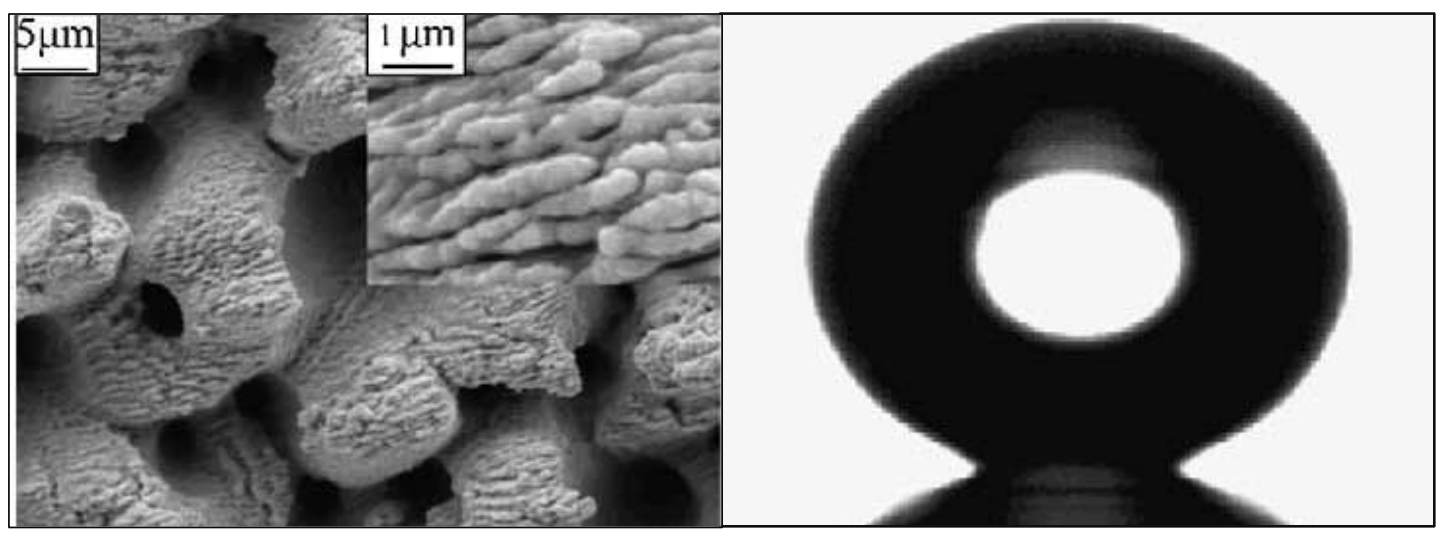

Fig. 2.1.3 (left) SEM of laser machined AISI 316L Stainless Steel with fluence of $2.4 \mathrm{~J} / \mathrm{cm}^{2}$, (right) and a water drop profile on its surface with a contact angle of $166.3^{\circ} \pm 1.1^{\circ}$ [25]. 
Different to the two papers mentioned above, Jagdheesh et al. [26] coated a layer of perfluorinated octyltrichlorosilane (FOTS) to reduce the surface free energy of AISI 304L stainless steel and Ti6Al4V alloy workpieces. In addition, the number of pulses per laser irradiation spot was investigated as the variable instead of the fluence. With a machining of 48 pulses with a spot size of $18 \mu \mathrm{m}$, a contact angle of $152^{\circ} \pm 3^{\circ}$ was observed on titanium alloy surfaces, and $140^{\circ} \pm 3^{\circ}$ on stainless steel surfaces. A low CAH of 2 to $4^{\circ}$ was also measured on the former surface.

To generate a grid pattern by using LBM was another approach in this research field. Ta et al. [19] demonstrated the wettability of stainless steel surfaces in a function of fluences, by laser scanning the surfaces in both $\mathrm{x}$ and $\mathrm{y}$ directions. Hydrophilicity was observed in the first six days, and after 12 days, surfaces went into the superhydrophobic regime. Chun and Ngo et al. [20, 24] investigated the influence of the step size between laser scanning lines. Initial hydrophobicity was transformed by a low temperature annealing post treatment. The post process was conducted at $100^{\circ} \mathrm{C}$ in an oven for about 4 hours. An increase in elemental carbon was still believed to be the key factor that raised the contact angle of stainless steel samples. Nevertheless, the form the metallic oxides was concluded to be the reason for lasered machined copper surfaces transitioning from hydrophilicity to superhydrophobicity. The low temperature annealing process accelerated the transformation from cupric oxide ( $\mathrm{CuO}$ hydrophilic material) to cuprous oxide $\left(\mathrm{Cu}_{2} \mathrm{O}\right.$ hydrophobic material) shown in Fig. 2.1.4, which shortened the wettability transition from more than 30 days to just several hours. 


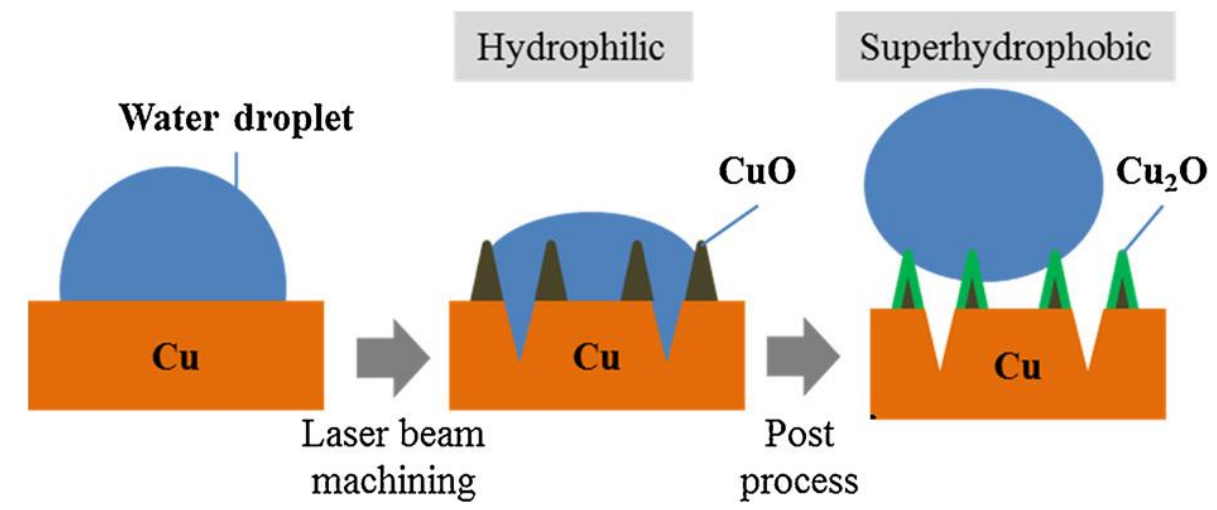

Fig. 2.1.4 Schematic view of the wettability transition by LBM and the post process [20].

\subsubsection{LBM of Non-Metallic Superhydrophobic Surfaces}

Compared to metallic materials, non-metallic surfaces usually have lower surface free energy which means they are intrinsically more hydrophobic. Farshchian et al. [23] used polydimethylsiloxane (PDMS), which was one of the most hydrophobic materials, as the workpiece. A grid pattern with different step sizes were constructed to investigate the influence in contact angles. Immediately after the laser machining process, all the surfaces still indicated hydrophilicity due to the generation of some high energy oxides, and decomposition of PDMS. However, hydrophobicity could be recovered gradually without any post treatment. After five hours, a contact angle of $154^{\circ} \pm 2^{\circ}$ with a sliding angle of $4^{\circ} \pm$ $2^{\circ}$ could be obtained.

Chun's group, mentioned in last section, also conducted a similar experiment by utilizing sapphire as the workpiece material [21]. Same as their previous works, a lasered grid pattern followed by a low temperature annealing post process was conducted. A 
contact angle as high as $176^{\circ}$ with a tilt angle of $4^{\circ}$ was obtained which indicated an extremely high water repellency. The roughened sapphire surfaces, after the machining process, maintained their transparency which made the technology better applicable in industrial designs.

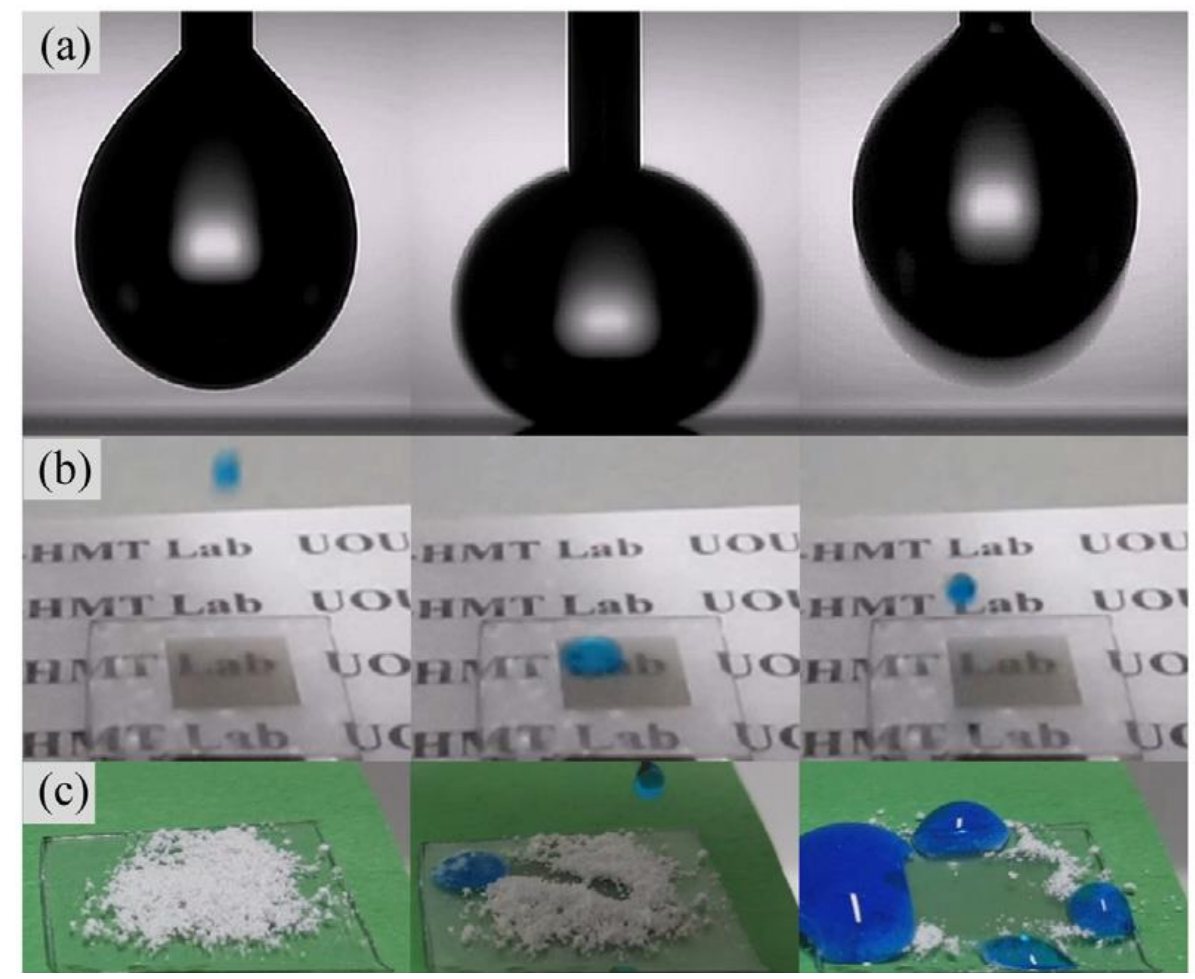

Fig. 2.1.5 Performance of the laser textured sapphire surface: (a) high water repellency (b) high transparency (c) self-cleaning effect [21].

\subsection{Electrical Discharge Machining}

EDM, which is the focus in this thesis, has also been used for fabricating superhydrophobic surfaces. The low machining speed is one of the limitations of EDM process; however, 
surface texturing does not require much material removal and so this limitation is of no consequence. Surfaces made up of peaks and valleys help support the water drop, so that the contact between them can be significantly reduced.

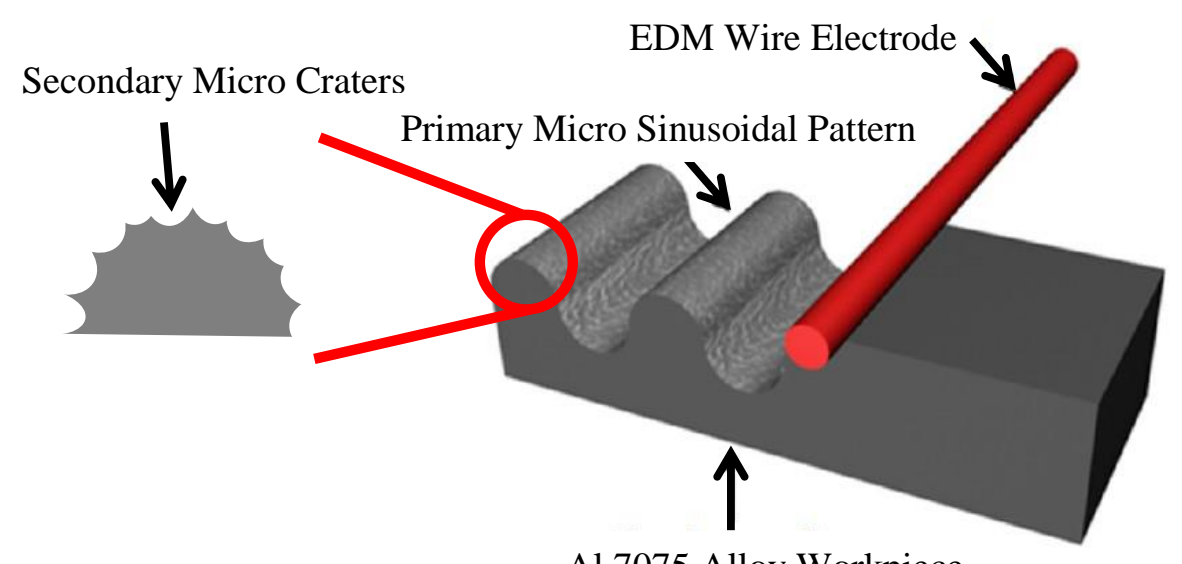

Al 7075 Alloy Workpiece

Fig. 2.2.1 Schematic view of Wire EDM simultaneous dual-structure fabrication process. (modified from [27]).

\subsubsection{Wire EDM of Superhydrophobic Surfaces}

Bae et al. [27] reported a one-step Wire EDM technique that could fabricate directional superhydrophobic surfaces on aluminum 7075 alloy substrates. A designed sinusoidal pattern was textured as the primary roughness, and the craters generated by the wire electrode worked to generate the secondary roughness (Fig. 2.2.1). The two roughness scales formed the hierarchical structure which played a significant role in revealing the superhydrophobic effect. A contact angle of $156^{\circ} \pm 5^{\circ}$ with a CAH less than $3^{\circ}$ was obtained 
by setting the sinusoidal wavelength to be $500 \mu \mathrm{m}$. Mechanical robustness of the surface was also tested by scratching it with a P3000 sandpaper, and the contact angle barely changed. A polymer surface, made of perfluoropolyether (PFPE) replicated by using lithography, was tested using the same method, and the contact angle dropped from $165^{\circ}$ to $15^{\circ}$. This experiment clearly indicated the durability of metallic surfaces from mechanical damage.

Furthermore, they used this technique for a real life roller mold application [28]. Instead of flat surfaces, the same machining process was constructed on a cylindrical rod which could be used as roller molds. A superhydrophobic polymer film, with the opposite patterned surface, could be easily manufactured (Fig. 2.2.2 Left).

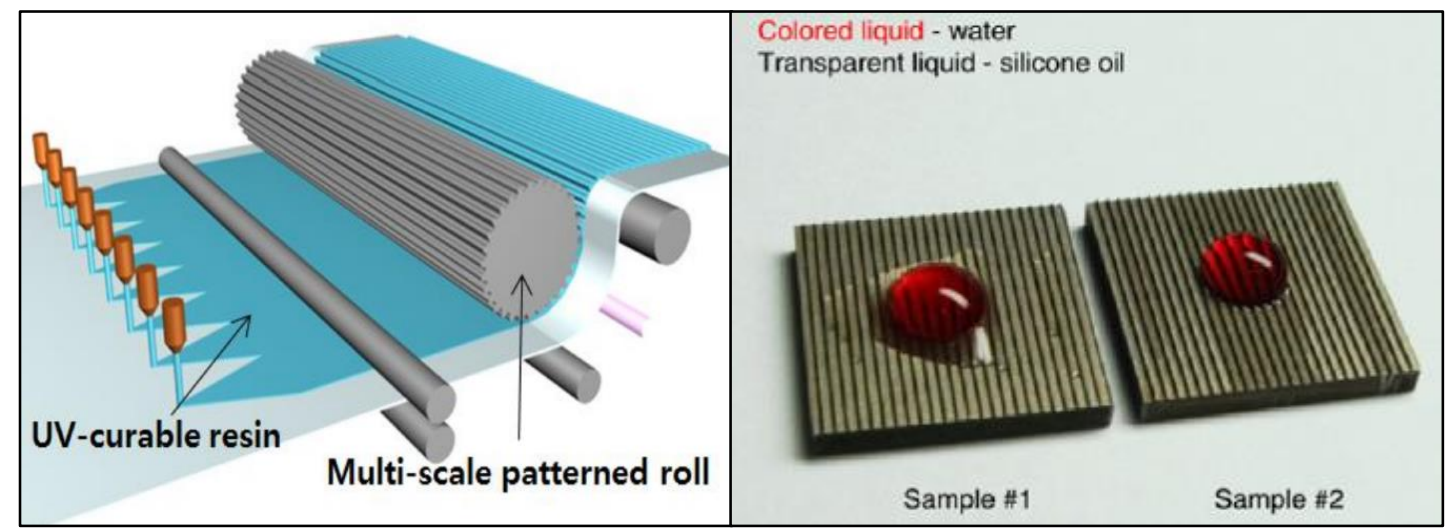

Fig. 2.2.2 (left) Schematic illustration of superhydrophobic polymer sheets manufacturing process by using Wire EDM textured roller [28], (right) and the effect water-oil separation on two stainless steel samples with different sizes of grooves textured by using Wire EDM [29]. 
The same group of researchers also demonstrated a method for fabricating superhydrophobic stainless steel surfaces by using Wire EDM [29]. A groove pattern was simply machined with different width and depth combinations. Grooves on workpiece sample \#1 were $100 \mu \mathrm{m}$ deep and $600 \mu \mathrm{m}$ wide, and the contact angles were $144.3^{\circ}$ and $53.3^{\circ}$ for water and silicone oil respectively. The sample \#2 with a groove depth of $200 \mu \mathrm{m}$ and width of $500 \mu \mathrm{m}$ had a water contact angle around $162^{\circ}$ and a silicone oil contact angle of $0^{\circ}$. This unique superhydrophobic and superoleophilic behaviour could be utilized for water-oil separation, which indicated significant industrial potential.

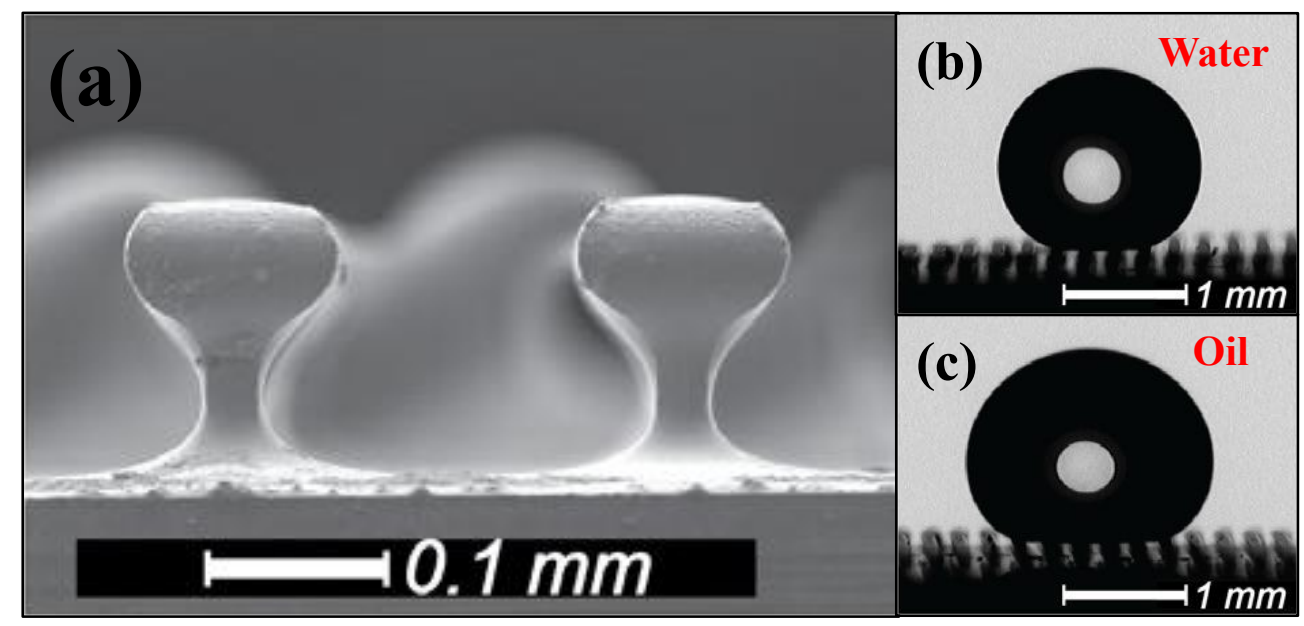

Fig. 2.2.3 (a) An example of the re-entrant microstructure machined by using micro Wire EDM, with (b) a water drop sitting on it and (c) a RL-68H oil sitting on it [30]

Different from Bae's work, Weisensee's group [30] reported a series of re-entrant microstructures textured on steel surfaces that indicated both hydrophobicity and oleophobicity (Fig. 2.2.3). Micro wires were used to machine in both $\mathrm{x}$ and $\mathrm{y}$ directions, so 
that the surfaces consisted of many micro-mushroom geometries. A Teflon layer was coated as a post treatment, which lowered the energy of the surfaces. The highest contact angles were $162^{\circ}$ with a $\mathrm{CAH}$ of $19^{\circ}$, and $152^{\circ}$ with a $\mathrm{CAH}$ of $70^{\circ}$ for water and oil respectively.

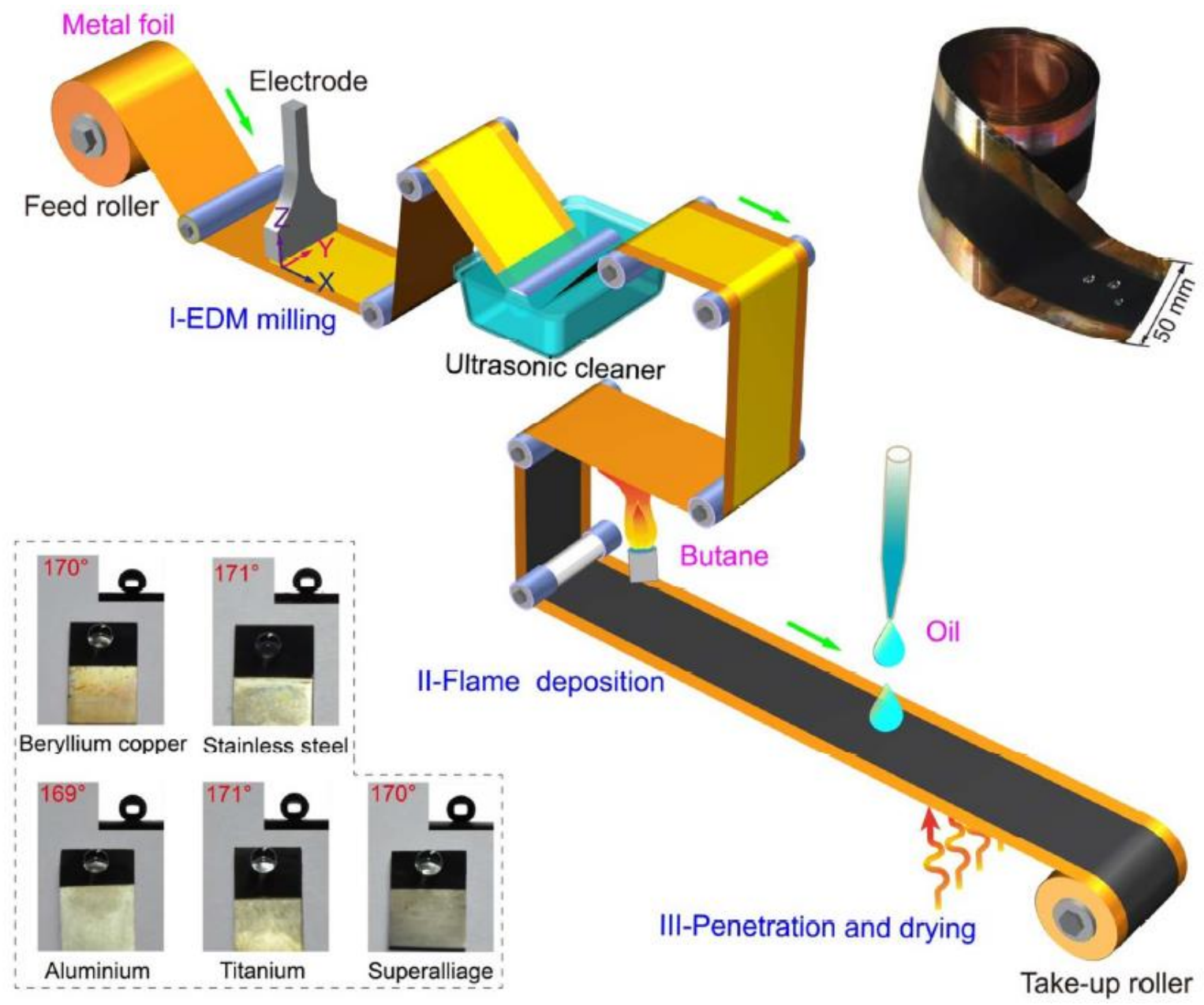

Fig. 2.2.4 A schematic illustration of the 3-step Sink EDM fabrication process of superhydrophobic surfaces [31]. 


\subsubsection{Sink EDM of Superhydrophobic Surfaces}

Dong et al. [31] demonstrated a three-step Sink EDM process which could fabricate superhydrophobic surfaces by using a variety of materials. The primary roughness texturing was constructed by EDM milling, followed with an ultrasonic cleaning process. Then the carbon nanoparticles from the flame of butane were coated onto the workpiece surfaces as the secondary roughness. Finally, kerosene oil was dipped onto the surfaces and then dried to help enhance the strength of the bond between carbon nanoparticles and the sample surface. Different materials were attempted, and high value of contact angles could be obtained for all of them. Robustness of the surfaces was tested by both water erosion and sandpaper scratching. Only high strength sandpaper scratching could reduce the contact angle about $20^{\circ}$ to $30^{\circ}$, which indicated a high resistivity to mechanical damage.

\subsection{Sandblasting of Superhydrophobic Surfaces}

As another one of few technologies that can generate surfaces with a positive skewness, sandblasting is also suitable for superhydrophobic texturing. Zhang et al. [32] demonstrated a three-step process to fabricate a hierarchical superhydrophobic surface. Sandblasting followed by a surface oxidation process was used for copper surfaces, and a fluoroalkylsilane (FAS) layer was coated to lower the surface free energy. The primary roughness gained from sandblasting and the $\mathrm{CuO}$ nanosheets generated from the oxidation process formed the hierarchical structure, which showed a high water-repellency with a 
contact angle of $161^{\circ}$ and tilt angle of $1^{\circ}$. Without the surface oxidation, contact angles of more than $140^{\circ}$ could still be achieved by sandblasting and the FAS modification only.

Another interesting experiment published by Shen et al. [33] introduced three different secondary texturing structures on the top of the primary texturing generated by sandblasting. Ti6Al4V alloy was selected to be the workpiece material in this research. After the sandblasting process, nanostructures including nanowire, nanotube and nanomesh were fabricated by three different techniques, anodic oxidation, hydrothermal method, and two-step chemical etching respectively (Fig.2.3.1). Finally, same as the previous literature, surfaces were modified with FAS to reduce the surface energy. They concluded that the nanowire structure constructed the highest water-repellency with a contact angle of $161^{\circ}$ and a sliding angle of $3^{\circ}$.

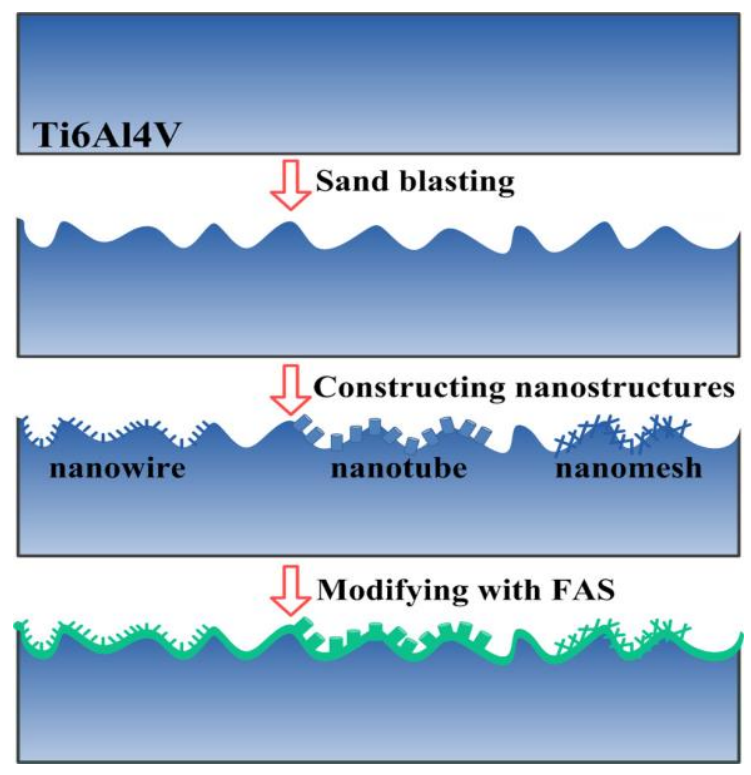

Fig. 2.3.1 Schematic illustration of three-step sandblasting process to fabricate superhydrophobic surfaces [33]. 
Victor et al. [34] introduced a molding method to fabricate polymeric superhydrophobic surfaces. The mold was made of electroformed nanocrystalline nickel coupons, and sandblasting technique was used to generate the primary roughness onto the surfaces, followed by a 30 -minute chemical etching process in a $5 \%$ nitric acid solution to generate finer scaled features. The textured nickel surfaces were simply compressed onto the heated polymers, which could replicate the complementary shape onto the polymer surfaces as shown in Fig. 2.3.2. A contact angle of $166^{\circ} \pm 3^{\circ}$ and a tilt angle smaller than $5^{\circ}$ could be achieved.

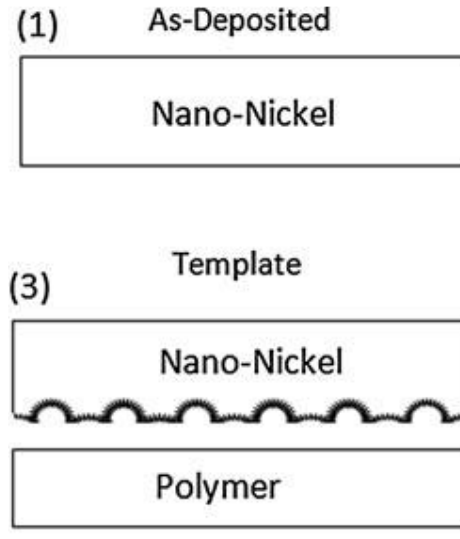

(5) Reusable Template

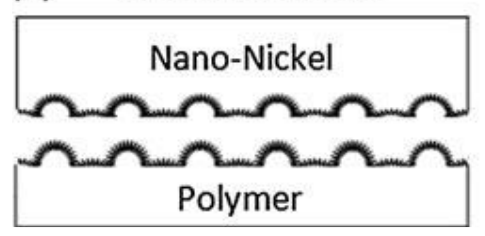

(2) Roughened Surface
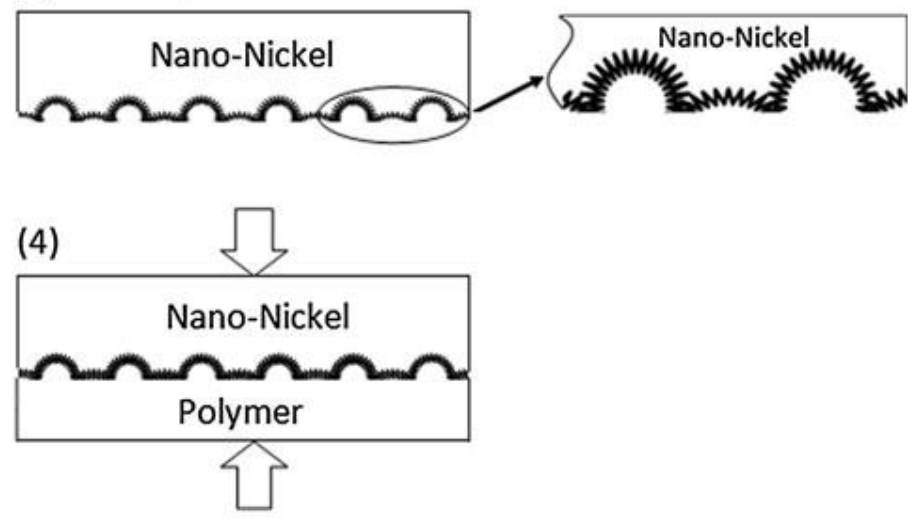

(6) Structured Surface

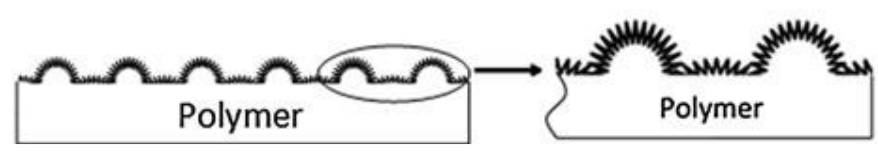

Fig. 2.3.2 Manufacturing process of polymeric superhydrophobic surfaces by using Nickel mold fabricated from sandblasting and chemical etching [34]. 


\subsection{Grinding and Cutting Processes}

Hydrophobic/superhydrophobic surfaces have been fabricated also by mechanical roughening technologies including grinding $[35,36]$, and micro-milling $[37,38,36]$. Fu and $\mathrm{He}[35]$ reported a method combining grinding and chemical etching to generate a hierarchical structure on Aluminum 8011 alloy substrates. A dodecyltrichlorosilane (DTS) modification process was constructed to enhance the carbon and silicon level, so that the surface free energy could be remarkably reduced. The contact angle was increased from $82.1^{\circ}$ on a flat surface to $159.7^{\circ}$ on a modified surface.

Shi et al. [37] demonstrated micro-milling method to cut micro grooves on PMMA and Ti6Al4V alloy surfaces. With a groove width of $50 \mu \mathrm{m}$, a contact angle of $138^{\circ}$ was observed on PMMA surfaces, and $135^{\circ}$ on titanium alloy surfaces. Yu et al. [36] did a micro-grinding process on aluminum alloy surfaces, which increased the contact angle to $162^{\circ}$. Guo et al. [38] reported an elliptical vibration cutting process which could achieve a dual-scale structure within one step. The cutting tool vibrates in an elliptical trajectory, so that cusps that work as the secondary roughness, can be generated along the cutting direction. Fig. 2.4.1 shows the surface profile of the machined Aluminum 6061 workpieces. The largest contact angle obtained was about $130^{\circ}$.

It is worth to mention that all the contact angles mentioned for micro groovepatterned surfaces are observed in a direction perpendicular to the grooves. The convex shape prevents the water drop from spreading; however, the water drop can still spread 
along the channel which is shown in Fig. 2.4.2. The contact angles observed along the groove direct are usually $20^{\circ}$ to $30^{\circ}$ lower than the ones perpendicular to it.

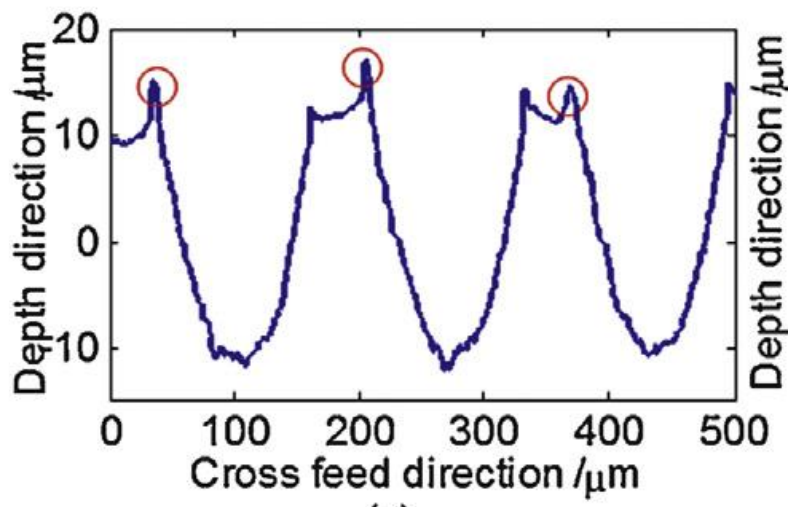

(a)

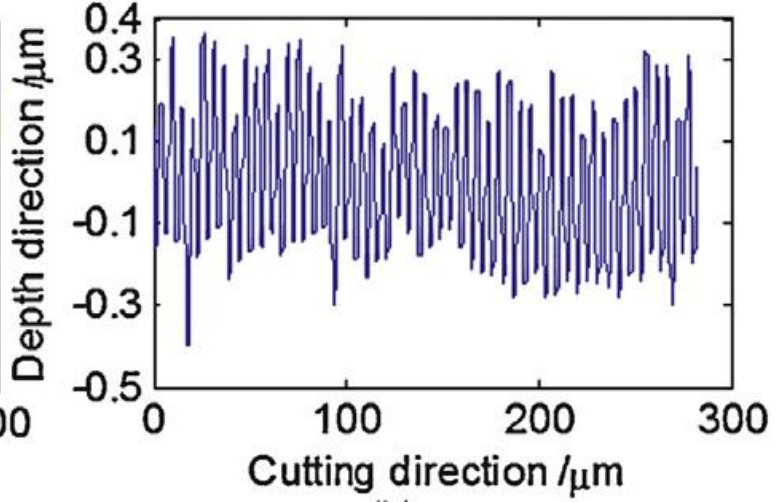

(b)

Fig. 2.4.1 Surface profile of the workpiece cut by ultrasonic elliptical vibration cutting (a) in the feed direction and (b) the cutting direction [38].

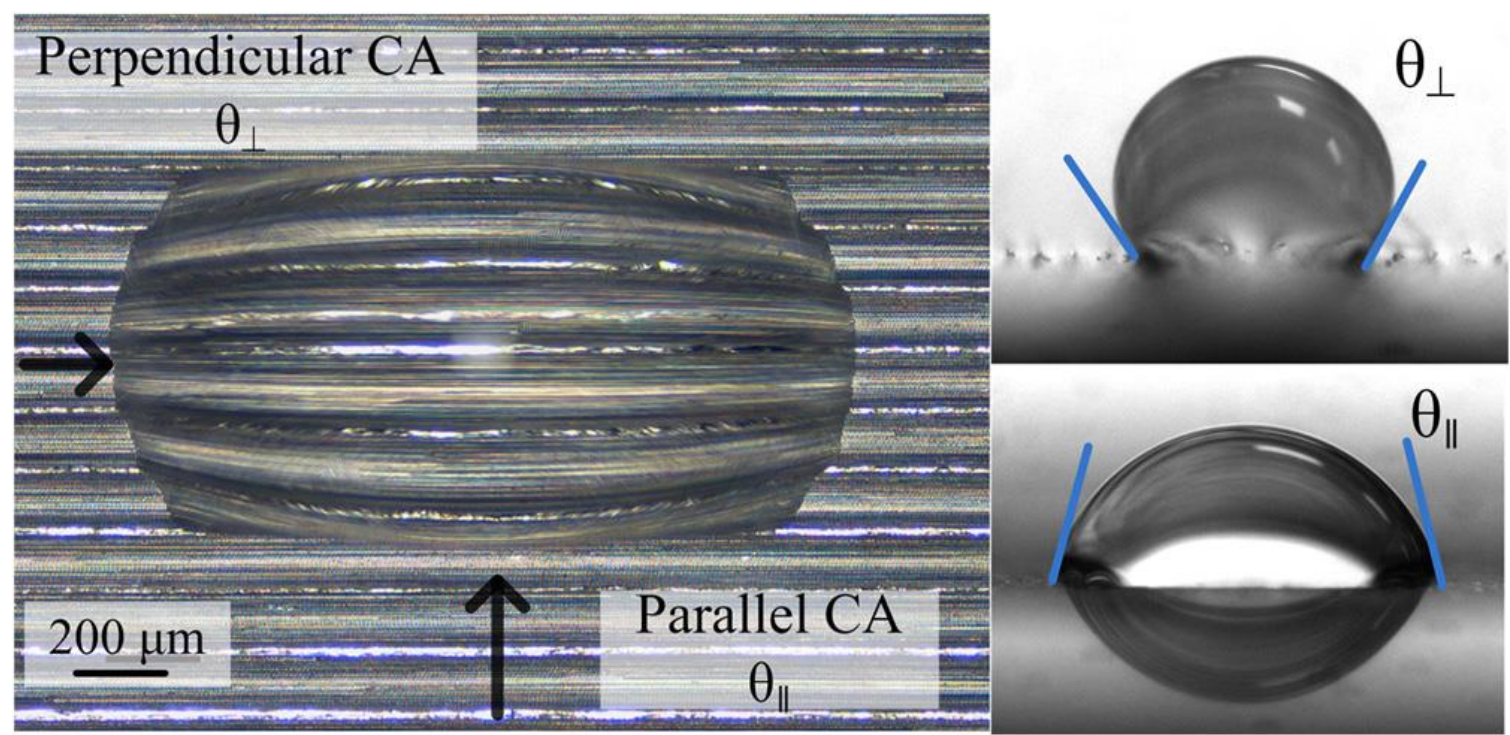

Fig. 2.4.2 An example of observing contact angle from different directions [38]. 


\subsection{Summary}

Superhydrophobic surfaces have a significant potential for both commercial applications and research studies. Many fabrication technologies were introduced in this chapter. LBM fabricates such surfaces by controlling the fluences on different materials or generating grid patterns with certain step sizes. Typically, hydrophilic or superhydrophilic surfaces are obtained immediately after machining. At least half of a month is needed for the surfaces to become superhydrophobic. A post-treatment like low temperature annealing or coating can enhance the water repellency in a shorter time. EDM processes utilize the designed patterns, discharged craters and particle depositions to form the hierarchical structures. Sandblasted surfaces usually need a post treatment like oxidation process, nano-structure deposition or chemical etching to make them superhydrophobic. Micro-milling method generates different groove patterns to prevent the water droplet from spreading in the perpendicular direction. Grinding process and vibration assisted cutting can help build a secondary roughness to further enhance the water repellency.

To fabricate a water-repellent surface for an area of $10 \mathrm{~mm} * 10 \mathrm{~mm}$, LBM needs at least five hours but only five to ten minutes by using EDM which indicates a significant difference in terms of time consumption. In this thesis, a Sink EDM method is introduced to generate metallic superhydrophobic surfaces without any post treatments. Different spark energies, dielectric mediums, and workpiece materials are investigated for the influence on hydrophobicity. Some surface characterization techniques are utilized to study the science behind the phenomenon. 


\section{Chapter 3}

\section{Experimental}

This research comprises fabrication of textured surfaces, measurement of contact angle and contact angle hysteresis, and surface characterization including topographic and chemical analysis. Most of the fabrication processes were conducted by using Sink EDM; therefore, the setup of EDM will be described first in this chapter. Grinding and polishing were conducted to obtain flat surfaces for intrinsic contact angle measurement. Cleaning methods were then introduced with the explanation of their influences. PDMS casting process was studied due to its potential of industrial purposes. OCA 35 goniometer contact angle measurement will be described followed by contact angle hysteresis measurement. Surface characterization techniques including confocal microscopy, scanning electron microscopy and high speed camera will be introduced at the end of the chapter.

\subsection{Fabrication}

\subsubsection{Sink Electrical Discharge Machining}

An AGIETRON Impact 2 Sink EDM machine, shown in Fig. 3.1.1, was the machine tool used in this thesis research. The drive system, controlled by the high performance brushless AC servomotors, contains four degrees of freedom; three linear motions and one rotational motion with the finest step size of $0.1 \mu \mathrm{m}$ and $0.1 \mu^{\circ}$ respectively. The traversing range of 
the working tank is $350 \mathrm{~mm}, 250 \mathrm{~mm}$, and $350 \mathrm{~mm}$ for X-, Y-, and Z-axis respectively, with the highest linear speed of $1500 \mathrm{~mm} / \mathrm{min}$. An Intelligent Power Generator (IPG) can provide a wide range of current from $1.2 \mathrm{~A}$ to $72 \mathrm{~A}$, with the smallest pulse duration being $0.4 \mu \mathrm{s}$.

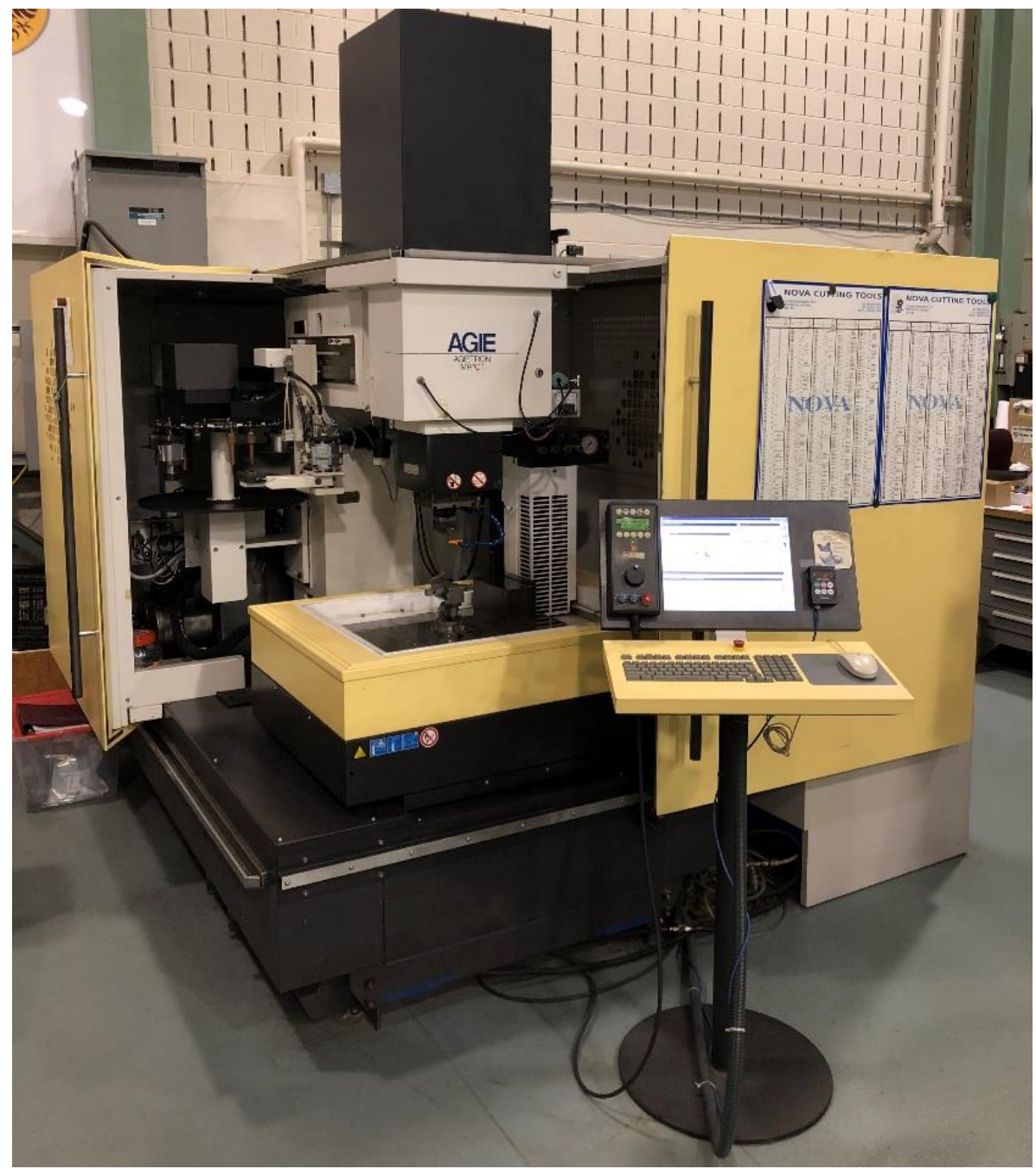

Fig. 3.1.1 The AGIETRON Impact 2 Sink EDM. 
System 3R Macro chucks are connected to the quill and held by compressed air. Different electrodes with customized shapes can be easily clamped, and the maximum weight that the chuck bears can be as high as $100 \mathrm{~kg}$. About $400 \mathrm{~L}$ of hydrocarbon oil was used as the dielectric medium, which helps to enhance the material removal rate, reduce the electrode wear, and generate the dielectric breakdown strength. A 1.5 bar high pressure flushing is supplied through a thread pipe hose, which precisely aims at the working zone to improve the machining performance. An external chiller is used to cool down the dielectric medium so that the machining temperature can be well maintained.

During the machining process, the Adaptive Control Constraints (ACC) and the Adaptive Control Optimizations (ACO) help to monitor and optimize the erosion gap conditions so that a stable machining operation can be achieved.

Electrode material used in this research was primarily pure copper, which is the most common tool material used for Sink EDM. Aluminum Alloy 7075 was selected to be the workpiece material because of its low surface free energy. Its unique properties made it suitable for hydrophobic fabrication. The electrode was clamped in the quill, with the bottom surface facing to the top surface of the workpiece clamped on the vice, shown in Fig. 3.1.2. Both surfaces were aligned horizontally but in perpendicular directions; therefore, the textured surfaces would have a rectangular surface area in $15 \mathrm{~mm}$ x $12 \mathrm{~mm}$. The workpiece was prepared in small cubic shapes so that only one sample would be machined at a time. New milled electrode surfaces were used for new workpiece specimens to avoid topographic influence from the tool. To use a new tool every time might not be applicable for industrial uses; therefore, the effect of machining with the same electrode 
surface was also investigated which would be introduced in the next chapter. The polarity of the electrode was always set to be positive, and the workpiece to be negative. During the machining process, elemental carbon came from the dielectric oil would attach to the surface of the anode [39], so that the electrode surface could be protected by this carbon layer which significantly reduced the electrode wear. This carbon layer was also undesired on workpiece's surface because it would reduce the material remove rate.

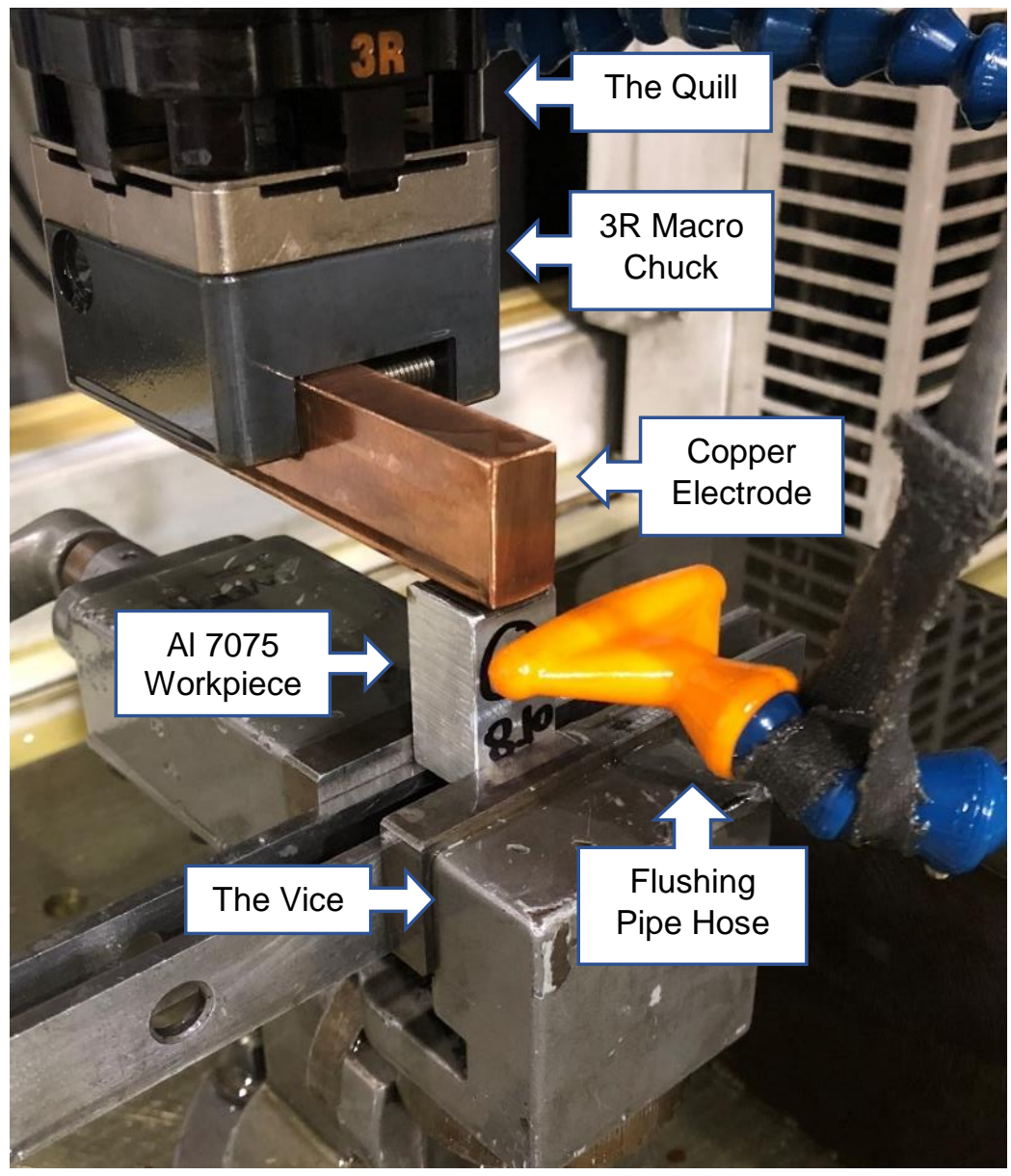

Fig. 3.1.2 Machining setup with the copper electrode clamped in the quill, and the Al 7075 workpiece clamped in the vice. 


\subsubsection{Grinding and Polishing}

A Struers Tegramin-25 Polisher was used for grinding and polishing process shown in Fig. 3.1.3 left. Specimens were prepared by mounting press with a diameter of $28 \mathrm{~mm}$. Six sample pieces could be placed onto the specimen holder of the polisher at the same time. The entire process included three steps: grinding, diamond paste polishing, and chemical polishing. Ultra fine surfaces could be obtained shown in Fig. 3.1.3 right.
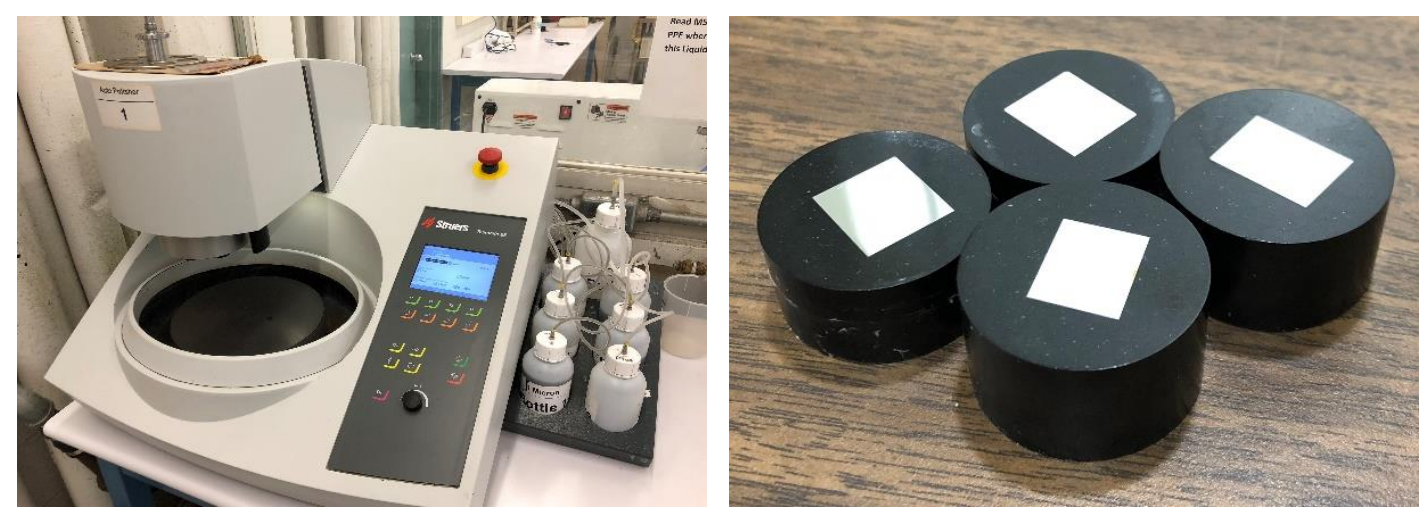

Fig. 3.1.3 Struers Tegramin-25 Polisher (left) and polished specimens (right).

Three different roughness levels of silicon carbide sandpapers were used for the grinding process, in an order of European standard \#500, \#1200, and \#4000. The workpiece surface was grinded for 90 seconds with each sandpaper by using water as the lubricant. A higher force of $15 \mathrm{~N}$ was provided for each sample, and the rotational speeds of the specimen holder and the sandpaper were $150 \mathrm{rpm}$ and $300 \mathrm{rpm}$ respectively in the same direction. The second step was to use diamond paste to polish the surfaces by using ethanol based lubricant. There were also three steps, which could polish the surfaces down to a 
mean roughness of $1 \mu \mathrm{m}$ in 10 minutes. A bigger force of $25 \mathrm{~N}$ was applied to all the specimens, and the rotational speeds were reduced to $150 \mathrm{rpm}$ for the polishing cloth and $120 \mathrm{rpm}$ for the holder in opposite directions. After this process, mirror-like surfaces could be obtained. The last step was a chemical polishing process, which used Oxide Polishing Suspensions (OP-S) to reduce the roughness level down to $0.05 \mu \mathrm{m}$ in two minutes. A force of $15 \mathrm{~N}$ and rotational speed of $90 \mathrm{rpm}$ were applied to the holder. The rotational speed of $150 \mathrm{rpm}$ was kept for the cloth in the same direction with the holder. Between every two steps, all the specimens were cleaned by using ethanol and then dried immediately by using a hair drier.

\subsubsection{Cleaning Methods}

After the machining process, a thin layer of black debris would sometimes remain on the surface of the workpiece depending on machining parameters which would be introduced more specifically in Chapter 4. Conventional cleaning solutions like acetone or ethanol could only remove a small amount of it. There were two methods that could fully clean the surfaces, the Ultrasonic Cleaner and a commercial spray called Electrical Wire Cleaner. Both two methods were examined by using clean surfaces generated by EDM process. Contact angles were recorded before and after the cleaning process. Ultrasonic cleaned surfaces indicated a decrease about $10^{\circ}$ to $15^{\circ}$, and the Electrical Wire Cleaner cleaned surfaces indicated an increase about $1^{\circ}$ which could be considered as the variation in measurement process. Furthermore, polished surfaces cleaned by ethanol were cleaned 
again by the Electrical Wire Cleaner to test if there was any influence in contact angle. No significant difference was observed before and after spraying the cleaner to the surfaces. Therefore, this cleaner was used as the cleaning process for all the experiments in this thesis.

\subsubsection{PDMS Casting}

PDMS casting process was conducted to study the opposite structure of EDM-textured surfaces. The mold preparation included three steps: mixture preparation, air bubble extraction, and oven heating. The silicone elastomer was mixed with a curing agent in a ratio of 10:1 in a small container. After the mixture was well stirred, a vacuum chamber with low pressure was used to accelerate the air bubble extraction. When no more bubbles arose from the mixture, the container was removed from the chamber, and the workpieces were carefully placed into the mixture. The surfaces being casted were placed parallel to the top surface of the liquid, so that the wettability analysis could be easier. The container was then placed into an oven and heated with a temperature around $100^{\circ} \mathrm{C}$ for 5 hours. A following cooling process was finally conducted until the container reached room temperature. After the entire process, a solidified transparent PDMS chunk, containing all the workpieces that were pre-placed, could be obtained. The chunk could be easily cut, and a PDMS casted surface could be obtained shown in Fig 3.1.4. 


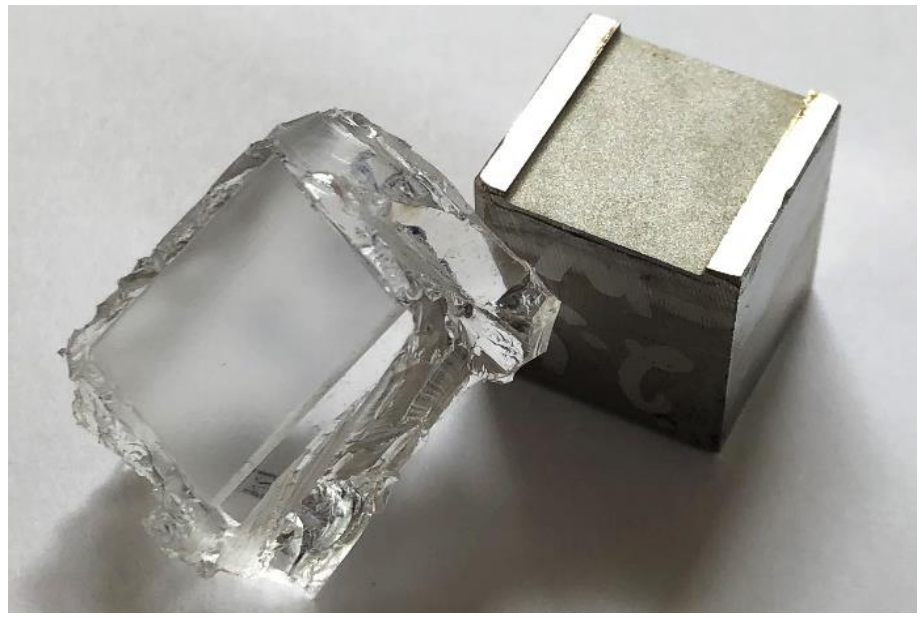

Fig. 3.1.4 An EDM textured surface with its PDMS casted surface.

\subsection{Wettability Analysis}

The High Speed Contact Angle Instrument OCA 35 is composed of five parts, a video camera, a motor-controlled stage, a Halogen light source, a liquid dosing system and a PC with the analytical software. It was briefly introduced in section 1.1 .2 , details will be explained more specifically in this section.

An USB-CCIR camera is used for the video system, with a maximum resolution of 768x576 pixels and maximum frame rate of 52 FPS. It contains a $6 \mathrm{X}$ parfocal zoom lens which can generate a $32 \mathrm{x}$ to $200 \mathrm{x}$ magnification. An integrated fine focus is adjustable so that high quality images can be extracted. The observation angle can also be modified to prevent hiding of the droplet from the surface edge. In front of the camera, there is a 100 $\mathrm{mm}$ X $100 \mathrm{~mm}$ sample stage that can support the sample with a maximum weight of three kilograms. The stage is traversable in a range of $100 \mathrm{~mm} * 100 \mathrm{~mm}^{*} 50 \mathrm{~mm}$ in $\mathrm{X}-, \mathrm{Y}-$, and 
Z-axis respectively. An auto controller is used for the movement of the stage, and the speed varies from $0.13 \mathrm{~mm} / \mathrm{s}$ to $3.95 \mathrm{~mm} / \mathrm{s}$. The accuracy of the stage positioning can reach \pm 0.01 $\mathrm{mm}$ in the sample plane and $\pm 0.005 \mathrm{~mm}$ in the vertical direction. On the other side of the stage, a halogen light source is used to help extract a clearer image of the droplet. The intensity of the light is controlled by either the software, or a rotating knob.

The electronic dosing system consists of multiple motor-driven syringes, syringe lines, needles, and controllers. Different syringes are used for different liquid to avoid contamination. A variety of customized needles with varied diameters are designed for different purposes. The position of the needles can be adjusted in a small range of distance to be aligned with the video system. The dispensing volume is controlled by the controller connected to the syringes. This can be accomplished manually by the operator, or automatically by the software. The minimum dosing amount can reach $0.05 \mu \mathrm{L}$ in a dosing rate from $0.06 \mu \mathrm{L} / \mathrm{s}$ to $26.4 \mu \mathrm{L} / \mathrm{s}$.

\subsubsection{Contact Angle Measurement}

The static contact angle was measured by using sessile drop method, which was the most accurate, repeatable and commonly used technique. The liquid selected in this experiment was Milli-Q water, which was a type of ultra-purified and deionized water.

Basically, the position of the sample surface was pre-adjusted to pick up the water droplet dispensed by the needle. The droplet could either be "picked up" by the surface, or drip by itself when the gravitational force overcame the drag force with the needle. If the 
surface had a high water-repellency, the former was not applicable; the latter method was used in the research. A needle diameter of $0.32 \mathrm{~mm}$ was selected so that a droplet with a volume about $6 \mu \mathrm{L}$ could fall on its own because of the gravity. The textured surfaces were moved to the height where the droplet just left the needle, shown in Fig.3.2.1, so that the kinetic energy could be minimized. Baseline detection was accomplished either automatically by the software, or manually by the operator. Then the static contact angle could be obtained, or a dynamic tracking mode could be activated so that the machine would automatically calculate the contact angles with a constant time step in a certain period. In this research, a drop size of $6 \mu \mathrm{L}$ was dispensed for each measurement. The static contact angle was recorded after 15 seconds to ensure that droplet reached the local equilibrium. Three surfaces were prepared for each type of texturing, and two measurements were constructed on each surface. Therefore, totally six measurements were taken for one specific kind of surfaces, and the average value was calculated along with the standard deviation.

Different fitting methods were introduced before in section 1.1.2. Ellipse fitting was the default calculating technique the machine would generate automatically. According to the literature [2], for the surfaces with a high hydrophobicity, ellipse fitting would be suitable with a droplet size smaller than $10 \mu \mathrm{L}$. Different contact angles were measured by using all four methods, and the results were compared with the manually measured results. Details will be discussed in Chapter 4. 


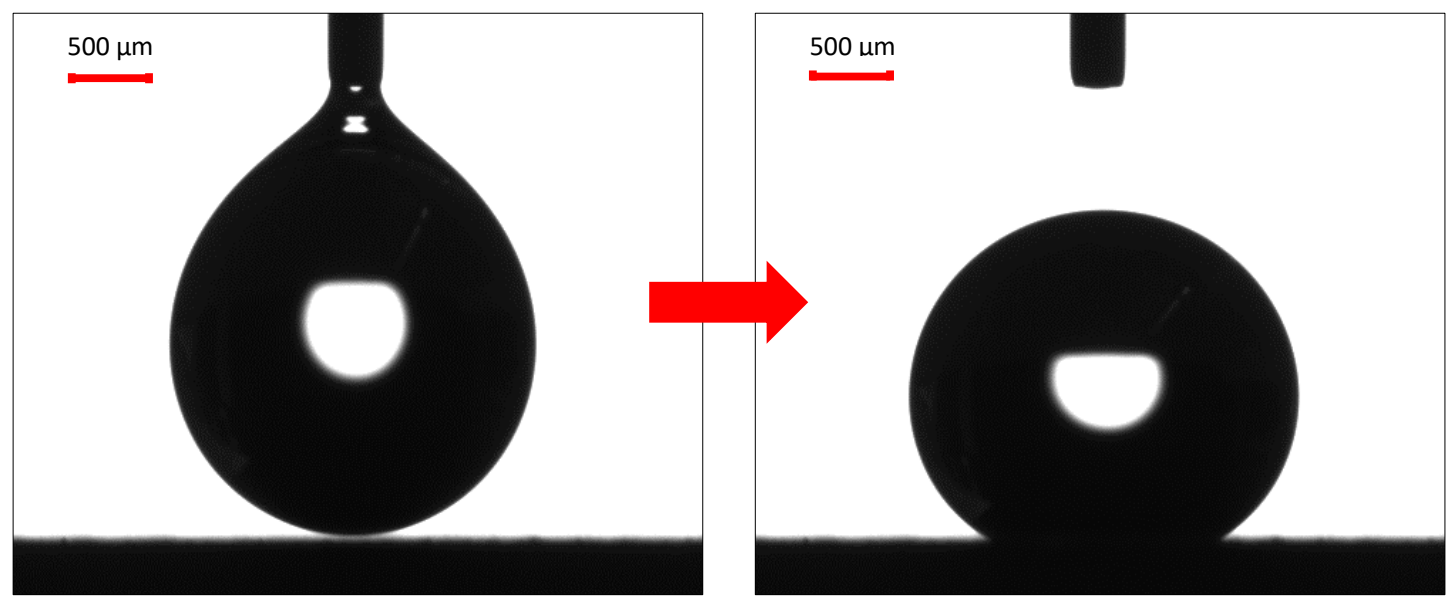

Fig. 3.2.1 Process of dispensing the droplet onto the textured surface.

\subsubsection{Contact Angle Hysteresis Measurement}

Wettability of surfaces consists of two aspects, static analysis which is the contact angle measurement, and dynamic analysis which represents the contact angle hysteresis measurement. The basic principle of CAH is the difference between the advancing contact angle and the receding contact angle when the droplet is moving with a certain velocity.

One of the most common methods to measure CAH is called Tilting Plate Method. Basically, the surface with a droplet is tilted until the droplet starts to slide. A high speed camera is used to capture the motion of the moving droplet, and the hysteresis can be calculated. Gravitational force is the only external force that is driving the droplet. Unfortunately, due to the limitation of the experimental setup, our stage did not carry a rotational motion. To imitate the external force given by the gravity, shown in Fig. 3.2.2, a 
needle remained in the water drop was used to drag the water drop by moving the stage. The same needle was selected to drive a $6 \mu \mathrm{L}$ water droplet, moving at a speed of $0.13 \mathrm{~mm} / \mathrm{s}$. The results will be discussed later in Chapter 4 .

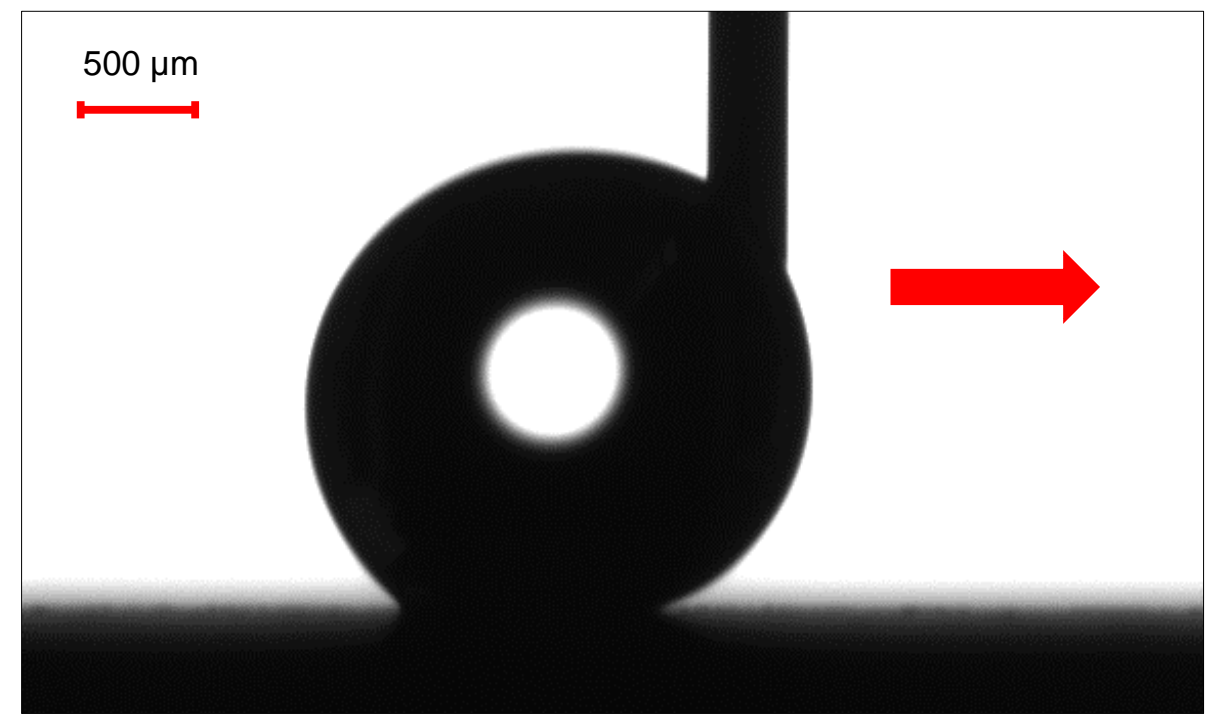

Fig. 3.2.2 Needle embedded dragging method to measure CAH moving with a speed of $0.13 \mathrm{~mm} / \mathrm{s}$.

\subsection{Surface Characterization}

\subsubsection{Confocal Microscope}

Alicona InfiniteFocus G5 is a white light interferometer that can analyze 3-Dimensional surface structures, with the resolution down to $9 \mathrm{~nm}$, without any contact. Varied magnifications from $2.5 \mathrm{X}$ to $100 \mathrm{X}$ can be selected with both LED Coaxial and high power Ringlight illumination. The maximum measurable slope can be as high as $87^{\circ}$, which is suitable for EDM machined surfaces that consists of sharp peaks and valleys. A built-in 
Vibration Absorption System helps to avoid surrounding disturbance, which makes the measuring process more stable.

$1 \mathrm{~mm}$ X $10 \mathrm{~mm}$ area of textured surfaces was scanned for each analysis. After the form removal, the arithmetic average roughness $(\mathrm{Ra})$ and arithmetic mean height $(\mathrm{Sa})$ were calculated as a reference for the surface roughness levels. More than seven thousand coordinates were extracted through a $10 \mathrm{~mm}$ straight line scanned on the surface, in a step size of $1.4 \mu \mathrm{m}$ in $\mathrm{x}$-axes. Then the coordinates were inputted into MatLab to construct the surface profile, as well as the Fast Fourier Transform (FFT).

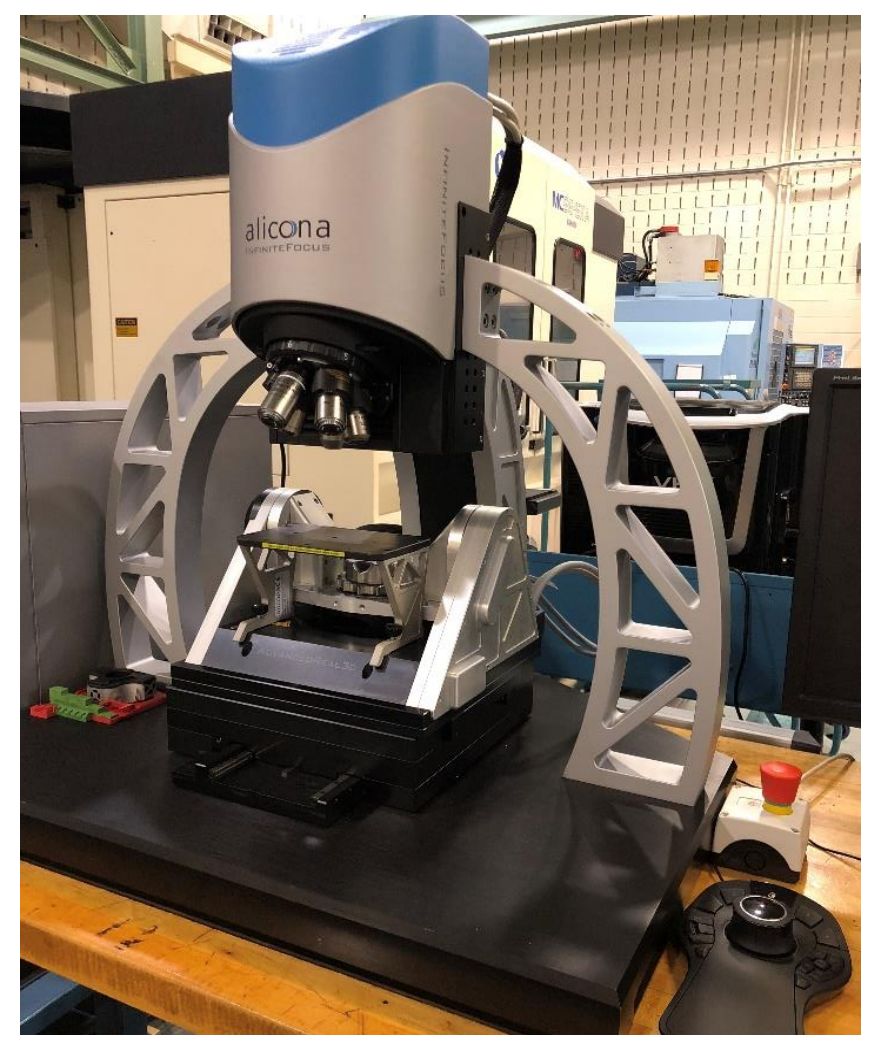

Fig. 3.3.1 Alicona InfiniteFocus G5 White Light Interferometer. 


\subsubsection{Scanning Electron Microscope (SEM)}

A model JSM-6610LV SEM, located in the Canadian Centre for Electron Microscopy (CCEM) at McMaster, was used to observe the surface details. The magnification can reach up to $300,000 \mathrm{X}$ with the highest resolution down to $3 \mathrm{~nm}$. The automatic focusing and brightness adjusting functions can help generate extremely high quality images. A build-in Energy Dispersive X-ray Analyzer (EDX), refer to as EDS, provides an elemental analysis of sample pieces.

An accelerating voltage of $10 \mathrm{kV}$ was used for both SEM and EDS analysis. Images with the magnification ranged from $300 \mathrm{X}$ to $2000 \mathrm{X}$ were taken to observe the surface structures. Elemental analysis was conducted in points and areas to compare the chemical composition for different surfaces. This was mainly used to investigate the difference among surfaces machined in different dielectric mediums, as well as the surfaces stored for varied length of periods.

\subsubsection{High Speed Camera}

A Photron Fastcam SA-Z High Speed Camera was used to observe the behaviour of impacting droplets. The maximum frame rate can go up to 2.1 million frames per second (fps). With a frame rate lower than $20,000 \mathrm{fps}$, a high resolution of $1024 * 1024$ pixels can be obtained. Three LED lights in different sizes and brightness levels are prepared upon different requirements. 
In this thesis, a frame rate of $6,000 \mathrm{fps}$ was used to observe the motion of impacting droplets on the surfaces with high hydrophobicity. Droplets with a volume of $10 \mu \mathrm{L}$ were dispensed $5 \mathrm{~cm}$ above the surfaces. The brightest LED light was selected so that distinct videos could be obtained. Picture series were directly obtained from the camera, and the software ImageJ was used to generate videos. 


\section{Chapter 4}

\section{Results and Discussion}

In this chapter, in terms of fabricating superhydrophobic surfaces by using Sink EDM, a proof of concept is demonstrated with the explanations of the science behind it. The factors influencing wettability including machining parameters, dielectric mediums, surface structures, surface chemistries and workpiece materials are analyzed and discussed. Surface profiles, Fast Fourier transform of surface profiles, surface chemical compositions generated by using surface characterization technologies are also provided for further explanations.

\subsection{Proof of Concept}

Young's contact angle depends on the surface free energy of the material, so it is also called the intrinsic contact angle. In this study, the material used predominantly was Aluminum 7075 alloy. Several samples were polished for the measurement of the Young's contact angle. Flat surfaces with an arithmetical mean roughness (Ra) of $0.05 \mu \mathrm{m}$ were obtained, and a contact angle of $59.4^{\circ} \pm 1.64^{\circ}$ was measured (Fig. 4.1.1 (a) and (b)). After Sink EDM texturing, the contact angle was increased to $149.4^{\circ} \pm 0.77^{\circ}$ with a CAH of $15.3^{\circ} \pm 2.62^{\circ}$ which indicated high hydrophobicity shown in Fig. 4.1.1 (c) and (d). 


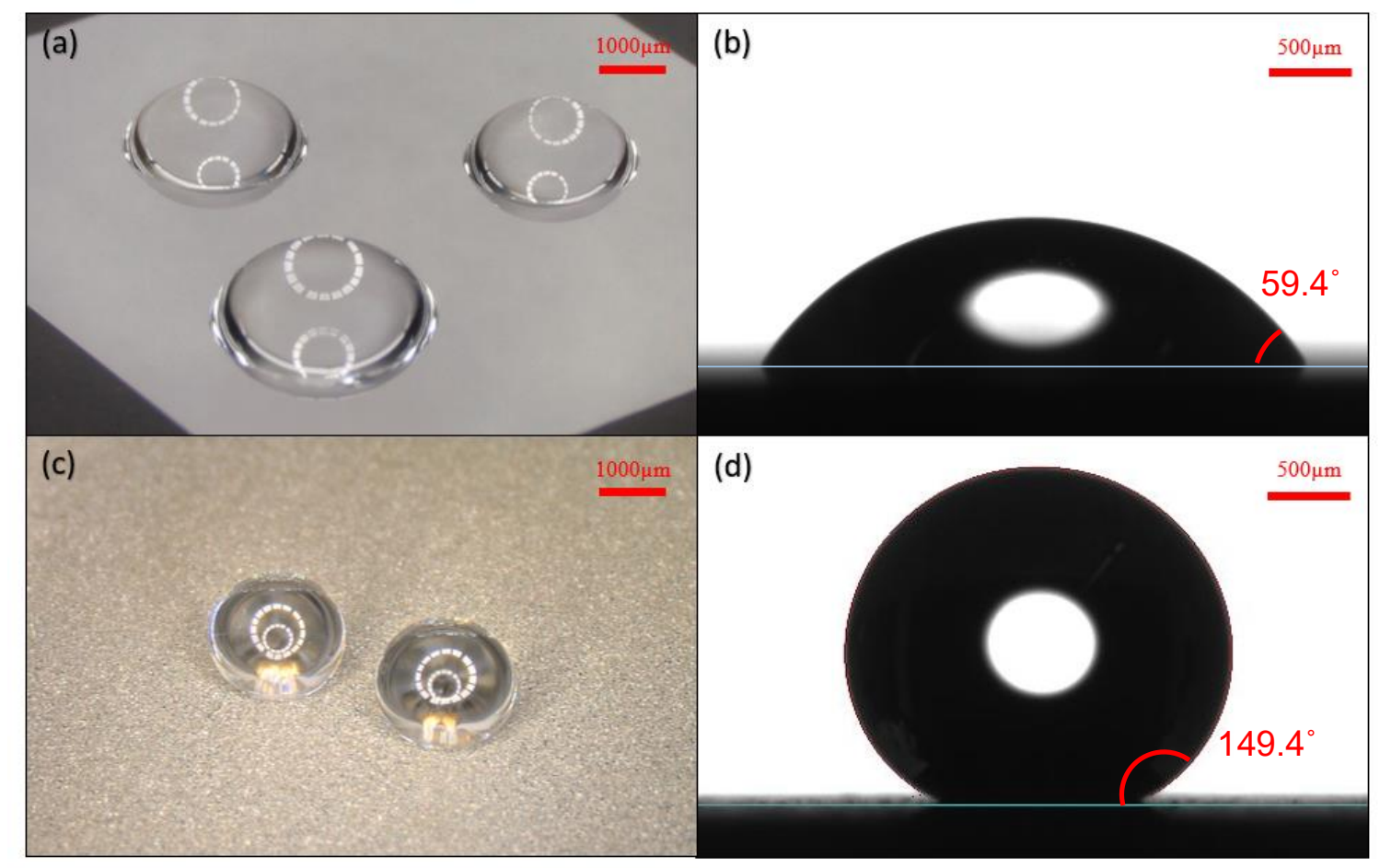

Figure 4.1.1 Water droplets on a polished surface and a Sink EDM textured surface taken by (a, c) regular microscopy and (b, d) goniometer respectively.

Surface resistance of the water impact was investigated by taking slow motion video of a droplet falling onto different surfaces. Water droplets were released $5 \mathrm{~cm}$ above the surfaces, and a sequence of pictures was captured by using the High Speed Camera at a speed of 6000 frames per second for every experiment, as shown in Fig. 4.1.2. On a polished sample, the water drop adhered to the surface on contact; however, on an EDM textured surface, the water drop bounced back on the surface which indicated a completely non-wetting behavior. The textured surface was tilted at an angle of 15 degrees. The water droplet bounced several times until it was cleared off the surface, as shown in Fig. 4.1.3. 
These experiments demonstrated the superhydrophobicity of the Sink EDM textured surfaces, as well as the high resistance of the structure to water impact.

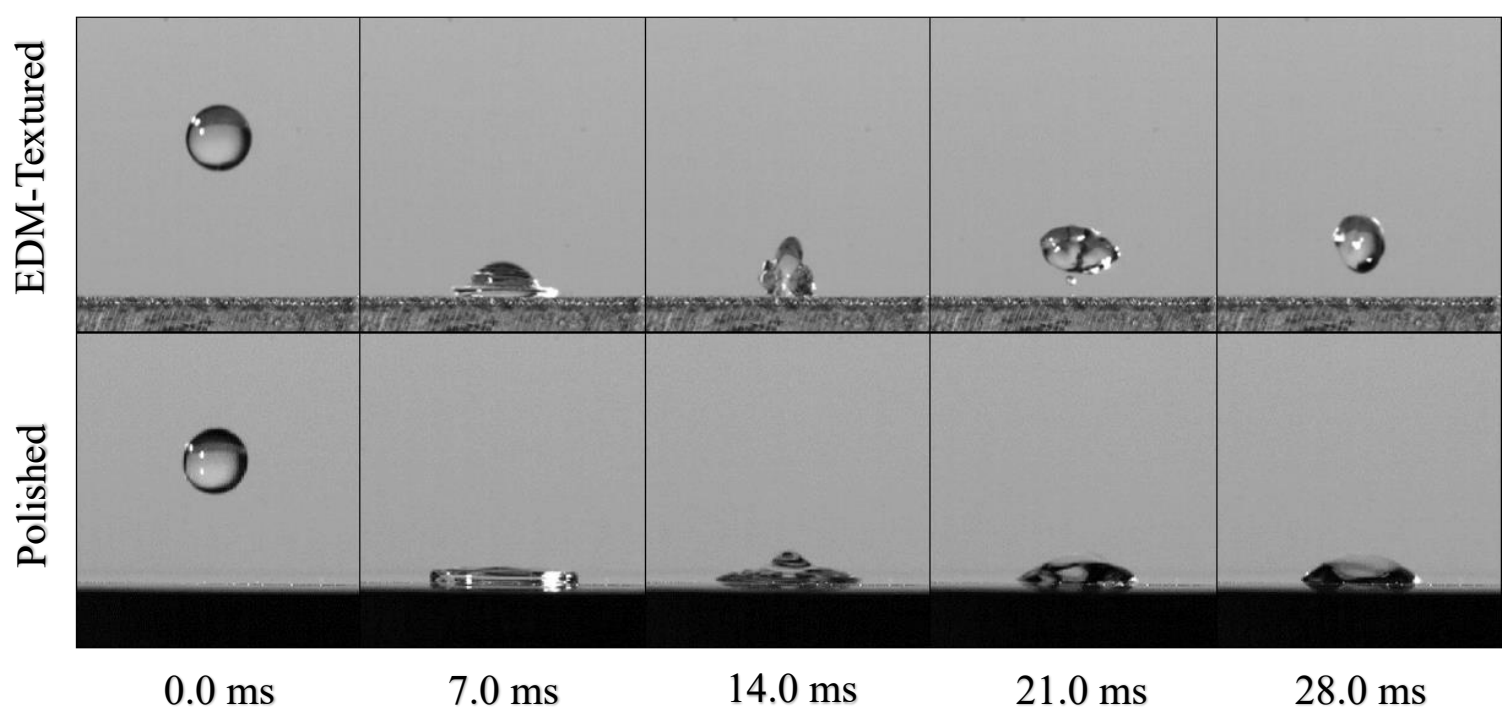

Fig. 4.1.2 Picture sequences of an impacting water droplet.
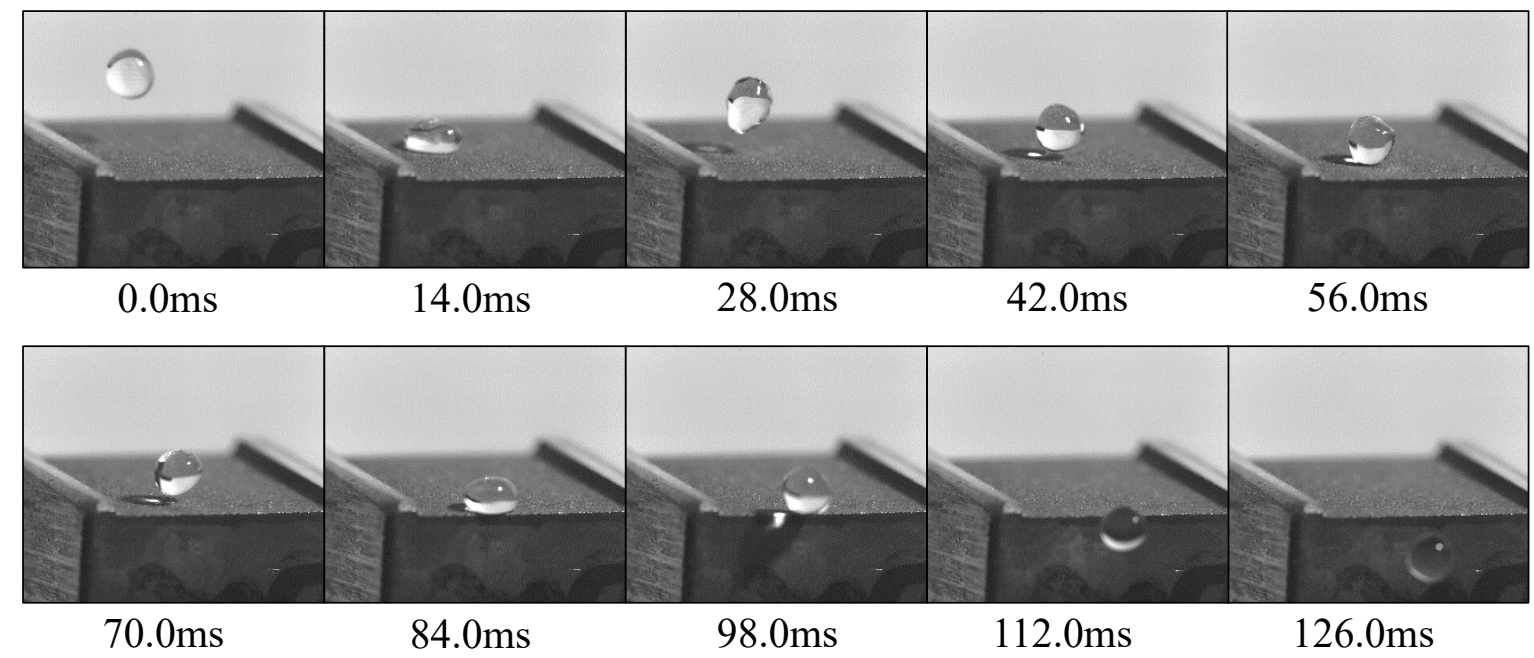

Fig. 4.1.3 Picture sequence of an impacting water drop on an EDM textured surface tilted at $15^{\circ}$. 


\subsection{Contact Angle Measurement}

Four different contact angle fitting methods were introduced in chapter 1, a comparison of which is analyzed in this section. All the contact angle measurements, automatically generated by the software, were compared with the results calculated manually. The manual process was accomplished by using the software ImageJ, a Java-based scientific image processing program, to calculate the contact angles from images of the water drop extracted from the goniometer. Every profile was measured six times and the average value was used for the analysis.

Three different contact angles in hydrophobic regime were selected for the investigation, as shown in Fig. 4.2.1. The manually calculated values were $115.8^{\circ} \pm 1.64^{\circ}$, $136.0^{\circ} \pm 1.93^{\circ}$ and $151.9^{\circ} \pm 0.77^{\circ}$ respectively. On the other hand, these same images were input into the software SCA 20 to generate contact angles by using four fitting methods automatically. All the results are shown in Table 4.1, and (a), (b), (c) are corresponding to the letters shown in Fig. 4.2.1. The results calculated by using ImageJ were assumed to be the reference values.
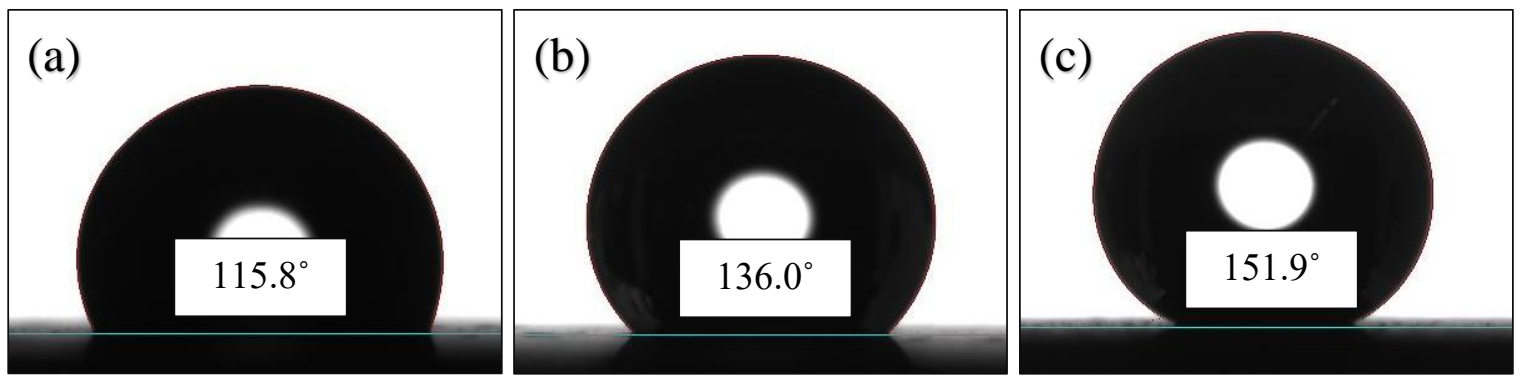

Fig 4.2.1 Manual contact angle measurements on three surfaces. 
Table 4.1 Contact Angles Generated by Four Fitting Methods.

\begin{tabular}{|c|c|c|c|c|}
\hline & Circle Fitting & Tangential Fitting & Ellipse Fitting & Y-L Fitting \\
\hline (a) & $111.3^{\circ}$ & $108.7^{\circ}$ & $115.4^{\circ}$ & $117.4^{\circ}$ \\
\hline (b) & $129.8^{\circ}$ & $128.4^{\circ}$ & $135.0^{\circ}$ & $140.7^{\circ}$ \\
\hline (c) & $142.3^{\circ}$ & $142.5^{\circ}$ & $149.4^{\circ}$ & $167.3^{\circ}$ \\
\hline
\end{tabular}

Circle fitting and tangential fitting methods indicate large errors, which is supported by the literature [2]. With the increase of surface hydrophobicity and the drop volume, the droplet tends to be distorted more by the gravitational force. To assume a circular shape for the droplet will underestimate the real contact angle value. Tangential fitting method directly generates the tangential line for the droplet from the three-phase contact interface; however, it can be easily disturbed by the contaminants on the surface or even the roughness.

The conventional methods of measurement are ellipse fitting and Young-Laplace (Y-L) fitting methods. Y-L fitting technology, which is also called axisymmetric drop shape analysis (ADSD), is the most widely used method for large contact angle measurements by most of the researchers. Ideally, Y-L fitting method was supposed to be used in this thesis; however, the contact angles generated by such technique seemed to overestimate the results especially for case (c). Contact angle of $167^{\circ}$ was about $15^{\circ}$ higher than the contact angle measured manually. With respect to other methods, ellipse fitting revealed a higher accuracy. The difference with the reference values was larger when the contact angle became higher, from $0.4^{\circ}$ for (a) to $2.5^{\circ}$ for (c), which was a variability in the 
measuring process. Due to the gravitational force, the three-phase intersection could hardly be recognized when a water drop was resting on a surface with high hydrophobicity. This might be the reason why ellipse fitting gave smaller error with lower contact angles.

The same situation has also been observed by our researchers. Zhang et al. [40] made a comparison among all four fitting techniques shown in Fig. 4.2.2. The blue line along the droplet profile was automatically generated by four fitting methods to calculate their relative contact angles. The three-phase contact point was not correctly detected by using circle fitting (b) and tangential fitting (c), which led to an underestimation of the contact angle. The Y-L fitting indicated an extremely high contact angle of $179.8^{\circ}$. This meant that the surface was almost perfectly non-wetting, which was not realistic when other methods calculated contact angle values between $150^{\circ}$ to $160^{\circ}$. The profile line did not go along the droplet profile which might be the reason for obtaining the high contact angle. Compared to all other methods, the extracted profile of ellipse fitting seemed more reasonable and accurate in this case. The extracted profile line did not fit well with the real profile of the water drop; however, the three-phase intersection was located precisely which helped to enhance the calculation accuracy.

In conclusion, most of the surfaces generated in this thesis were hydrophobic. Manual calculation of the contact angles was not used to avoid bias. The ellipse fitting method, which indicated less error, was selected to be the calculation technique for all the measurements. 

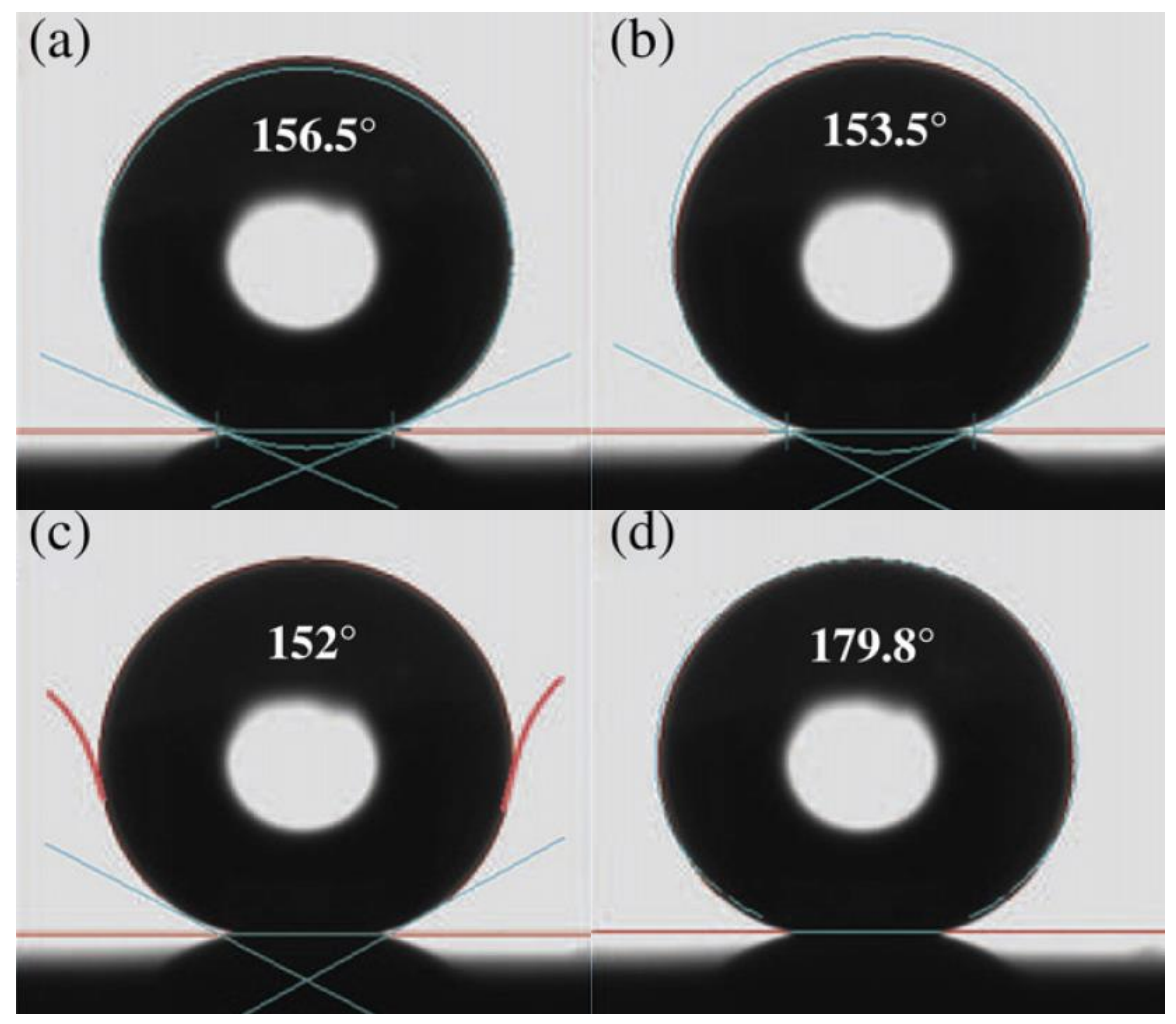

Fig. 4.2.2 Four fitting methods: (a) Ellipse fitting, (b) Circle fitting, (c) Tangential fitting, and (d) Y-L fitting use to calculate the static contact angle of a sessile droplet [40].

\subsection{Machining Variables and Hydrophobicity}

\subsubsection{Machining Parameters}

The first part of this phase of experiments was to discover the influence of discharge energy on the contact angle. In EDM process, surface structure of workpieces is determined by the 
spark energy applied by the machine. This energy, E, is product of discharge voltage U, discharge current I, and the discharge duration T:

$$
\mathrm{E}=\mathrm{U} * \mathrm{I} * \mathrm{~T}
$$

The discharge voltage is usually kept constant around $20 \mathrm{~V}$ controlled by the machine; therefore, this section focuses on the wettability change by varying the current and the discharge duration. A higher current increases the current density and the temperature of the arc column, which helps generate deeper craters on the surfaces. A higher discharge duration increases the diameter of the plasma channel, which decreases the energy density. This results in shallower but wider craters on the surfaces. Pulse duration includes two parts: ignition delay and discharge duration. Ignition delay depends on the gap condition, which means it can be different for every discharge cycle. Therefore, discharges were set to correspond to the discharge duration, so that the craters can be more uniform.

To machine aluminum alloys by using copper electrodes, the ontime should be numerically several times bigger than the current values. This could help generate a stable machining process and enhance the machining speed. Excessively large ontime-to-current ratio could significantly reduce the material removal rate. To the contrary, a high currentto-ontime ratio could cause arcing, which could damage the electrical circuits and the power supply of the machine. However, for research purposes, extreme conditions were 
attempted in this group of machining. Five different current values were selected, $6.2 \mathrm{~A}, 17$ A, 29 A, 39 A, 52 A, which almost covered the entire range that the machine could generate. Three groups of experiments were performed with discharge duration, $6.5 \mu \mathrm{s}, 18 \mu \mathrm{s}$, and $56 \mu \mathrm{s}$.

As the machining process proceeds, the roughness of the electrode surface increases due to wear. After a certain amount of machining, this roughness value converges to a constant range depending on the spark energy. The electrode roughness can directly affect the surface finish of the workpiece [41]. Therefore, in different experiments, the best way to reduce the variability in the workpiece surface is to converge the electrode surface to the same roughness range. This range varied with different machining parameters, so a machining depth into the workpiece of $800 \mu \mathrm{m}$ was implemented, which sacrificed machining time for stability. The parameters selected for these groups of machining generated rough surfaces, with the Ra value ranged from $1.4 \mu \mathrm{m}$ to $8.0 \mu \mathrm{m}$; therefore, they were called primary texturing or roughing. Corresponding contact angle results are shown on the left column in Fig. 4.3.1.

No significant difference was observed statistically but a slight decreasing trend was applied to all three curves. As the crater size increased, droplets could penetrate the surface structure more easily which led to a slight decrease in the observed contact angles. Contact angles were usually measured six times on the same surface so that the average value and deviation could be calculated. In this research, six measurements were taken on three surfaces machined by using same parameters. As a result, the error bar also included 
the structural variability from different machining processes. However, the results did not indicate large variabilities, which illustrated the stability of the EDM machining process.

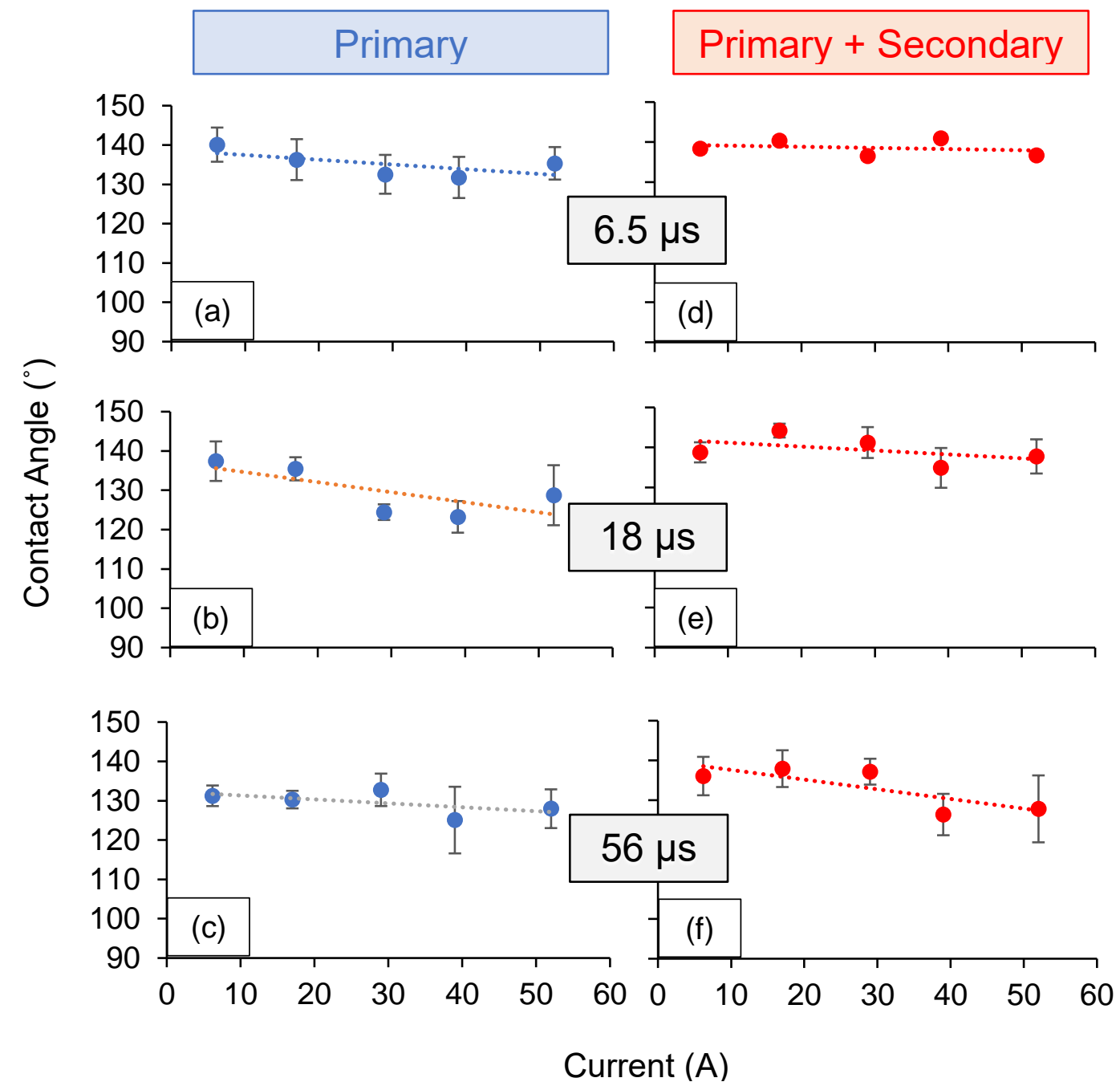

Fig. 4.3.1 Contact angle Vs. Current in 3 groups of discharge durations values with (a), (b), (c) primary texturing only and (d), (e), (f) primary texturing plus secondary texturing.

Before further discussing the results shown above, it is worth introducing the pillar array designs investigated by many researchers [42-45] first. Fig. 4.3 .2 illustrates some 
examples of such surfaces. Pillars with different shapes were studied by Yeo et al. [44], and they came up with two equations which fitted all the shapes in both Wenzel state and Cassie-Baxter state:

$$
\begin{aligned}
& \text { W. state: } \\
& \qquad \cos \theta^{W}{ }_{e}=\left(1+\frac{P H}{(a+d)^{2}}\right) \cos \theta_{e} \\
& \text { C-B. state: } \quad \cos \theta^{C}{ }_{e}=-1+\frac{A}{(a+d)^{2}}\left(1+\cos \theta_{e}\right)
\end{aligned}
$$

where $a, d, A, H, P$ were pillar width, spacing, top area, height and perimeter of the top area respectively. To maximize the Wenzel contact angles, pillar width and the spacing were desired to be smaller, which made the pillars thin but dense. On the contrary, pillar width and the spacing were desired to be bigger for Cassie Baxter contact angles, and the pillar top surface area to be smaller. These changes could both reduce the contact between the surface and the droplet.

The transition from Cassie Baxter state to Wenzel state could not be visualized directly from the equations. The density and thickness of the pillars decided if the droplet could penetrate the gaps. Varanasi et al. [43] concluded the influence in contact angles and wetting states regarding the spacing-to-width ratio shown in Fig. 4.3.3. The sessile droplets transitioned from Cassie Baxter state to Wenzel state after the ratio exceeded 6. Contact angles dropped about $40^{\circ}$ due to this transition, and the standard deviation increased 
significantly. The phenomenon indicated that an excessive spacing or deficient pillar thickness could lead to Wenzel State, reduce the contact angle, and increase the variability.

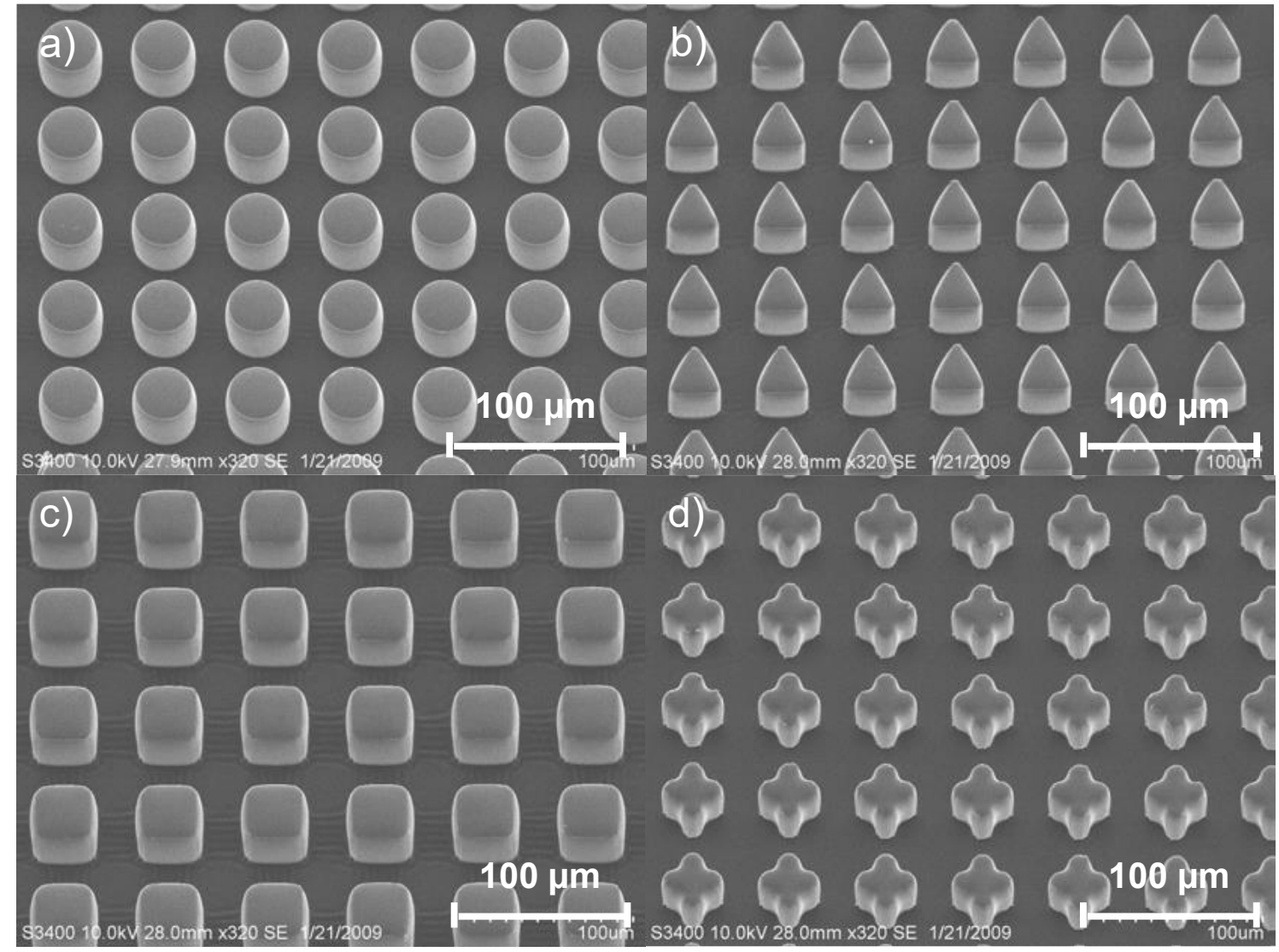

Figure 4.3.2 Fabrication of pillar array designs with a) circular, b) triangular, c) square, and d) cross-shape top area to investigate the hydrophobicity [44].

This conclusion agrees well with (a), (b), and (c) in Fig. 4.3.1. EDM machined surfaces consisted of peaks and valleys, which played the same role as pillars and spacing areas. With smaller parameters, the spacing-to-width ratio was smaller, resulting in higher contact angles in Cassie Baxter state. On the other hand, rougher surfaces indicated higher 
variabilities than finer surfaces, which might due to two reasons. The first one was that the roughness scale was too large for the droplets, so that the droplets were distorted. Different spots of the surface shaped the droplet differently, which could cause a large variance of the measurements. The second reason was that the droplets transited from Cassie Baxter state to Wenzel state. In Fig. 4.3.3, the last two measurements located at Wenzel regime also indicated much larger errors than the contact angle sitting in Cassie Baxter state.

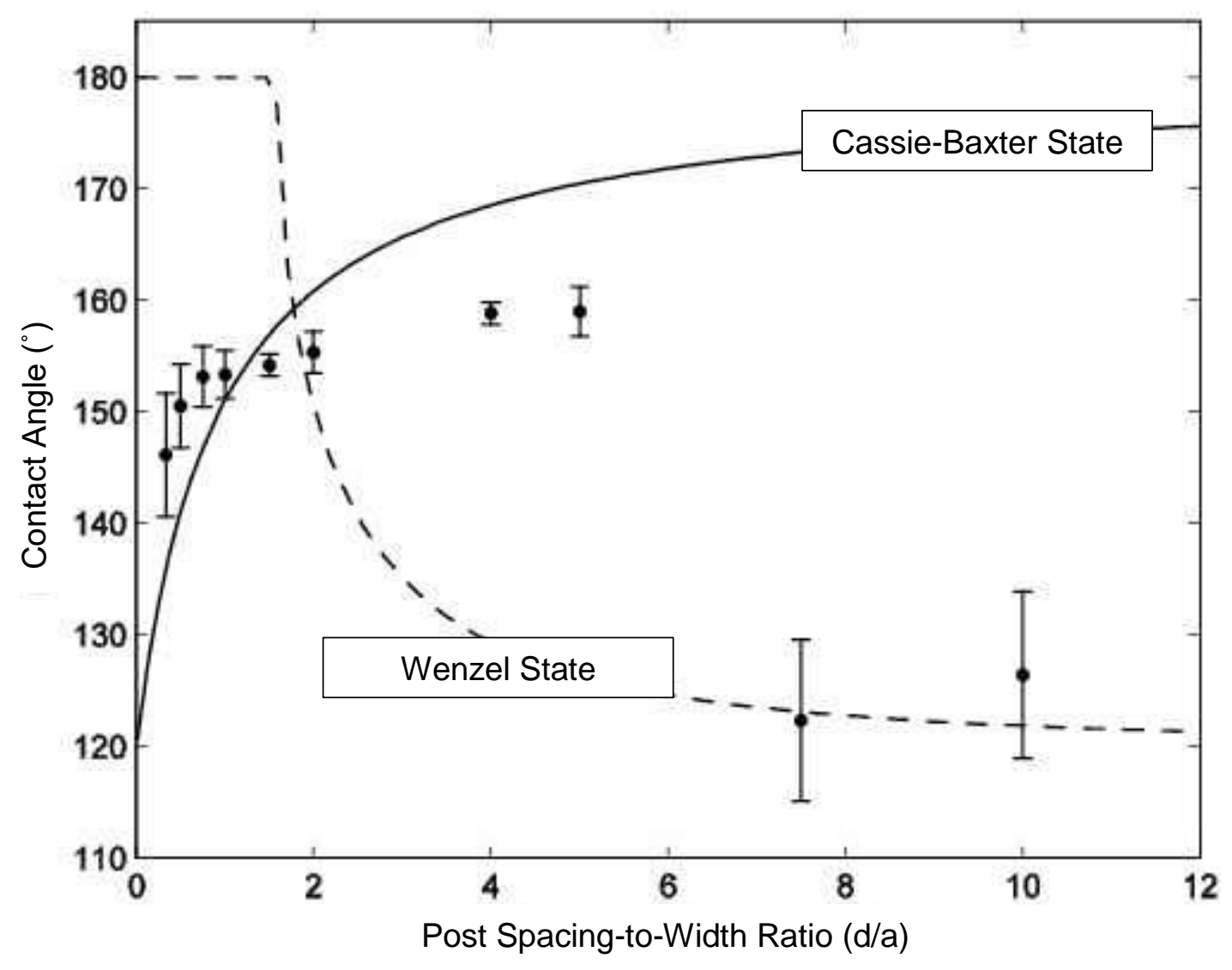

Fig. 4.3.3 Contact angle Vs. pillar spacing-to-width ratio with the analysis of Cassie Baxter State and Wenzel State [43]. 
As mentioned in the first chapter, fabrication of a hierarchical structure is desired for surfaces to obtain a high water repellency. Therefore, a secondary texturing is needed on the top of the primary texturing. The smallest parameters the machine tool can generate are 1.2 A for the current and $0.4 \mu$ s for the discharge duration. This group of parameters were used as the secondary texturing or finishing in the entire thesis. A texturing on the top of another one could hardly be controlled in the term of depth; as a result, machining time was monitored instead.

On the top of the primary texturing, a finishing process was constructed with a machining time of five seconds. Results are shown in Fig 4.3.1 (d), (e), and (f). Compared to the results obtained from primary texturing only, variability was reduced especially for the groups with discharge duration $6.5 \mu \mathrm{s}$. In the graph (d), the standard deviation was reduced significantly to $2.5^{\circ}$ after the finishing process, which meant that the secondary texturing made the surfaces more uniform and homogenous. Similar effect could also be observed in graph (e) comparing to (b). However, the reduction in deviation was not as significant as the comparison between (a) and (d). This was because that the surfaces generated in (b) were much rougher compared to (a). Five seconds of the finishing process could not remove enough material; therefore, the surfaces in (e) were not as uniform as surfaces in (d) which caused a higher variability in (e). This also explained why there was almost no change in the variability after a finishing process on the top of the roughest primary texturing (group (c) to (f)). In terms of contact angle, the trend lines were flattened for the first two groups after the finishing process. The second group, from (b) to (e), indicated the most significant improvement. This was because the finest group with 
discharge duration $6.5 \mu \mathrm{s}$ did not change significantly after the finishing process, and the roughness group needed longer finishing time to conduct changes. Only surfaces with a discharge duration of $18 \mu$ s experienced a big change in structures. The other possibility might due to the changes in wetting state regarding the spacing-to-width ratio. The finishing process removed the sharp peaks generated by roughing process, which reduced the spacing-to-width ratio at the same time. The pillars were already very dense for the groups with discharge duration of $6.5 \mu \mathrm{s}$, therefore the droplets were evidently already in Cassie Baxter state. On the other hand, for the surfaces machined by using discharge duration 56 $\mu \mathrm{s}$, five seconds of finishing process were insufficient so that Wenzel state could be applied to both graph (c) and (f). The transition only happened from graph (b) to (e) which caused the significant increase in contact angles. This possibility was not proved, but the explanation fitted well with the results; therefore, it might be worth for some future work.

Following on the previous experiment, the influence of secondary texturing machining time was studied shown in Fig. 4.3.4. Three groups of surfaces with different roughness levels were selected. The machining parameters, shown on the bottom right corner of each graph, represented the primary texturing parameters of relative surfaces. The secondary machining time covered a time span of 35 seconds, where $0 \mathrm{~s}$ meant that the surfaces had primary texturing only. 


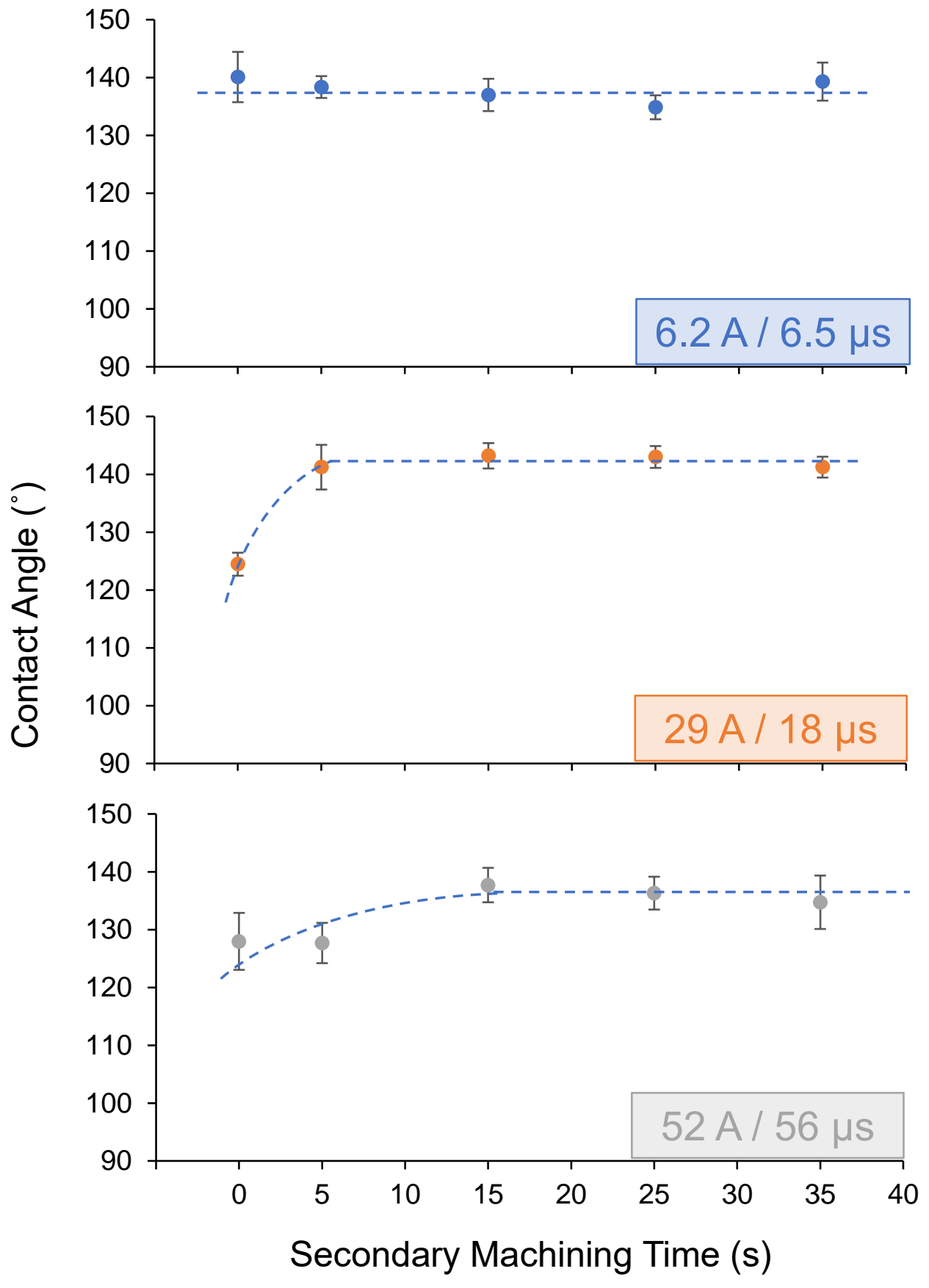

Fig. 4.3.4 Contact angle Vs. Secondary machining time with different groups of primary texturing parameters. 
In the first graph, with the smallest primary texturing parameters, no significant changes were observed by varying the machining time of secondary texturing. Similar results were obtained over the entire range, which proved that the secondary texturing could hardly change the surface structure machined with the finest primary texturing parameters. The second graph, machined by using $29 \mathrm{~A}$ and $18 \mu$ s for primary texturing, indicated a jump at five seconds. The contact angle increased approximately $15^{\circ}$ after five seconds of finishing process. The most interesting part was the enhancement shown in the third graph. Contact angle remained the same after 5 seconds of machining but increased after 15 seconds. This phenomenon proved the conclusion made in the previous experiment, that the surfaces with higher roughness needed a longer secondary machining time for contact angle enhancement.

To obtained surfaces with high contact angles, the machining parameters of $6.2 \mathrm{~A}$ and $6.5 \mu$ s seemed to give consistent values regardless of finishing time. Only roughing was needed to fabricate surfaces with contact angles around $140^{\circ}$. However, to machine a depth of $800 \mu \mathrm{m}$ for a surface area of $0.96 \mathrm{~mm}^{2}$, at least 40 minutes were needed which consumed a great amount of time and energy. The second group with roughing parameters of $29 \mathrm{~A}$ and $18 \mu \mathrm{s}$ might be more suitable in terms of machining time. The fabrication process only needed five minutes of roughing and five seconds of finishing. The last group shown in Fig. 4.3.4 could also generate surfaces with high contact angles rapidly. However, the energy consumption was also high due to large parameters. A machining time of two minutes might not be worthy comparing to the second group. In addition, the highest contact angle was about five degrees lower which could be another consideration. 
On the other hand, after the first jump in contact angles, no increasing trend was observed with a longer machining time for the last two graphs in Fig. 4.3.4. A further secondary machining would eventually interfere with the structure generated by the primary texturing, so that only an extremely fine surface would remain. To keep reducing the roughness would push the surface to a polished condition, which might lower the contact angle. To this end, an experiment was conducted by varying discharge duration with a fixed current of $6.2 \mathrm{~A}$. The smallest current value was selected from the previous machining so that finer surfaces could be obtained. Several groups of results obtained from the previous experiment were directly used. Seven discharge durations were selected in this experiment: $2.1 \mu \mathrm{s}, 3.2 \mu \mathrm{s}, 6.5 \mu \mathrm{s}, 18 \mu \mathrm{s}, 32 \mu \mathrm{s}, 42 \mu \mathrm{s}$, and $56 \mu \mathrm{s}$, which covered a wide range of roughness levels that 6.2 A of current could generate. Results are shown in Fig. 4.3.5.

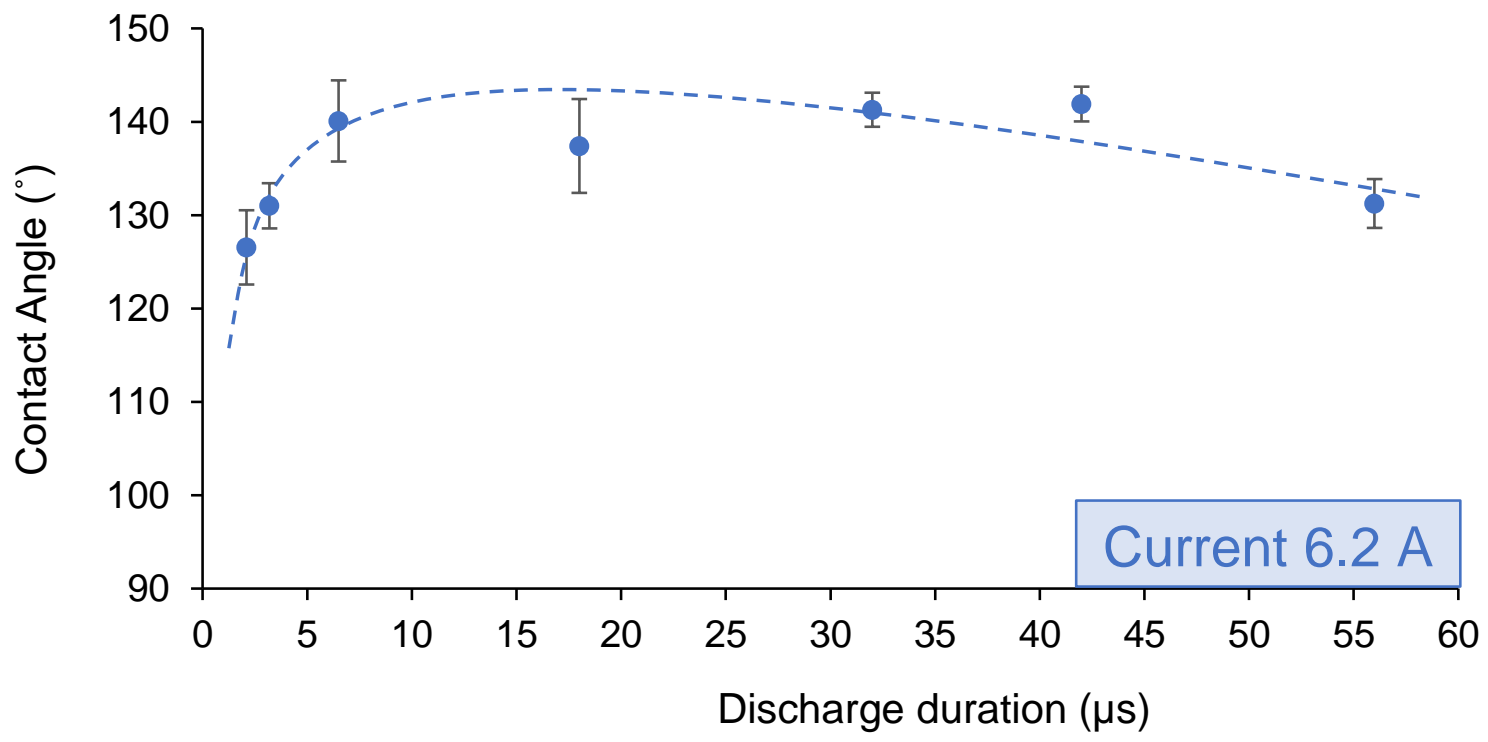

Fig. 4.3.5 Contact angle Vs. discharge duration with a current of 6.2 A. 
The highest contact angle appeared between $5 \mu$ s to $10 \mu$ s. Higher discharge duration could cause a decrease of contact angle which was already explained before. Lower discharge duration also reduced the contact angle due to low roughness levels. Further decrease of the roughness would push the surface towards a polished condition. A contact angle of $59.4^{\circ}$ was measured on a polished surface with a mean roughness of 0.05 $\mu \mathrm{m}$ introduced at the beginning of this chapter. If EDM was assumed to be able to fabricate surfaces with same roughness, the trend line of Fig. 4.3.5 would still not intersect y-axis at $59.4^{\circ}$. This was because of the change in surface chemistry after EDM process.

Surfaces with lower roughness were desired to be machined in terms of Ra value; however, the process could not proceed due to machining issues. In EDM machining process, extremely small parameters would generate ultra-small debris particles which could deposit onto the workpiece surfaces shown in Fig. 4.3.6. Machining process could not proceed when this black carbon deposition was generated. Instead of removing materials from the workpiece, deposition layers would be built up gradually. This layer could be hardly cleaned by spraying ethanol based solutions. Even the Electrical Wire Cleaner, or the Ultrasonic Cleaner could only remove a small amount of it. Ultra small parameters could be only used on the top of rough surfaces due to the low material removal rate. 


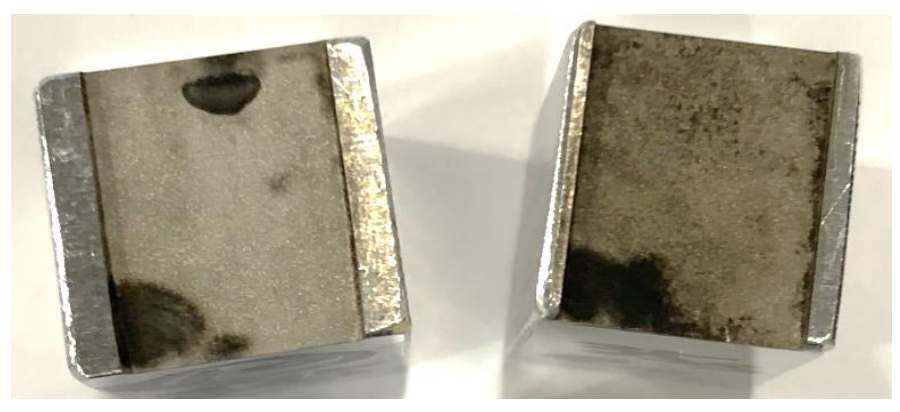

Fig. 4.3.6 Aluminum alloy surfaces, machined with small parameters, generated black depositions.

\subsubsection{Surface Structural Characterization}

To investigate the structural difference between surfaces with primary texturing only and with both primary and secondary texturing, a pair of surfaces with the same primary texturing parameters were selected from both groups. Surface profiles were extracted for both surfaces shown in Fig. 4.3.7. In this experiment, the primary texturing parameters of the selected surfaces were $17 \mathrm{~A}$ for the current and $18 \mu$ s for the discharge duration. Contact angle increased about $10^{\circ}$ after five seconds of secondary texturing, and the standard deviation became almost invisible. Data points can be found in Fig. 4.3.1 (b) and (e).

Compared to profile (a) in Fig. 4.3.7, the surface peak-to-valley distance in (b) was shortened because of the secondary texturing, from approximately $45 \mu \mathrm{m}$ to $30 \mu \mathrm{m}$. Sharp peaks were cut off which made the surface more uniform. Fast Fourier Transform (FFT) was conducted for further analysis, shown in Fig. 4.3.8. Compared to the surface with primary texturing only, the FFT for the second graph indicated a decrease in the amplitude without a sign of another frequency peak. This result implied that the finishing process did 
not create a hierarchical structure. The secondary texturing only removed peaks generated by the primary texturing, and the surfaces became more uniform and homogeneous.

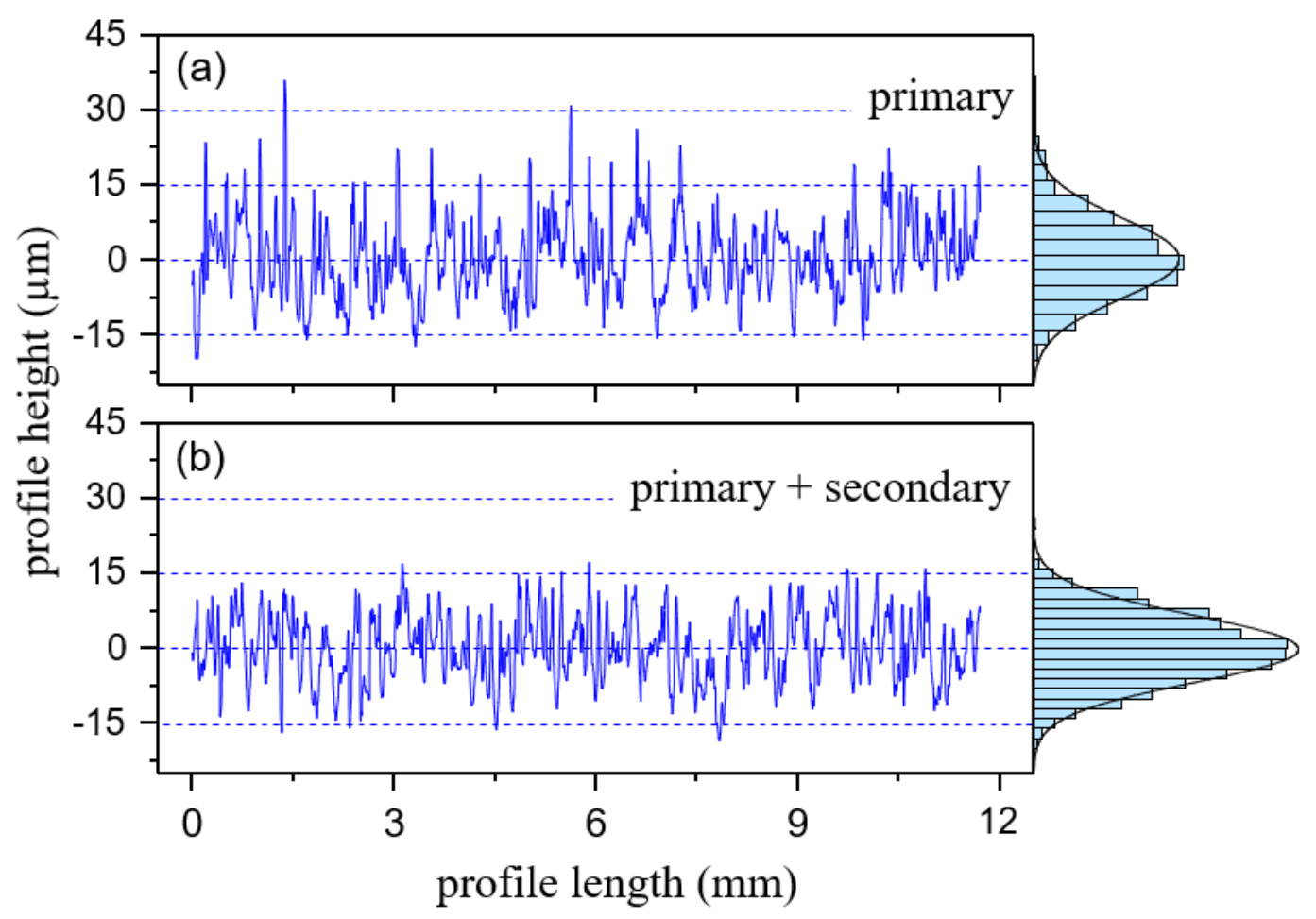

Fig 4.3.7 Profiles and histograms of the surfaces generated by (a) primary texturing only and (b) primary + secondary texturing with the distribution histogram on the side.

During the machining process, the spots with the shortest distance between the electrode and workpiece would generate sparks. Therefore, valleys would not experience any machining before the peaks were removed. On the other hand, the finishing parameters were 1.2 A for the current and $0.4 \mu$ s for the discharge duration, which were the smallest that the machine could generate. Superhydrophobic hierarchical structures were usually combined with micro and nano level features; however, the secondary machining of EDM 
could not generate craters in nano levels. This conclusion also confirmed that to generate hydrophobic surfaces by using Sink EDM was not to fabricate hierarchical structures, but to rather create surfaces similar to pillar arrayed surfaces.

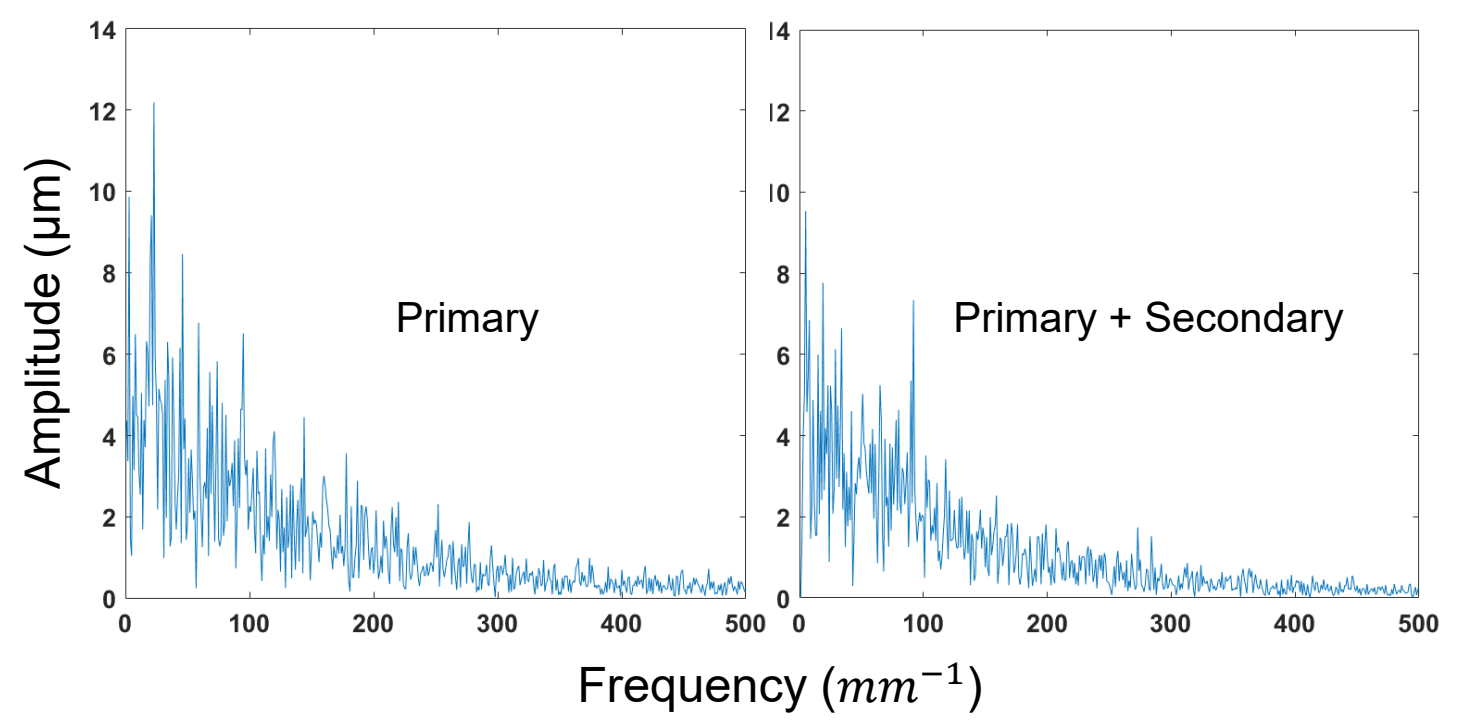

Fig. 4.3.8 Fast Fourier Transform of the surface profiles shown in Fig. 4.3.7.

\subsubsection{Workpiece Material Al 7075 Vs. Al 6061}

Aluminum 7075 alloy is one of the most commonly used aluminum alloys in marine and aerospace industries. Its higher strength-to-weight ratio and corrosion resistance provide Al 7075 a totally different applicable area than other grades of aluminum alloys. Its low surface free energy makes it suitable in fabricating hydrophobic surfaces. However, due to the limitation of its machinability, $\mathrm{Al} 6061$ is more widely used for general purposes, like automotive industries and food packaging. A list of the chemical compositions for both two alloys is illustrated in Table 4.2. 
Table 4.2 Chemical Composition of Aluminum Alloy $6061 \& 7075$

\begin{tabular}{|c|ccccc|}
\hline$(\%)$ & $\mathrm{Si}$ & $\mathrm{Fe}$ & $\mathrm{Cu}$ & $\mathrm{Mn}$ & $\mathrm{Mg}$ \\
\hline $\mathrm{Al} 6061$ & $0.4-0.8$ & 0.7 & $0.15-0.4$ & 0.15 & $0.8-1.2$ \\
$\mathrm{Al} 7075$ & 0.4 & 0.5 & $1.2-2$ & 0.3 & $2.1-2.9$ \\
\hline$(\%)$ & $\mathrm{Cr}$ & $\mathrm{Zn}$ & $\mathrm{Ti}$ & Other & $\mathrm{Al}$ \\
\hline $\mathrm{Al} 6061$ & $0.04-0.35$ & 0.25 & 0.15 & 0.15 & $\mathrm{Rem}$. \\
$\mathrm{Al} 7075$ & $0.18-0.28$ & $5.1-6.1$ & 0.2 & 0.15 & Rem. \\
\hline
\end{tabular}

Compared with Al 6061, Al 7075 contains a bigger amount of 'non-aluminum' metals especially Zinc. Zinc alloyed aluminum is more heat-treatable and hard comparing to other alloys. These two grades of aluminum alloys are different in mechanical properties, but similar in thermal properties. As a melting process, EDM machining is able to construct similar structures on both materials. An experiment was designed to investigate the difference in contact angle between these two alloys. Meantime, applicable surfaces were also desired for both materials; therefore, the parameters that could generate surfaces with high hydrophobicity were used for both alloys. The machining parameters used a current of $29 \mathrm{~A}$ and discharge duration of $18 \mu \mathrm{s}$ for the primary texturing. Varied secondary machining durations were used on the top of the primary texturing, ranged from 5 to 35 seconds. Results are shown in Fig. 4.3.9, all the contact angles, in both groups, were around $140^{\circ}$ to $142^{\circ}$, which indicated no significant difference. Firstly, this result meant that the final textured surfaces with both alloys contained similar structure, roughness, as well as 
surface chemical compositions. Secondly, Sink EDM machining of high water-repellent surfaces could be accomplished by using both Al 6061 and Al 7075.

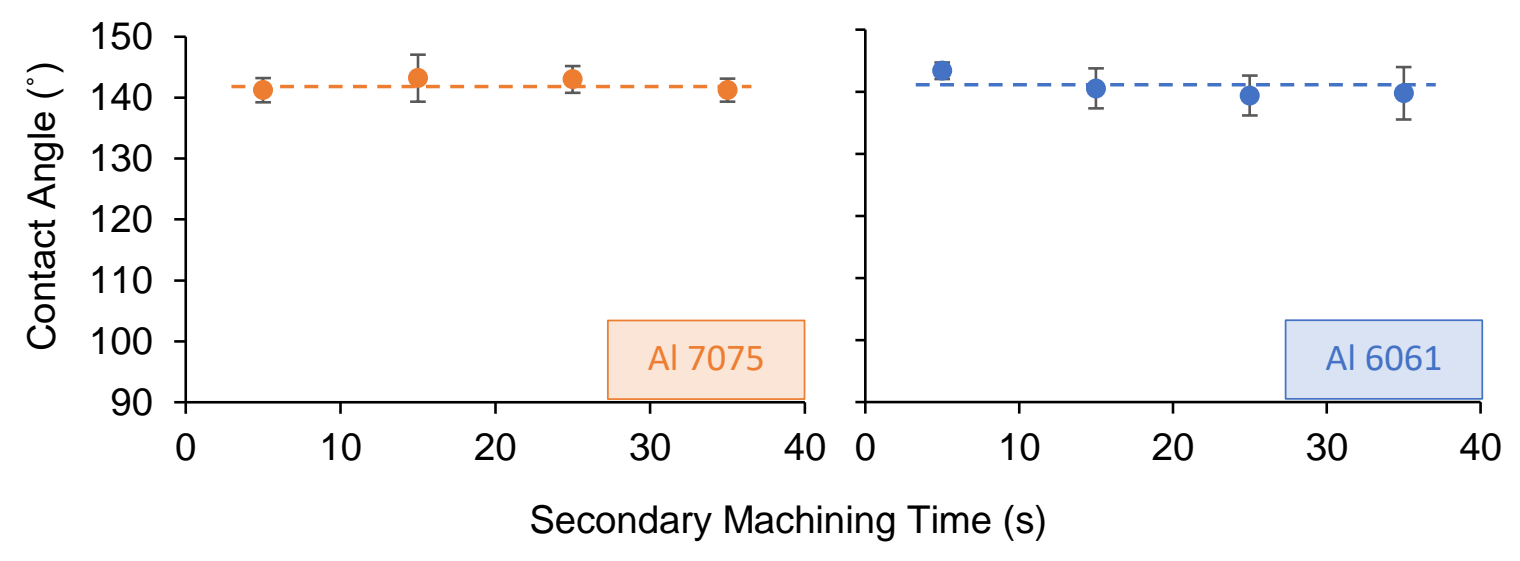

Fig 4.3.9 Contact angle Vs. Secondary machining time for Al 7075 and Al 6061 alloys.

\subsubsection{Contact Angle Degradation by Using EDMed Electrode Surfaces}

As mentioned before, an electrode with a milled surface was used for every new experiment for consistency. An electrode surface with 'EDM texturing' was never used for the next machining, which was not suitable for industrial applications. The electrode degradation related to the number of EDM machining cycles was tested and studied. Three milled electrodes were prepared, and each of them did five cycles of the primary texturing process. Contact angles were measured on all the workpiece surfaces, and the average value was calculated by using the surfaces machined in the same number of orders. Mean values and the standard deviations are shown in Fig. 4.3.10. 


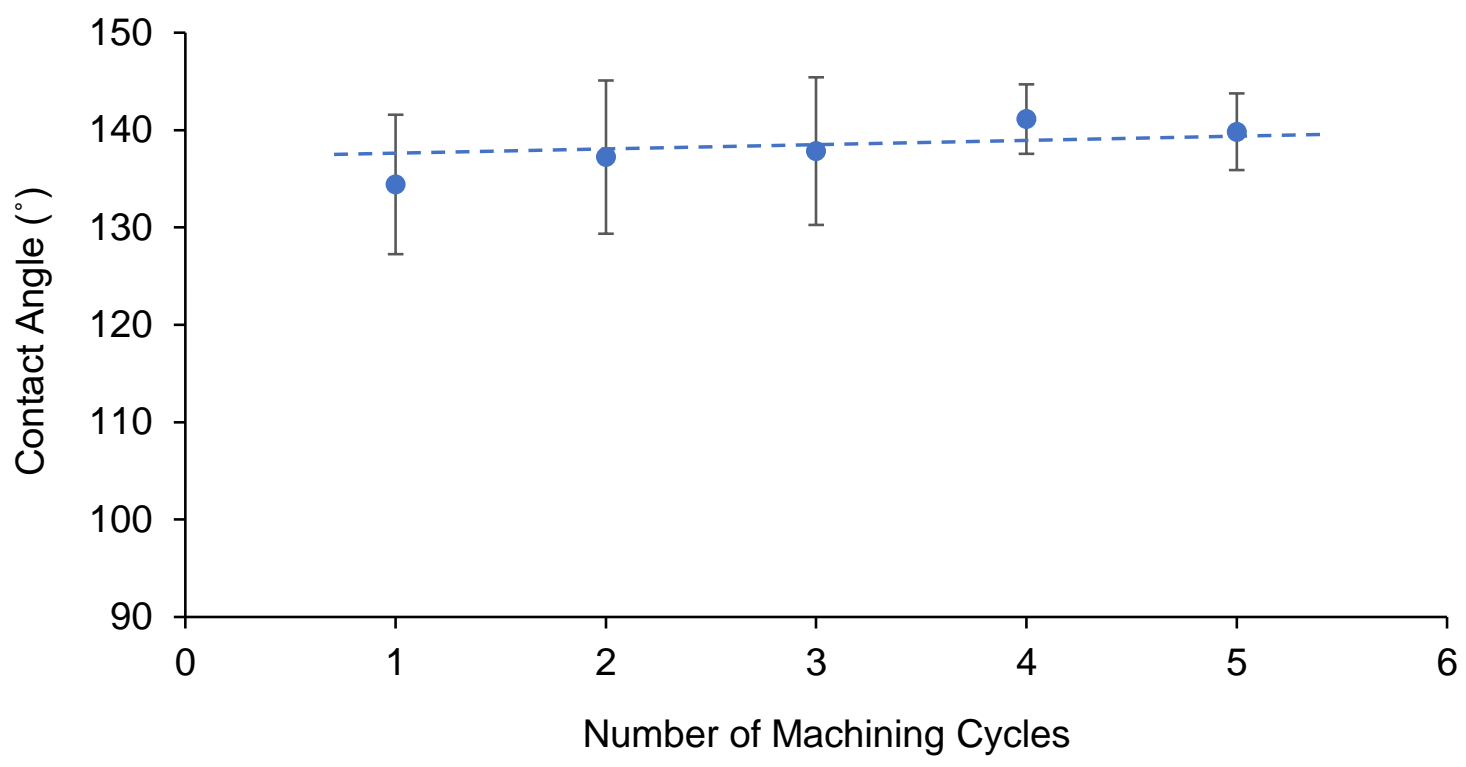

Fig. 4.3.10 Contact angle Vs. Number of machining times of used electrode surfaces.

According to the five data points, no obvious difference is observed, which means the same electrode surface can machine more than one workpiece without changing the wetting behaviour of the textured surfaces. This can save a great amount of energy and time in industrial applications. In this experiment, each electrode only machined five workpieces for the study. Higher numbers can be tested for the future work. In addition, same machining parameters were used for all the surfaces in this experiment. Varying the spark energy would definitely change the surface structure of the electrodes, which would also affect the surface structure of the workpiece. In terms of wettability, the changes would be complicated to analyze in this case due to the large number parameters. Therefore, same machining conditions should be used if the electrode was desired to be used for more than one cycle of machining at present. 


\subsubsection{Sink EDM Experiment in Water and Time Influence}

As the most interesting section of the study, an extreme condition was obtained through the water experiment. Hydrophilic or even superhydrophilic surfaces were obtained by using water as the dielectric medium. To simplify the experiment, surfaces machined by primary texturing only was conducted in both dielectric oil and water. The machining parameters were $29 \mathrm{~A}$ and $18 \mu \mathrm{s}$ for the current and discharge duration respectively. A contact angle of $18^{\circ}$ was measured on the surface machined in water, and $135^{\circ}$ in oil. Same as many laser beam machined surfaces introduced in chapter 3, the surfaces became hydrophobic or superhydrophobic after a certain period. In this study, this period was named 'curing time'. Sample pieces were machined with the same parameters but different dielectric mediums. Contact angles were followed for a month to investigate influence of curing time. Results are shown in Fig. 4.3.11.

Contact angles of curve (a) were measured on the surfaces machined in the hydrocarbon oil, and then placed in a box which was exposed to air. An increase of approximately 10 degrees was observed after 30 days of curing time comparing to the first day. This was because aluminum surfaces could absorb carbon particles from air to reduce the surface free energy [46]. Surfaces machined in hydrocarbon oil were hydrophobic immediately after machining, because the dielectric medium contained carbon element which could deposit onto workpiece surfaces. This explained why the surfaces machined

in deionized (DI) water (b) indicated hydrophilicity at the beginning but became hydrophobic after 30 days which indicated an increase of $125^{\circ}$ in contact angle. Another test (c) was constructed to verify that the increase in hydrophobicity for the surfaces should 
be accomplished in air, or somewhere that contained carbon element. The surfaces left in DI water after machining became superhydrophilic, and the completely-wetting behaviour remained if the sample specimens were kept in DI water. A white layer was formed on the surfaces, and it might be a layer of the metallic oxides, which were hydrophilic materials. The analysis of this white layer may need some future work for a further investigation.

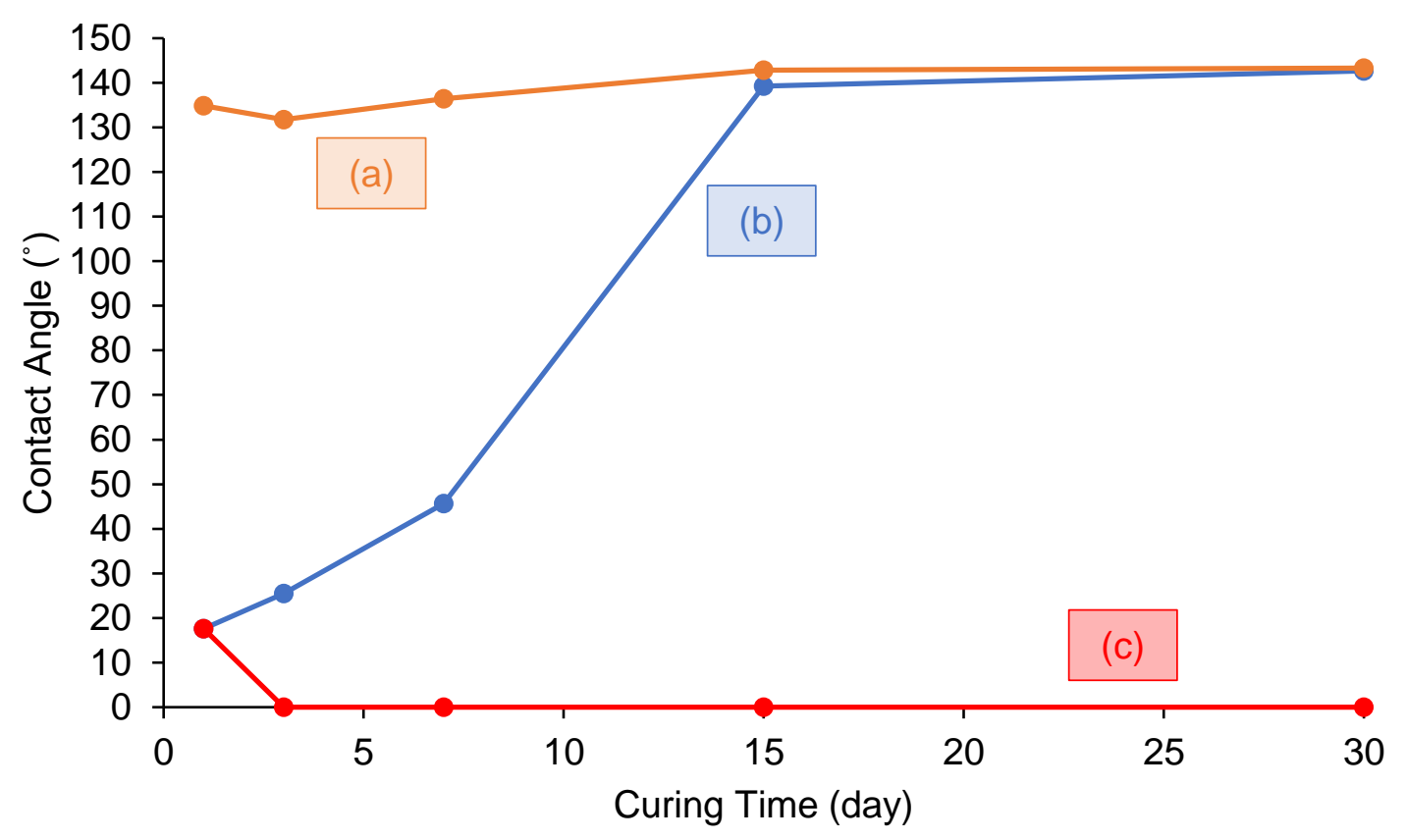

Fig. 4.3.11 Contact angle Vs. Curing time for three groups of machining: (a) machined in hydrocarbon oil, (b) machined in DI water, and (c) machined in DI water, then keep the workpieces in DI water.

Before conducting the surface chemistry analysis, surface profiles of the workpieces machined in both DI water and hydrocarbon oil were examined first, shown in Fig 4.3.12. No significant difference could be observed, which meant the big variability of contact angles between surfaces machined in water and oil was not caused by the structure change. 
The profile extraction was followed by an FFT analysis (Fig 4.3.13), and similar results were obtained.

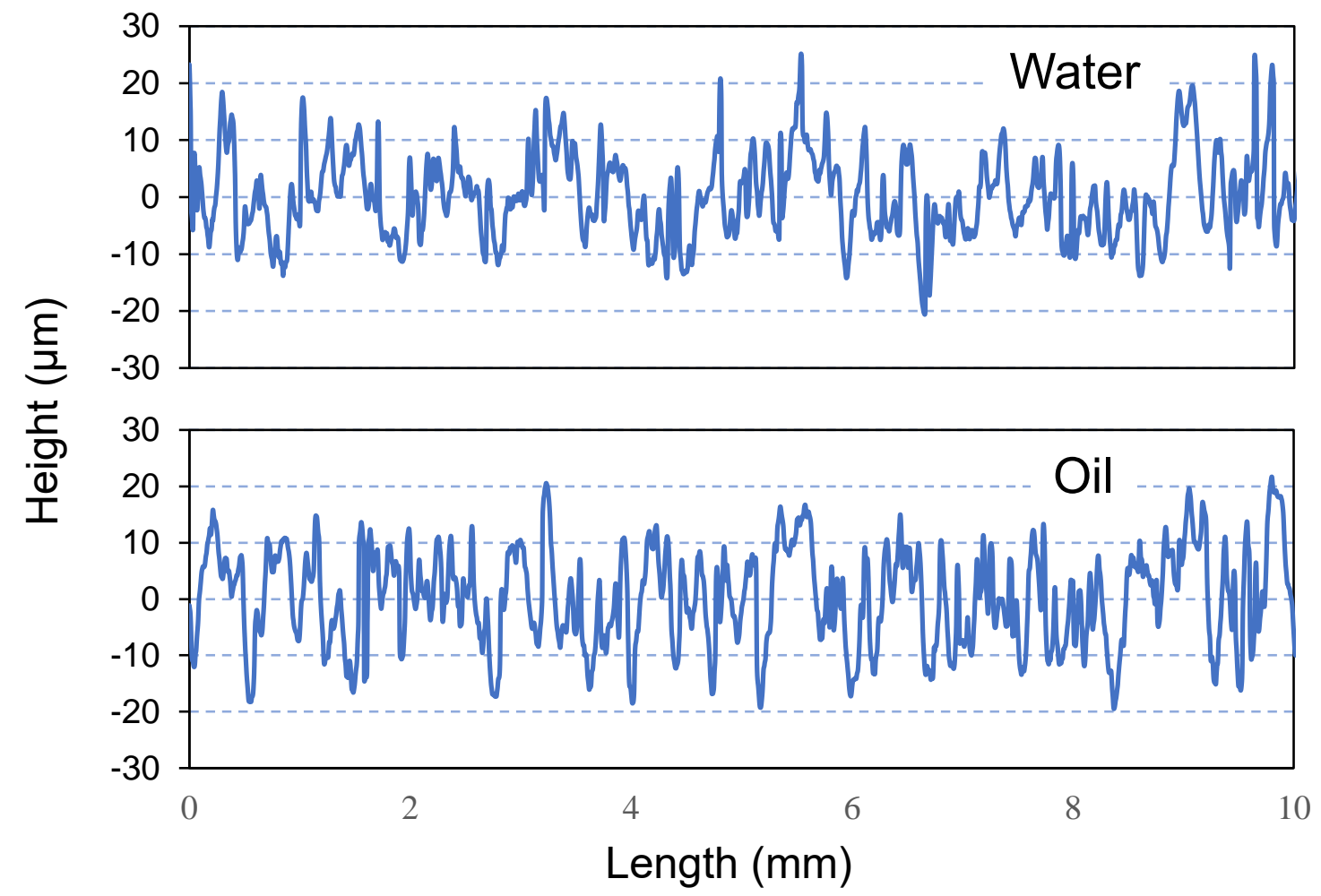

Fig. 4.3.12 Profiles of the surfaces machined in water and oil.

SEM and EDS analysis were also performed to confirm the conclusions made for (a) and (b) in Fig. 4.3.11. Surface chemistry of three workpieces was examined, and the results are shown in Fig. 4.3.14. Comparing (1) and (2), the level of carbon was similar which meant that lack of carbon was not the reason why surfaces machined in water were hydrophilic. The most obvious difference was the amount of oxygen element on the surfaces. There was only a small amount of oxygen found from the surface machined in oil but $27 \%$ on the surface machined in water. The oxygen element from surface (1) was most 
likely in the form of aluminum oxides and a small amount of other oxides which had hydrophilic properties. Surfaces machined in hydrocarbon oil did not have any oxygen during the machining process which resulted in very few amount of $\mathrm{O}$ element.

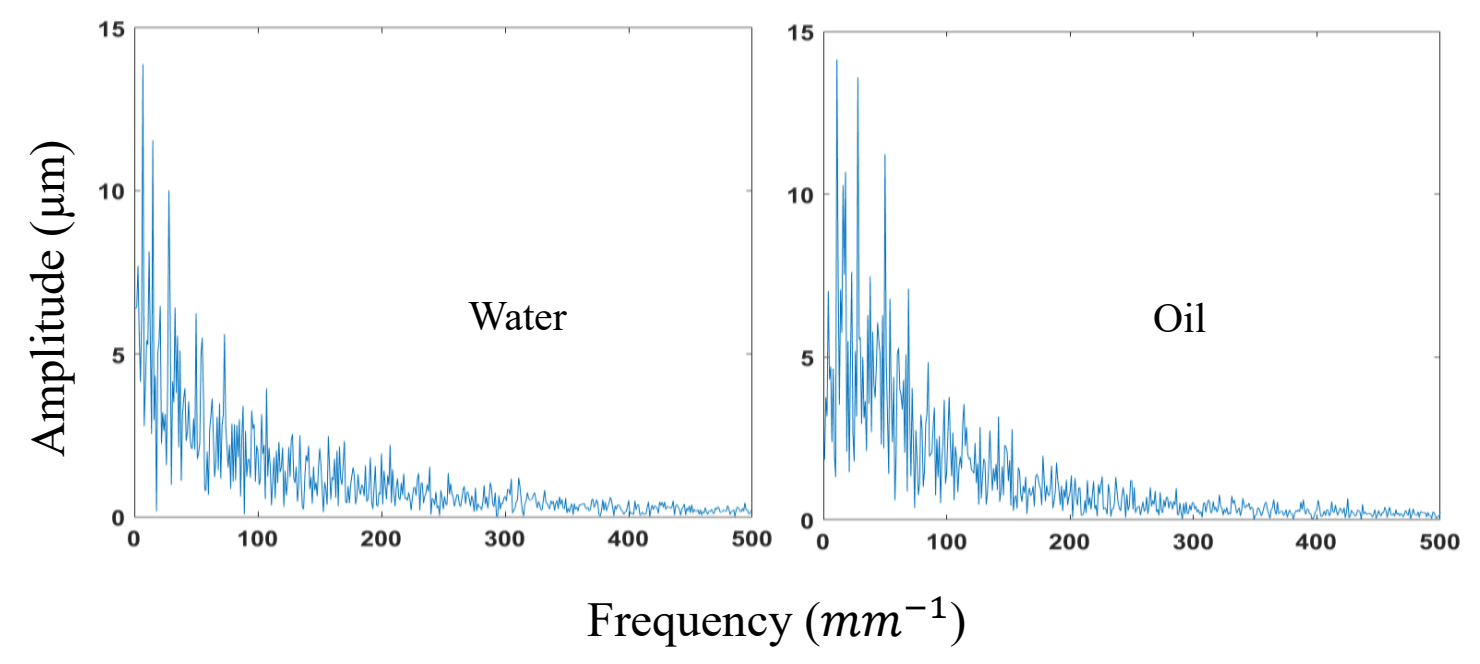

Fig. 4.3.13 Fast Fourier Transform of the surfaces shown in Fig. 4.3.12.

On the other hand, the surface exposed to air after 15 days machined in oil indicated an increase in carbon level from $10.9 \%$ to $19.1 \%$, which matched the conclusion made before. Oxygen level also increased from $3.1 \%$ to $8.1 \%$, which meant the oxide layer could also be from on this surface. An increase in the contact angle explained that the effect of carbon deposition played a more important role than the oxide layer. The increase of contact angle would reach a saturation point after approximately 15 to 20 days, which would due to the slower increasing rate of carbon level. The superhydrophobic surfaces introduced in section 4.1 was machined in hydrocarbon oil and left in air for half a month. Immediately after machining, a contact angle around $143.2^{\circ} \pm 2.20^{\circ}$ was observed which already 
indicated hydrophobicity. This would correspond to a contact angle of $155.4^{\circ}$ could be obtained if Y-L fitting method was used.

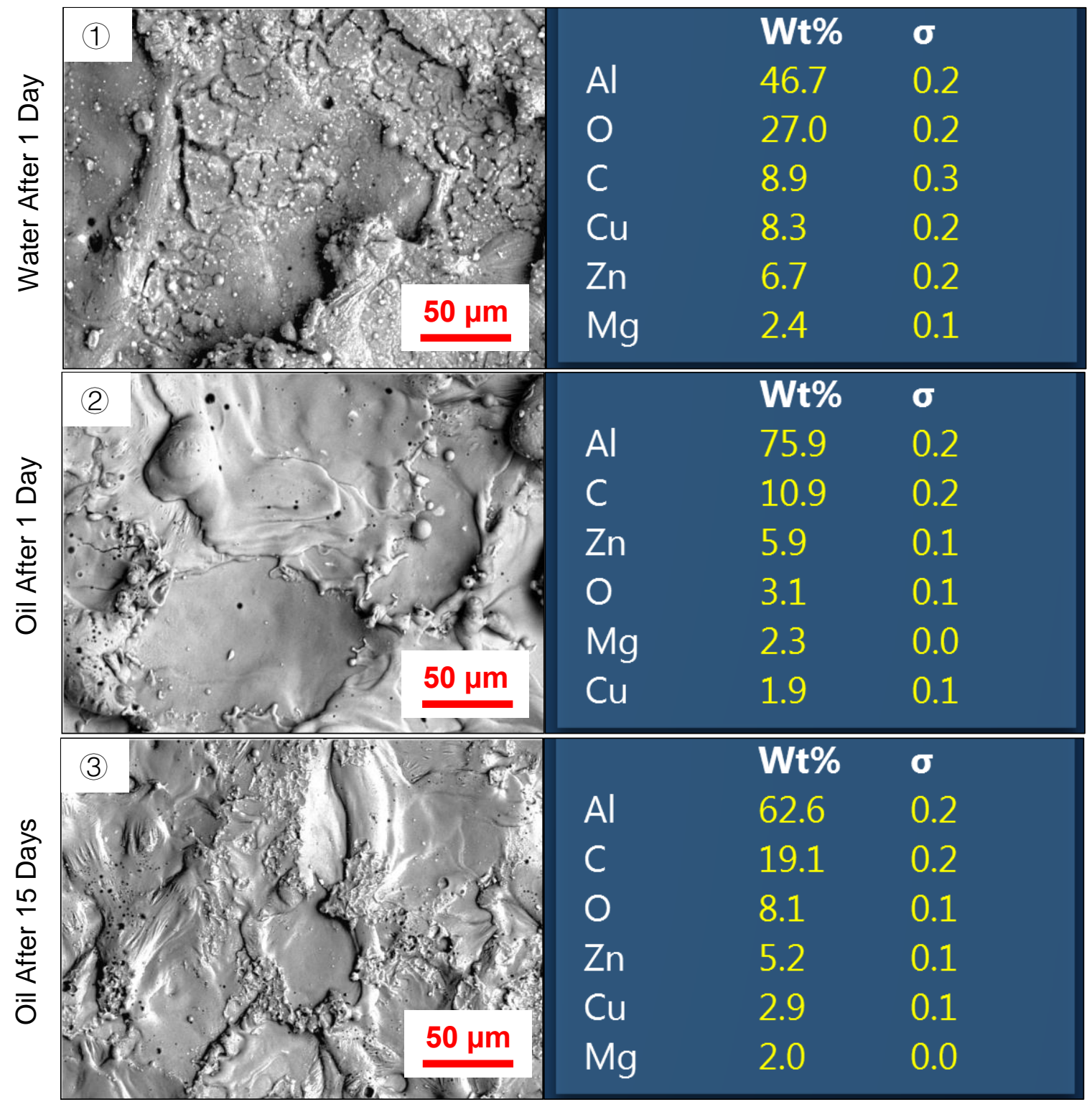

Fig 4.3.14 SEM and EDM analysis for three surfaces: (1) one day after machined in DI water, (2) one day after machined in hydrocarbon oil, and (3) 15 days after machined in hydrocarbon oil. 


\subsection{PDMS Casting}

PDMS is well known as one of the most hydrophobic materials with an intrinsic contact angle of $111^{\circ} \pm 2^{\circ}$. A PDMS casting experiment was constructed to test if the opposite structure of the textured surfaces could also perform a non-wetting property. In addition, if the casted surfaces indicated a superhydrophobic behaviour, then a one-step molding technique could be reported. Surfaces with three different roughness levels were tested in this experiment, and the results are shown below in Table 4.3.

Table 4.3 Contact Angles on Casted PDMS and Relative Aluminum Alloy Surfaces

\begin{tabular}{|c|c|c|c|}
\hline Machining Parameters & $6.2 \mathrm{~A} 3.2 \mu \mathrm{s}$ & $17 \mathrm{~A} 18 \mu \mathrm{s}$ & $52 \mathrm{~A} 56 \mu \mathrm{s}$ \\
\hline Aluminum Surface & $131.0^{\circ}$ & $135.5^{\circ}$ & $128.0^{\circ}$ \\
\hline Casted PDMS & $133.4^{\circ}$ & $130.3^{\circ}$ & $120.9^{\circ}$ \\
\hline
\end{tabular}

The contact angles did not increase on the casted PDMS surfaces, rougher surfaces even experienced a decreasing trend. According to the data shown above, two more surfaces were tested. The first surface was a finer textured surface which was used to investigate if the contact angle could further increase the contact angle. The machining parameters for such surface were 6.2 A for the current and $2.1 \mu$ s for the discharge duration, and the casted PDMS surface had a contact angle of $130.6^{\circ}$. This result meant that to further decrease the roughness of the surfaces would not be able to help enhance the 
hydrophobicity. The second test used a dual-structural surface which was machined on the top of the roughest sample piece. A secondary texturing of 20 seconds was performed, but a contact angle of $114.1^{\circ}$ was obtained on the casted surface. The value was only $3^{\circ}$ higher than what could be observed on a flat PDMS surface. Therefore, the dual structure also could not help to improve the hydrophobicity.

In conclusion, the opposite structure of the EDMed surfaces, which was fabricated by one-step PDMS casting process, could not generate superhydrophobic surfaces. The increase in contact angle comparing to a flat PDMS surface was due to the roughness, which could prevent the water from spreading. One more step might be needed to duplicate the EDM textured structures to fabricate surfaces with higher non-wettability. 


\section{Chapter 5}

\section{Conclusions and Future Work}

\subsection{Conclusions}

This work was motivated by the challenges in fabricating a robust superhydrophobic surface on metals. It was proposed that EDM process could fabricate such surfaces at a fast machining rate. Several studies into the factors influencing surface hydrophobicity were investigated, and the results are summarized and listed below:

1. An intrinsic contact angle of $59.4^{\circ}$ was measured on a polished Aluminum 7075 alloy surface. After EDM texturing, with parameters of 29 A and $18 \mu$ s for the primary texturing and $1.2 \mathrm{~A}$ and $0.4 \mu \mathrm{s}$ for the secondary texturing, a contact angle of $143.2^{\circ} \pm 2.2^{\circ}$ was obtained after one day of machining. The value increased to $149.4^{\circ} \pm 0.77^{\circ}$ with a contact angle hysteresis of $15.3^{\circ} \pm 2.62^{\circ}$ after 15 days when the surfaces were exposed to air. Impacting droplets fully bounced off the surfaces which further indicated non-wetting behaviour.

2. Surfaces were machined with different discharge energies to study the influence of surface topography and roughness on contact angle. Small current and discharge duration (e.g. 6.2 A $6.5 \mu \mathrm{s}$ ) could generate finer surfaces and the contact angles were generally bigger. Smaller contact angles were found on the surfaces machined by using larger parameters due to larger craters. Water drops could penetrate the surfaces more easily when 
peaks were sparse. Smaller contact angles were also observed on the surfaces with ultrasmall machining parameters because the tiny structures could not hold the water drop, and such surfaces acted more similarly to polished surfaces.

3. A secondary texturing was conducted on the top of primary textured surfaces which could remove the sharp peaks on the surfaces so that the surfaces were more uniform and homogeneous. This could reduce the variability of contact angle measurement for finer surfaces, but the contact angles remained the same because the finishing process could not make significant change when surfaces were fine enough. On the other hand, for rougher surfaces, the finishing process could not reduce the variability significantly, but the contact angles were increased after removing the peaks. To enhance the hydrophobicity, longer secondary texturing times were needed for surfaces with higher roughness. Excessive secondary machining would decrease the contact angle at a slow rate.

4. Aluminum 6061 alloy was machined by using the same parameters as aluminum 7075 alloy. With primary texturing parameters of 29 A for the current, $18 \mu$ s for the discharge duration, a contact angle around $140^{\circ}$ could be obtained after the secondary texturing. The results were very similar to aluminum 7075 alloy, which indicated that EDM could fabricate surfaces with high hydrophobicity on both these materials.

5. The same copper electrode surface was used to machine more than one sample, and no significant difference in contact angle was observed on workpiece surfaces. This could potentially save a great amount of time and energy in the electrode preparation process. 
6. Several workpieces were machined by using deionized water as the dielectric medium, and hydrophilicity was first observed on their surfaces with a contact angle of around $18^{\circ}$. Same parameters were used to machine some workpieces in dielectric oil, and hydrophobic surfaces were obtained with a contact angle around $135^{\circ}$. Surface characterization indicated that the difference in contact angle originated from the surface chemistry but not the surface structure. Samples machined in DI water revealed a higher level of elemental oxygen, which might be metallic oxides that are hydrophilic. After samples exposing to air for 30 days, contact angles of all the surfaces exceeded $140^{\circ}$ due to the increase in elemental carbon level.

7. A PDMS casting process was conducted to investigate the wettability of the complementary structure of EDM texturing. Surfaces with different roughness levels were used for this study, and finer surfaces indicated bigger contact angles on cast PDMS surfaces. However, as one of the most hydrophobic materials with an intrinsic contact angle of $111^{\circ}$, the highest contact angle obtained was $133^{\circ}$, which meant that the replicated surfaces indicated lower wettability compared to EDM textured surfaces.

This work demonstrated a novel technology for fabricating metallic hydrophobic surfaces by using EDM. Contact angle was measured in terms of machining parameters, dielectric medium, and workpieces materials, which points to its application in industry. 


\subsection{Future Work}

In this section, future work is suggested to better understand the hydrophobicity of EDM textured surfaces.

1. Surfaces machined by using DI water indicated hydrophilicity immediately after machining but became hydrophobic after approximately 10 days when they were exposed to air. An EDS analysis was conducted; however, no significant change was observed as shown in Fig. 5.2.1. Elemental carbon played an important role in wettability, but EDS analysis was not accurate enough for carbon level analysis. A further investigation with more advanced equipment like Wavelength Dispersive X-ray Spectroscopy (WDS) can be helpful.

\begin{tabular}{|lll|lll|} 
& $\boldsymbol{W t} \%$ & $\boldsymbol{\sigma}$ & & $\boldsymbol{W t} \%$ & $\boldsymbol{\sigma}$ \\
$\mathrm{Al}$ & 46.7 & 0.2 & $\mathrm{Al}$ & 45.5 & 0.2 \\
$\mathrm{O}$ & 27.0 & 0.2 & $\mathrm{O}$ & 26.1 & 0.2 \\
$\mathrm{C}$ & 8.9 & 0.3 & $\mathrm{C}$ & 9.9 & 0.3 \\
$\mathrm{Cu}$ & 8.3 & 0.2 & $\mathrm{Cu}$ & 9.6 & 0.2 \\
$\mathrm{Zn}$ & 6.7 & 0.2 & $\mathrm{Zn}$ & 6.3 & 0.1 \\
$\mathrm{Mg}$ & 2.4 & 0.1 & $\mathrm{Mg}$ & 2.5 & 0.0 \\
\hline \multicolumn{3}{c}{ After 1 day } & \multicolumn{3}{c}{ After 15 days }
\end{tabular}

Fig. 5.2.1 Surface chemistry analysis by using EDS for the surfaces machined after (left) 1 day and (right) 15 days. 
2. Two types of workpiece materials were studied in this thesis: Aluminum 7075 and 6061 alloys. Other than Al alloys, commonly used materials like stainless steel, titanium alloy could be tested to investigate if high contact angles can be obtained.

3. Al 7075 can be used as tool electrode and workpiece material simultaneously which may enhance the fabrication efficiency by $100 \%$. The surface topography is possibly different as compared to using copper as the electrode, even the parameters are the same. Some effort is needed to investigate the suitable current and discharge duration that can help maximize the contact angle.

4. Al 7075 or Al 6061 are essentially hydrophilic materials; therefore, some surface modification techniques may be helpful to improve hydrophobicity by reducing the surface free energy. Surface coating with some low energy polymers like parylene, PDMS, or Teflon can be suitable options.

5. Referring to Fig. 4.3.14, some small features could be observed on different surfaces in SEM images. When using hydrocarbon oil as the dielectric medium, after 15 days of machining the surface appears not as smooth as the surface machined after one day. This is possibly a reason why contact angle increases, because these small features may refer to a nano texturing process, which forms a hierarchical structure. Further investigation is needed to prove if this hypothesis is true. 


\section{Chapter 6}

\section{References}

[1] T. Young, "An essay on the cohesion of fluids". Philosophical Transactions of the Royal Society of London, Vol. 95 page 65-87, 1805

[2] K.-Y. Law, and H. Zhao, "Contact angle measurements and surface characterization techniques”. Surface Wetting, page 7-9, 2016

[3] L. Rayleigh, "On the tension of water surfaces, clean and contaminated, investigated by the method of ripples". The London, Edinburgh, and Dublin Philosophical Magazine and Journal of Science, Vol. 30 page 386-400, 1890

[4] F. E. Bartell, and Allan D. Wooley, "Solid-liquid-air contact angles and their dependence upon the surface condition of the solid". Journal of the American Chemical Society, Vol. 55 (9) page 3518-3527, 1933

[5] R. N. Wenzel, "Resistance of solid surfaces to wetting by water". Industrial \& Engineering Chemistry, Vol. 28 (8) page 988-994, 1936

[6] A. B. D. Cassie, and S. Baxter, "Wettability of porous surfaces". Transactions of the Faraday Society, Vol. 40 page 546-551, 1944

[7] R. E. JohnsonJr, and R. H. Dettre, "Contact angle hysteresis. III. Study of an idealized heterogeneous surface". The Journal of Physical Chemistry, Vol. 68 (7) page 17441750,1964 
[8] B. Wilhelm, and E. Nesta, "Scanning electron microscopy of the epidermal surfaces of spermatophytes". Tropical and Subtropical Plant World, Vol. 19 page 69-110, 1977

[9] S. Wang, and L. Jiang, "Definition of Superhydrophobic States". Advanced Materials, Vol. 19 (21) page 3423-3424, 2007

[10] Z. Ahmad, S. Rehman, "International review of Mechanical Engineering”. Vol. 3 (3) 312, 2009

[11] C. E. Cansoy, "The effect of drop size on contact angle measurements of superhydrophobic surfaces”. Royal Society of Chemistry, Vol. 4 (4) page 1197-1203, 2014

[12] M. Kunieda, B. Lauwers, K. P. Rajurkar, and B. M. Schumacher, "Advancing EDM through fundamental insight into the process". Cirp Annals-Manufacturing Technology, Vol. 54 (2) page 64-87, 2005

[13] R. S. Prakash, M. Sivakumar, M. Jeevaraja, and G. Saravanan, "Review on wire electrical discharge machining of die and tool grade steels". International Journal of Applied Engineering Research, Vol. 10 (85) page 521-527, 2015

[14] S. H. Kim, "Fabrication of superhydrophobic surfaces". Journal of Adhesion Science and Technology, Vol. 22 (3-4) page 235-250, 2012

[15] M. Ma, and R. M. Hill, "Superhydrophobic surfaces". Current Opinion in Colloid \& Interface Science, Vol 11 (4) page 193-202, 2006 
[16] Y. Y. Yan, N. Gao, and W. Barthlott, "Mimicking natural superhydrophobic surfaces and grasping the wetting process: A review on recent progress in preparing superhydrophobic surfaces”. Advances in Collioid and Interface Science, Vol. 169 (2) page $80-105,2011$

[17] V. Kovacevic, "Surface and interface phenomenon in polymers". International Workshop on Advanced Polymer Science and Turbulent Drag Reduction, Vol. 1984 (3), 2008

[18] A. M. Kietzig, S. G. Hatzikiriakos, and P. Englezos, "Patterned superhydrophobic metallic surfaces”. Langmuir Article, Vol. 25 (8) page 4821-4827, 2008

[19] V. D. Ta, A. Dunn, T. J. Wasley, J. Li, R. W. Kay, J. Stringer, P. J. Smith, E. Esenturk, C. Connaughton, and J. D. Shephard, "Laser textured superhydrophobic surfaces and their applications for homogeneous spot deposition". Applied Surface Science, Vol. 365 (1) page 153-159, 2016

[20] D. M. Chun, Chi-Vinh Ngo, and Kyong-Min Lee, "Fast fabrication of superhydrophobic metallic surface using nanosecond laser texturing and low-temperature annealing”. Cirp Annals - Manufacturing Technology, Vol. 65 (1) page 519-522, 2016

[21] C. V. Ngo, and D. M. Chun, "Fabrication of un-coated transparent superhydrophobic sapphire surface using laser ablation and heat treatment". Cirp Annals - Manufacturing Technology, Vol. 67 (1) page 571-574, 2018

[22] B. Li, H. Li, L. Huang, N. Ren, and X, Kong, "Femtosecond pulsed laser textured titanium surfaces with stable superhydrophilicity and superhydrophobicity". Applied Surface Science, Vol. 389 (15) page 585-593, 2016 
[23] B. Farshchian, J. R. Gatabi, S. M. Bernick, S. Park, Gwan-Hyoung Lee, R. Droopad, and N. Kim, "Laser-induced superhydrophobic grid patterns on PDMS for droplet arrays formation”. Applied Surface Science, Vol. 396 (28) page 359-365, 2017

[24] C. V. Ngo, and Doo-Man Chun, "Fast wettability transition from hydrophilic to superhydrophobic laser-textured stainless steel surfaces under low-temperature annealing”. Applied Surface Science, Vol. 409 (1) page 232-240, 2017

[25] B. Wu, M. Zhou, J. Li, X. Ye, G. Li, and L. Cai, "Superhydrophobic surfaces fabricated by microstructuring of stainless steel using a femtosecond laser”. Applied Surface Science, Vol. 256 (1) page 61-66, 2009

[26] R. Jagdheesh, B. Pathiraj, E. Karatay, G. R. B. E. Romer, and A.J. Huis in’t Veld, “Laserinduced nanoscale superhydrophobic structures on metal surfaces”. Langmuir Article, Vol. 27 (13) page 8464-8469, 2011

[27] W. G. Bae, K. Y. Song, Y. Rahmawan, C. N. Chu, D. Kim, D. K. Chung, and K. Y Suh, “One step process for superhydrophobic surfaces by wire electrical discharge machining”. ACS Applied Materials \& Interfaces, Vol. 4 (17) page 3685-3691, 2012

[28] J. Y. So, and W. G. Bae, "Fabrication of superhydrophobic metallic surface by wire electrical discharge machining for seamless roll-to-roll printing”. Metals, Vol. 8 (4), 2018

[29] W. G. Bae, D. Kim, K. Y. Song, H. E. Jeong, and C. N. Chu, "Engineering stainless steel surface via wire electrical discharge machining for controlling the wettability". Surface \& Coatings Technology, Vol. 275 (15) page 316-323, 2015 
[30] P. B. Weisensee, E. J. Torrealba, M. Raleigh, A. M. Jacobi, and W. P. King, "Hydrophobic and oleophobic re-entrant steel microstructures fabricated using micro electrical discharge machining”. Journal of Micromechanics and Microengineering, Vol. 24 (9) page 095020, 2014

[31] S. Dong, Z. Wang, Y. Wang, X. Bai, Y. Qing, R. Fu, B. Guo, C. Tan, J. Zhang, and P. $\mathrm{Hu}$, "Roll-to-roll manufacturing of robust superhydrophobic coating on metallic engineering materials". ACS Applied Materials \& Interfaces, Vol. 10 (2) page 2174-2184, 2018

[32] Y. Zhang, X. Yu, Q. Zhou, F. Chen, and K. Li, "Fabrication of superhydrophobic copper surface with ultra-low water roll angle”. Applied Surface Science, Vol. 256 (6) page $1883-1887,2010$

[33] Y. Shen, H. Tao, S. Chen, Y. Xie, T. Zhou, T. Wang, and J. Tao, "Water repellency of hierarchical superhydrophobic Ti6Al4V surfaces improved by secondary nanostructures”. Applied Surface Science, Vol. 321 (1) page 469-474, 2014

[34] J. J. Victor, D. Facchini, and U. Erb, “A low-cost method to produce superhydrophobic polymer surfaces”. Journal of Materials Science, Vol. 47 (8) page 3690-3697, 2012

[35] X. Fu, and X. He, "Fabrication of super-hydrophobic surfaces on aluminum alloy substrates”. Applied Surface Science, Vol. 255 (5-1) page 1776-1781, 2008

[36] H. -D. Yu, X. -R. Zhang, Y. -L. Wan, J. -K. Xu, Z. -J. Yu, and Y. -Q. Li, “Superhydrophobic surface prepared by micromilling and grinding on aluminum alloy". Surface Engineering, Vol. 32 (2) page 107-113, 2016 
[37] Z. Shi, Z. Liu, H. Song, and X. Zhang, "Prediction of contact angle for hydrophobic surface fabricated with micro-machining based on minimum Gibbs free energy". Applied Surface Science, Vol. 364 (28) page 597-603, 2016

[38] P. Guo, Y. Lu, K. F. Ehmann, and J. Cao, "Generation of hierarchical micro-structures for anisotropic wetting by elliptical vibration cutting”. Cirp Annals - Manufacturing Technology, Vol. 63 (1) page 553-556, 2014

[39] M. Kunieda, and T. Kobayashi, "Clarifying mechanism of determining tool electrode wear ratio in EDM using spectroscopic measurement of vapor density". Journal of materials processing technology, Vol. 149 (1-3) page 284-288, 2004

[40] X. Zhang, F. Shi, J. Niu, Y. Jiang, and Z. Wang, "Superhydrophobic surfaces: from structural control to functional application”. Journal of Materials Chemistry, Vol. 18 (6) page $621-633,2008$

[41] M. Hadad, L. Q. Bui, and C. T. Nguyen, "Experimental investigation of the effects of tool initial surface roughness on the electrical discharge machining (EDM) performance". The international Journal of Advanced Manufacturing Technology, Vol. 95 (5-8) page 2093-2104, 2018

[42] M. Callies, Y. Chen, F. Marty, A. Pepin, and D. Quere, "Micorfabricated textured surfaces for super-hydrophobicity investigations". Microelectronic Engineering, Vol. 78-79 page $100-105,2005$

[43] K. K. Varanasi, T. Deng, M. Hsu, and N. Bhate, "Hierarchical superhydrophobic surfaces resist water droplet impact". Technical Proceedings of the 2009 NSTI Nanotechnology Conference and Expo, 2009 
[44] J. Yeo, M. J. Choi, and D. S. Kim, "Robust hydrophobic surfaces with various micropillar arrays". Journal of Micromechanics and Microengineering, Vol. 20 (2) 025028,2010

[45] Q. Zheng, and C. Lv, "Size effects of surface roughness to superhydrophobicity". Procedia IUTAM, Vol. 10 page 462-475, 2014

[46] J. Long, M. Zhong, H. Zhang, and P. Fan, "Superhydrophilicity to superhydrophobicity transition of picosecond laser microstructured aluminum in ambient air". Journal of Colloid and Interface Science, Vol. 441 (1) page 1-9, 2015 